\title{
Uncatalyzed Transfer Hydrogenation of Quinones and Related Systems: A Theoretical Mechanistic Study
}

\author{
Bun Chan* and Leo Radom* \\ School of Chemistry and Centre of Excellence in Free Radical Chemistry and \\ Biotechnology, University of Sydney, Sydney, NSW 2006, Australia
}

\section{Supporting Information}

(Tables S1-S7, Total 36 pages)

*Email: chan_b@chem.usyd.edu.au; radom@chem.usyd.edu.au 


\section{TABLE S1: GAUSSIAN Archive Entries for the B3-LYP/6-31+G(d,p) Optimized Geometries}

\section{Hydrogen donors and related compounds}

\section{Acridan (ACD)}

$1 \backslash 1 \backslash G I N C-A C 7 \backslash F O p t \backslash R B 3 L Y P \backslash 6-31+G(d, p) \backslash C 13 H 11 N 1 \backslash B U C 562 \backslash 02-J u n-2006 \backslash 0 \backslash \backslash \#$

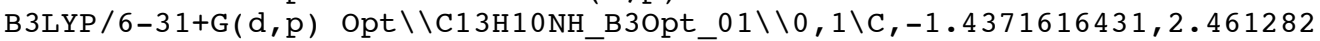
$2606,0.003793414 \backslash \mathrm{C},-2.833757241 \overline{8}, 2.45 \overline{6} 9661409,0.0031800889 \backslash \mathrm{C},-3.510180$ $9485,1.2330700164,0.0061390969 \backslash \mathrm{C},-2.7935144466,0.0392685289,0.00904540$ $28 \backslash \mathrm{C},-1.3878208235,0.0520869791,0.0091284842 \backslash \mathrm{N},-0.6818925367,-1.147285$ $2909,0.0123783274 \backslash \mathrm{C}, 0.7074183217,-1.2288168356,0.0067743764 \backslash \mathrm{C}, 1.336786$ $5281,-2.4857768838,0.0037346578 \backslash \mathrm{C}, 2.7260159363,-2.5793903152,-0.001911$ $9175 \backslash \mathrm{C}, 3.506951442,-1.4194137077,-0.0047395295 \backslash \mathrm{C}, 2.8741548616,-0.17442$ $62682,-0.0016490861 \backslash \mathrm{C}, 1.4812957066,-0.0526049446,0.004268333 \backslash \mathrm{C}, 0.82430$ $88025,1.3165344363,0.009237349 \backslash \mathrm{C},-0.693724428,1.2770862497,0.006947093$ $3 \backslash \mathrm{H},-0.9039932408,3.4097587023,0.001619013 \backslash \mathrm{H},-3.3843647901,3.392353603$ $3,0.0005327278 \backslash \mathrm{H},-4.595978236,1.2056883406,0.0057215314 \backslash \mathrm{H},-3.318541177$ $7,-0.9133737634,0.0109841547 \backslash \mathrm{H}, 0.7282749084,-3.3873695974,0.0057139891$ $\backslash \mathrm{H}, 3.1966359957,-3.5582719358,-0.0042161577 \backslash \mathrm{H}, 4.5904908805,-1.48305313$ $07,-0.0092418908 \backslash \mathrm{H}, 3.4753216864,0.7324675551,-0.0037086913 \backslash \mathrm{H}, 1.1707301$ $704,1.881402831,0.887085676 \backslash \mathrm{H},-1.2077946795,-2.0075103443,0.012379082 \backslash$ $\mathrm{H}, 1.1732679519,1.8890843736,-0.8624655251 \backslash \backslash$ Version=IA64L-G03RevD.01\St ate $=1-\mathrm{A} \backslash \mathrm{HF}=-556.8137866 \backslash \mathrm{RMSD}=4.294 \mathrm{e}-09 \backslash \mathrm{RMSF}=1.594 \mathrm{e}-05 \backslash \mathrm{Thermal}=0 . \backslash \mathrm{Dipol}$ $e=-0.1580458,-0.2586847,0.0004725 \backslash P G=C 01[X(C 13 \mathrm{H} 11 \mathrm{~N} 1)] \backslash \backslash @$

\section{Acridinium radical (ACD - $\mathbf{H} \bullet)$}

$1 \backslash 1 \backslash G I N C-A C 14 \backslash F O p t \backslash U B 3 L Y P \backslash 6-31+G(d, p) \backslash C 13 H 10 N 1(2) \backslash B U C 562 \backslash 02-J u n-2006 \backslash 0$

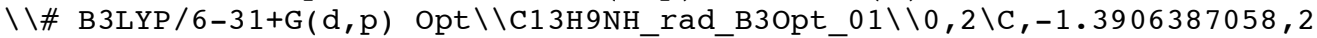
$.4742215581,0.0088647436 \backslash C,-2.7803 \overline{2} 513 \overline{6} 5,2.4 \overline{7} 46979138,0.01038265 \backslash \mathrm{C},-3$. $4839678655,1.2605540201,0.0104007786 \backslash \mathrm{C},-2.7888950674,0.0485952263,0.00$ $88859805 \backslash \mathrm{C},-1.3904161524,0.0410747153,0.0074826171 \backslash \mathrm{N},-0.6796266322,-1$. $151508561,0.0059328952 \backslash \mathrm{C}, 0.7056402154,-1.243748656,0.0042610633 \backslash \mathrm{C}, 1.34$ $7001117,-2.486464236,0.002646217 \backslash \mathrm{C}, 2.7424175244,-2.5557413825,0.001042$ $8547 \backslash \mathrm{C}, 3.5049737608,-1.3777242801,0.0010316477 \backslash \mathrm{C}, 2.8738553379,-0.13962$ $2523,0.002595842 \backslash \mathrm{C}, 1.4607253978,-0.0299436566,0.0042598249 \backslash \mathrm{C}, 0.7677091$ $179,1.2098186111,0.0058413622 \backslash \mathrm{C},-0.6515124928,1.2648165333,0.007394287$ $\backslash \mathrm{H},-0.8431629648,3.4129025041,0.0089083982 \backslash \mathrm{H},-3.3215147915,3.416002943$ $2,0.0116604106 \backslash \mathrm{H},-4.5694817389,1.2566038446,0.0116498627 \backslash \mathrm{H},-3.33135215$ $5,-0.8941055963,0.0088931367 \backslash \mathrm{H}, 0.7532451646,-3.397720594,0.0026685164 \backslash$ $\mathrm{H}, 3.2315258253,-3.5248282289,-0.0001849016 \backslash \mathrm{H}, 4.5893656539,-1.432798961$ $7,-0.0002151576 \backslash \mathrm{H}, 3.4618070304,0.7742447021,0.0025594752 \backslash \mathrm{H}, 1.335329258$ $6,2.1356375664,0.0058256319 \backslash \mathrm{H},-1.2075937012,-2.0128194622,0.0060098636$ $\backslash \backslash$ Version=IA64L-G03RevD.01 \State $=2-\mathrm{A} \backslash \mathrm{HF}=-556.1933101 \backslash \mathrm{S} 2=0.770886 \backslash \mathrm{S} 2-1=$ $0 . \backslash \mathrm{S} 2 \mathrm{~A}=0.750376 \backslash \mathrm{RMSD}=8.574 \mathrm{e}-09 \backslash \mathrm{RMSF}=9.350 \mathrm{e}-06 \backslash \mathrm{Thermal}=0 . \backslash \mathrm{Dipole}=-0.290$ $3031,-0.4737485,0.0001027 \backslash P G=C 01 \quad[X(C 13 H 10 N 1)] \backslash \backslash @$ 


\section{Acridinium cation (ACD - $\left.\mathrm{H}^{-}\right)$}

$1 \backslash 1 \backslash G I N C-A C 9 \backslash F O p t \backslash R B 3 L Y P \backslash 6-31+G(d, p) \backslash C 13 H 10 N 1(1+) \backslash B U C 562 \backslash 03-J u n-2006 \backslash 0$

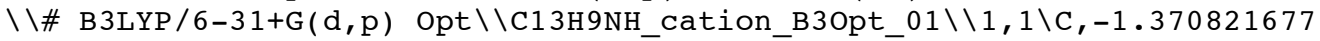
$8,2.4811756992,0.0088768979 \backslash \mathrm{C},-2.7 \overline{4} 385153 \overline{3} 7,2.4 \overline{7} 40102303,0.0104328282 \backslash$ $\mathrm{C},-3.452567417,1.2414689091,0.0103833638 \backslash \mathrm{C},-2.7913781063,0.0305961689$, $0.0088521037 \backslash \mathrm{C},-1.3804592832,0.0222151297,0.0074187542 \backslash \mathrm{N},-0.6787265945$ $,-1.1500763489,0.005844133 \backslash \mathrm{C}, 0.6843529957,-1.243374075,0.0042505522 \backslash \mathrm{C}$, $1.3321241472,-2.4967992579,0.0027123231 \backslash \mathrm{C}, 2.7111860978,-2.5364294895,0$ $.0011698921 \backslash \mathrm{C}, 3.4878313658,-1.3455285966,0.0011031636 \backslash \mathrm{C}, 2.8710992124,-$ $0.118795567,0.0025988185 \backslash \mathrm{C}, 1.4465217506,-0.0279609245,0.0042305181 \backslash \mathrm{C}, 0$ $.756961095,1.1923148348,0.0057987087 \backslash \mathrm{C},-0.6433628965,1.252987047,0.007$ $3690591 \backslash \mathrm{H},-0.8173103736,3.4149463291,0.0087296791 \backslash \mathrm{H},-3.2965689683,3.40$ $70994546,0.0117519644 \backslash \mathrm{H},-4.537852987,1.2532956016,0.011660199 \backslash \mathrm{H},-3.343$ $5157988,-0.9046377484,0.0088546735 \backslash \mathrm{H}, 0.7493222922,-3.4132386369,0.0027$ $74358 \backslash \mathrm{H}, 3.2142726948,-3.4981387052,-0.000007352 \backslash \mathrm{H}, 4.5701380657,-1.4145$ $601785,-0.0001412475 \backslash \mathrm{H}, 3.4520024912,0.7981836967,0.0025637351 \backslash \mathrm{H}, 1.3251$ $532167,2.119337695,0.0057373908 \backslash \mathrm{H},-1.2094417886,-2.0159472674,0.005833$ $4832 \backslash \backslash$ Version=IA64L-G03RevD.01 \State $=1-A \backslash H F=-555.9926737 \backslash$ RMSD $=7.414 \mathrm{e}-0$ $9 \backslash \mathrm{RMSF}=7.554 \mathrm{e}-06 \backslash \mathrm{Thermal}=0 . \backslash \mathrm{Dipole}=-0.2334093,-0.3808943,0.0000191 \backslash \mathrm{PG}=$ $\mathrm{C} 01[\mathrm{X}(\mathrm{C} 13 \mathrm{H} 10 \mathrm{~N} 1)] \backslash \backslash @$

\section{Acridine (ACD - $\left.\mathbf{H}_{2}\right)$}

$1 \backslash 1 \backslash G I N C-A C 16 \backslash F O p t \backslash R B 3 L Y P \backslash 6-31+G(d, p) \backslash C 13 H 9 N 1 \backslash B U C 562 \backslash 02-J u n-2006 \backslash 0 \backslash \backslash \#$

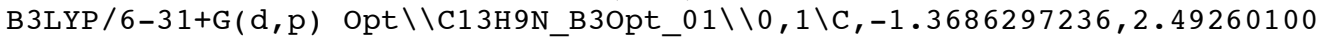
$12,0.0089926661 \backslash \mathrm{C},-2.73989138 \overline{6} 6,2.4 \overline{6} 91335511,0.0103204405 \backslash \mathrm{C},-3.4410076$ $603,1.2250119042,0.0098207578 \backslash \mathrm{C},-2.7596970271,0.034462491,0.0078564015$ $\backslash \mathrm{C},-1.3285553956,0.0131660618,0.0062063961 \backslash \mathrm{N},-0.7020210315,-1.17594432$ $18,0.0046442701 \backslash \mathrm{C}, 0.6418941048,-1.197572266,0.0034580637 \backslash \mathrm{C}, 1.307663556$ $3,-2.4645822556,0.00174597 \backslash \mathrm{C}, 2.6775992146,-2.5343143462,0.0004227387 \backslash \mathrm{C}$ , $3.4705949596,-1.346641616,0.0009500421 \backslash \mathrm{C}, 2.8718470327,-0.1127814441,0$ $.0027235017 \backslash \mathrm{C}, 1.4466387684,0.0043044527,0.0039806929 \backslash \mathrm{C}, 0.7779341483,1$. $232747977,0.0058360569 \backslash \mathrm{C},-0.6201586445,1.2741885935,0.0070414095 \backslash \mathrm{H},-0$. $8292514861,3.4365361034,0.0091876424 \backslash \mathrm{H},-3.3014938518,3.3986115747,0.01$ $19832029 \backslash \mathrm{H},-4.5272377242,1.2285312017,0.010646245 \backslash \mathrm{H},-3.2747888833,-0.9$ $207116293,0.0072530764 \backslash \mathrm{H}, 0.6884160817,-3.3557647749,0.0013751791 \backslash \mathrm{H}, 3.1$ $715895905,-3.5017223066,-0.0010449203 \backslash \mathrm{H}, 4.5535468944,-1.4275782067,-0$. $0001650774 \backslash \mathrm{H}, 3.4701779169,0.7949288901,0.0031072144 \backslash \mathrm{H}, 1.3476975467,2.1$ $60010365,0.0063700299 \backslash \backslash$ Version=IA64L-G03RevD.01 \State $=1-A \backslash H F=-555.6038$ $166 \backslash \mathrm{RMSD}=5.714 \mathrm{e}-09 \backslash \mathrm{RMSF}=1.836 \mathrm{e}-05 \backslash \mathrm{Thermal}=0 . \backslash \mathrm{Dipole}=0.416484,0.6777091$ , $0.000198 \backslash \mathrm{PG}=\mathrm{C} 01[\mathrm{X}(\mathrm{C} 13 \mathrm{H} 9 \mathrm{~N} 1)] \backslash \backslash @$

\section{1,4-Cyclohexadiene (CHD)}

$1 \backslash 1 \backslash G I N C-A C 17 \backslash F O p t \backslash R B 3 L Y P \backslash 6-31+G(d, p) \backslash C 6 H 8 \backslash B U C 562 \backslash 22-S e p-2005 \backslash 0 \backslash \backslash \# B 3 L$

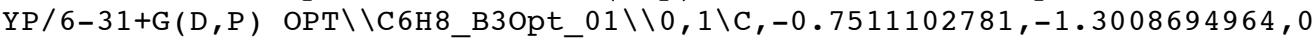
$.0006456892 \backslash \mathrm{C}, 0.75338437 \overline{3} 3,-1 . \overline{2} 073047916,0.0010555122 \backslash \mathrm{C}, 1.4222462007$,$0.0488814674,0.0004540355 \backslash \mathrm{C}, 0.7511070105,1.3008722531,-0.0007201289 \backslash \mathrm{C}$, $-0.7533824921,1.2073045333,-0.0010121542 \backslash \mathrm{C},-1.4222448375,0.0488793343$, $-0.00040899 \backslash \mathrm{H},-1.0888905723,-1.8848734769,0.8720194982 \backslash \mathrm{H}, 1.3049749899$, $-2.1458484337,0.0018978202 \backslash \mathrm{H}, 2.5108437818,-0.0573631936,0.0008138789 \backslash \mathrm{H}$ , $1.0888269819,1.8847591686,-0.8721973571 \backslash \mathrm{H},-1.3049790278,2.1458450164$, $-0.0017637297 \backslash \mathrm{H},-2.5108426792,0.0573680668,-0.0006773882 \backslash \mathrm{H}, 1.088417422$ $5,1.886076633,0.8700288995 \backslash \mathrm{H},-1.088350757,-1.8859659725,-0.8702054051 \backslash$ $\backslash$ Version $=I A 64 \mathrm{~L}-\mathrm{G} 03 \mathrm{RevC} .02 \backslash \mathrm{State}=1-\mathrm{A} \backslash \mathrm{HF}=-233.439841 \backslash \mathrm{RMSD}=6.823 \mathrm{e}-09 \backslash \mathrm{RMSF}$ $=3.769 e-05 \backslash$ Dipole $=0.0000004,-0.0000011,-0.0000246 \backslash P G=C 01[X(C 6 H 8)] \backslash \backslash @$ 


\section{Cyclohexadienyl radical (CHD - H•)}

$1 \backslash 1 \backslash G I N C-A C 17 \backslash F O p t \backslash U B 3 L Y P \backslash 6-31+G(d, p) \backslash C 6 H 7(2) \backslash B U C 562 \backslash 22-S e p-2005 \backslash 0 \backslash \backslash \#$

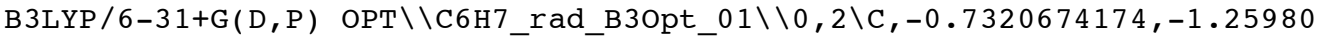
$96491,0.06075197 \backslash C, 0.688613 \overline{3} 164,-1.25 \overline{8} 1606623,0.0322199496 \backslash C, 1.4009283$ $861,-0.0908927465,-0.0249584607 \backslash \mathrm{C}, 0.7284738616,1.2536475869,-0.0604881$ $648 \backslash \mathrm{C},-0.7726784791,1.1720742332,-0.0275460879 \backslash \mathrm{C},-1.434093085,-0.02477$ $28107,0.0296992591 \backslash \mathrm{H},-1.2766949131,-2.1970315019,0.1067106146 \backslash \mathrm{H}, 1.2185$ $535224,-2.2073753094,0.0563711751 \backslash \mathrm{H}, 2.4875548823,-0.1135264729,-0.0455$ $898038 \backslash \mathrm{H}, 1.0564638511,1.8162494688,-0.9550169097 \backslash \mathrm{H},-1.3304486812,2.104$ $8463964,-0.0502315981 \backslash \mathrm{H},-2.5212223892,-0.0344191624,0.0519085402 \backslash \mathrm{H}, 1.0$ $907342316,1.878740873,0.7777771905 \backslash \backslash$ Version=IA64L-G03RevC.02 $\backslash$ State $=2-A$ $\backslash \mathrm{HF}=-232.8142807 \backslash \mathrm{S} 2=0.787741 \backslash \mathrm{S} 2-1=0 . \backslash \mathrm{S} 2 \mathrm{~A}=0.750802 \backslash \mathrm{RMSD}=5.616 \mathrm{e}-09 \backslash \mathrm{RMSF}=$ $2.507 e-05 \backslash \mathrm{Dipole}=0.1071398,0.1843462,-0.0083154 \backslash \mathrm{PG}=\mathrm{C} 01 \quad[\mathrm{X}(\mathrm{C} 6 \mathrm{H} 7)] \backslash \backslash @$

\section{Cyclohexadienyl cation $\left(\mathrm{CHD}-\mathrm{H}^{-}\right)$}

$1 \backslash 1 \backslash G I N C-A C 6 \backslash F O p t \backslash R B 3 L Y P \backslash 6-31+G(d, p) \backslash C 6 H 7(1+) \backslash B U C 562 \backslash 22-$ Sep-2005 $10 \backslash \backslash \#$ B3LYP / 6-31+G(D,P) OPT \\C6H7 cation B3Opt $01 \backslash \backslash 1,1 \backslash C,-0.7113513842,-1.22$ $42103559,0.0586846669 \backslash \mathrm{C}, 0.7 \overline{0} 118830 \overline{9} 5,-1 . \overline{2} 651837129,0.0320458676 \backslash \mathrm{C}, 1.40$ $21099265,-0.0860492913,-0.0248704748 \backslash \mathrm{C}, 0.702355745,1.2087249003,-0.057$ $9184834 \backslash C,-0.7690834975,1.1754254492,-0.0274940854 \backslash C,-1.4464659221,-0$. $0173920595,0.0294486736 \backslash \mathrm{H},-1.2573686652,-2.1638701827,0.1036911638 \backslash \mathrm{H}, 1$ $.2133572468,-2.221017405,0.0565510221 \backslash \mathrm{H}, 2.4887396558,-0.0831326527,-0$. $0467020392 \backslash \mathrm{H}, 1.0423060339,1.7920748942,-0.9376349185 \backslash \mathrm{H},-1.3046257565,2$ $.1208782227,-0.0512943421 \backslash \mathrm{H},-2.5305410566,-0.045745842,0.0520104263 \backslash \mathrm{H}$, $1.0756134792,1.8529233863,0.7640017005 \backslash \backslash$ Version=IA64L-G03RevC.02 $\backslash$ State $=1-\mathrm{A} \backslash \mathrm{HF}=-232.5701854 \backslash \mathrm{RMSD}=4.938 \mathrm{e}-09 \backslash \mathrm{RMSF}=1.048 \mathrm{e}-05 \backslash \mathrm{Dipole}=0.1764384,0$. $3037021,-0.0143302 \backslash P G=C 01[X(\mathrm{C} 6 \mathrm{H} 7)] \backslash \backslash @$

\section{Benzene (CHD - $\left.\mathrm{H}_{2}\right)$}

$1 \backslash 1 \backslash$ GINC-AC6 FOpt \RB3LYP $\backslash 6-31+G(d, p) \backslash C 6 H 6 \backslash B U C 562 \backslash 22-S e p-2005 \backslash 0 \backslash \backslash \#$ B3LY

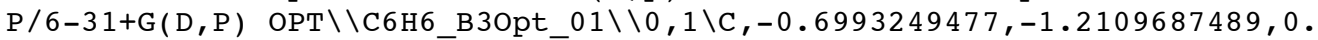
$0005559411 \backslash \mathrm{C}, 0.69916571 \overline{1},-1.2 \overline{1} 0952577,0.0010711352 \backslash \mathrm{C}, 1.3984353676,-0.0$ $001127674,0.0003857516 \backslash \mathrm{C}, 0.699223132,1.2110280675,-0.0005453064 \backslash \mathrm{C},-0.6$ $990637916,1.2110087221,-0.0009354481 \backslash \mathrm{C},-1.3984368253,-0.0000012659,-0$. $0005070091 \backslash \mathrm{H},-1.2423127045,-2.1517104926,0.000918112 \backslash \mathrm{H}, 1.242091201,-2$. $1517548326,0.001855292 \backslash \mathrm{H}, 2.4846279349,-0.0000885995,0.0006555783 \backslash \mathrm{H}, 1.2$ $424450634,2.1516272176,-0.0009687838 \backslash \mathrm{H},-1.2422067617,2.1516783025,-0.0$ $017636753 \backslash \mathrm{H},-2.4846366098,0.0002398226,-0.0008469094 \backslash \backslash$ Vers ion=IA64L-G0 3 RevC.02 $\backslash$ State $=1-A \backslash H F=-232.268418 \backslash \mathrm{RMSD}=2.425 \mathrm{e}-09 \backslash \mathrm{RMSF}=1.062 \mathrm{e}-04 \backslash \mathrm{Dipole}$ $=0.0000035,0.0000027,-0.0000466 \backslash P G=C 01 \quad[\mathrm{X}(\mathrm{C} 6 \mathrm{H} 6)] \backslash \backslash @$

\section{9,10-Dihydroanthracene (DHA)}

$1 \backslash 1 \backslash$ GINC-AC $11 \backslash F O p t \backslash R B 3 L Y P \backslash 6-31+G(d, p) \backslash C 14$ H $12 \backslash B U C 562 \backslash 23-A u g-2006 \backslash 0 \backslash \backslash \# B$

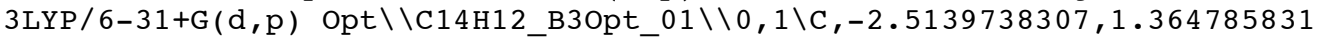
$5,-0.0010918405 \backslash C,-3.7248218 \overline{2} 9,0.6 \overline{7} 81995941,0.0010552586 \backslash C,-3.72253129$ $78,-0.721750046,0.0010700111 \backslash \mathrm{C},-2.5095660044,-1.4045202106,-0.00105437$ $31 \backslash \mathrm{C},-1.2827886467,-0.7185951591,-0.0032810563 \backslash \mathrm{C}, 0.0017396511,-1.51932$ $65469,-0.005831555 \backslash \mathrm{C}, 1.283835164,-0.7147870212,-0.00320611 \backslash \mathrm{C}, 2.5127163$ $057,-1.3969343733,-0.000882165 \backslash C, 3.7236079058,-0.7104682429,0.00123511$ $84 \backslash C, 3.7216247543,0.6894802246,0.0011415141 \backslash C, 2.508706514,1.3723818927$ $,-0.0010943607 \backslash \mathrm{C}, 1.2819917689,0.6865014259,-0.0033155822 \backslash \mathrm{C},-0.00280060$ $43,1.4878804656,-0.0061676385 \backslash C,-1.2851420727,0.6826959871,-0.00332955$ $18 \backslash \mathrm{H},-2.5126016559,2.4528560665,-0.0010321138 \backslash \mathrm{H},-4.6625415382,1.226432$ $1859,0.0027851728 \backslash \mathrm{H},-4.6584117904,-1.2731315184,0.0028062071 \backslash \mathrm{H},-2.5050$ $140757,-2.4925923693,-0.0009385566 \backslash \mathrm{H}, 2.5114144994,-2.4850122398,-0.000$ $7129293 \backslash \mathrm{H}, 4.661184669,-1.2589617669,0.0030332391 \backslash \mathrm{H}, 4.6576559434,1.2405$ $89938,0.0028782221 \backslash \mathrm{H}, 2.5040809827,2.4604427866,-0.0010932213 \backslash \mathrm{H},-0.0038$ $986795,2.1588672635,0.8646828095 \backslash \mathrm{H}, 0.0028350297,-2.1908909725,0.864797$ $2796 \backslash \mathrm{H},-0.0038094847,2.1526386537,-0.8818849223 \backslash \mathrm{H}, 0.0028053219,-2.1854$ $078488,-0.8807268558 \backslash \backslash$ Version=IA64L-G03RevD. $01 \backslash$ State $=1-A \backslash H F=-540.76880$ $72 \backslash \mathrm{RMSD}=6.288 \mathrm{e}-09 \backslash \mathrm{RMSF}=6.756 \mathrm{e}-05 \backslash \mathrm{Thermal}=0 . \backslash \mathrm{Dipole}=-0.0000012,-0.00028$ $24,-0.0014187 \backslash \mathrm{PG}=\mathrm{C} 01[\mathrm{X}(\mathrm{C} 14 \mathrm{H} 12)] \backslash \backslash @$ 


\section{Hydroanthracenyl radical (DHA - H•)}

$1 \backslash 1 \backslash G I N C-A C 7 \backslash F O p t \backslash U B 3 L Y P \backslash 6-31+G(d, p) \backslash C 14 H 11(2) \backslash B U C 562 \backslash 23-A u g-2006 \backslash 0 \backslash \backslash \#$

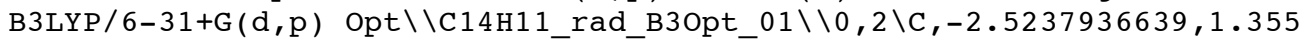
$5625628,-0.0010852351 \backslash C,-3.724 \overline{8} 065 \overline{5} 48,0 . \overline{6} 399873815,0.0004838559 \backslash C,-3.6$ $979794453,-0.7631948853,0.0006357736 \backslash C,-2.4820362347,-1.4305630851,-0$. $0004619751 \backslash \mathrm{C},-1.2534844489,-0.7191077824,-0.0018651424 \backslash \mathrm{C},-0.0026294631$ $,-1.4037193015,-0.0022588934 \backslash C, 1.2470855945,-0.7171133988,-0.001686274$ $5 \backslash \mathrm{C}, 2.4767707687,-1.426589994,0.0000578257 \backslash \mathrm{C}, 3.691620844,-0.7572469255$ $, 0.0013938645 \backslash \mathrm{C}, 3.7161128259,0.646015537,0.0011002617 \backslash \mathrm{C}, 2.5139723868,1$ $.3595733019,-0.0007345955 \backslash \mathrm{C}, 1.2802201961,0.7062305239,-0.0022422207 \backslash \mathrm{C}$, $-0.0050477343,1.5077508516,-0.0054208348 \backslash \mathrm{C},-1.2890599299,0.7041537623$, $-0.0023288439 \backslash \mathrm{H},-2.549115074,2.443368359,-0.0013293293 \backslash \mathrm{H},-4.6729093008$ $, 1.1693074138,0.0015165078 \backslash \mathrm{H},-4.6270643029,-1.3259967671,0.0017089089 \backslash$ $\mathrm{H},-2.4552532305,-2.5173181162,-0.0001599743 \backslash \mathrm{H}, 2.4516650087,-2.51338012$ $69,0.0004647825 \backslash \mathrm{H}, 4.6216369745,-1.3184953017,0.0027466679 \backslash \mathrm{H}, 4.66330076$ $94,1.1769756346,0.0022769072 \backslash \mathrm{H}, 2.5376125662,2.4474465699,-0.0010206511$ $\backslash \mathrm{H},-0.0059962634,2.1835669391,0.8634673474 \backslash \mathrm{H},-0.0018205793,-2.49057839$ $63,-0.0017943093 \backslash \mathrm{H},-0.005829709,2.1759332434,-0.8803584237 \backslash \backslash$ Version=IA $64 \mathrm{~L}-\mathrm{G} 03 \mathrm{RevD} .01 \backslash \mathrm{State}=2-\mathrm{A} \backslash \mathrm{HF}=-540.1406212 \backslash \mathrm{S} 2=0.782428 \backslash \mathrm{S} 2-1=0 . \backslash \mathrm{S} 2 \mathrm{~A}=0.750$ $951 \backslash \mathrm{RMSD}=8.804 \mathrm{e}-09 \backslash \mathrm{RMSF}=1.033 \mathrm{e}-04 \backslash \mathrm{Thermal}=0 . \backslash \mathrm{Dipole}=-0.0001207,0.18691$ $79,-0.0005935 \backslash \mathrm{PG}=\mathrm{C} 01 \quad[\mathrm{X}(\mathrm{C} 14 \mathrm{H} 11)] \backslash \backslash @$

\section{Hydroanthracenyl cation (DHA - $\mathrm{H}^{-}$)}

$1 \backslash 1 \backslash G I N C-A C 4 \backslash F O p t \backslash R B 3 L Y P \backslash 6-31+G(d, p) \backslash C 14 H 11(1+) \backslash B U C 562 \backslash 23-A u g-2006 \backslash 0 \backslash \backslash$

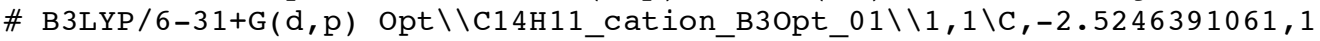
$.3677952805,-0.0009923324 \backslash \mathrm{C},-3 . \overline{7} 033650 \overline{8} 21,0 . \overline{6} 169661391,0.0003132682 \backslash \mathrm{C}$, $-3.6763502961,-0.7913079587,0.0003046722 \backslash C,-2.4605964793,-1.4449319698$ $,-0.0006968116 \backslash \mathrm{C},-1.2484805654,-0.6968492193,-0.0018187142 \backslash \mathrm{C},-0.002677$ $5557,-1.3549055094,-0.0016762226 \backslash \mathrm{C}, 1.2420549108,-0.6948453865,-0.00143$ $09393 \backslash C, 2.4553600804,-1.4409762708,0.0001282797 \backslash C, 3.6700497157,-0.7853$ $630849,0.0011890604 \backslash \mathrm{C}, 3.6946864696,0.6229702551,0.0007650942 \backslash \mathrm{C}, 2.51476$ $48797,1.3718290384,-0.0008834093 \backslash C, 1.2754084741,0.7344802177,-0.002067$ $1151 \backslash \mathrm{C},-0.0050849284,1.5194355455,-0.004870483 \backslash \mathrm{C},-1.2843355487,0.73236$ $39172,-0.0021342614 \backslash \mathrm{H},-2.5790513671,2.4525912045,-0.0010405792 \backslash \mathrm{H},-4.65$ $96999472,1.1313223384,0.0013124545 \backslash \mathrm{H},-4.604659168,-1.3520919658,0.0011$ $956236 \backslash \mathrm{H},-2.4132799645,-2.5299114824,-0.0005280998 \backslash \mathrm{H}, 2.4098046036,-2.5$ $260256181,0.0006340062 \backslash \mathrm{H}, 4.5992748005,-1.3446216701,0.0024218688 \backslash \mathrm{H}, 4.6$ $501102115,1.139021994,0.0017696934 \backslash \mathrm{H}, 2.567522172,2.4567293775,-0.00119$ $07923 \backslash \mathrm{H},-0.0059759552,2.2004819718,0.8599818116 \backslash \mathrm{H},-0.0017989826,-2.443$ $9102855,-0.0011852211 \backslash \mathrm{H},-0.0058693714,2.1923211415,-0.8763948517 \backslash \backslash$ Vers ion $=I A 64 \mathrm{~L}-\mathrm{G} 03 \mathrm{RevD} .01 \backslash$ State $=1-\mathrm{A} \backslash \mathrm{HF}=-539.9162638 \backslash \mathrm{RMSD}=7.188 \mathrm{e}-09 \backslash \mathrm{RMSF}=9.5$ $97 \mathrm{e}-05 \backslash \mathrm{Thermal}=0 . \backslash \mathrm{Dipole}=0.0000686,0.0020972,-0.0007912 \backslash \mathrm{PG}=\mathrm{C} 01 \quad[\mathrm{X}(\mathrm{C} 14 \mathrm{H}$ $11)] \backslash \backslash @$

\section{Anthracene (DHA - $\mathbf{H}_{2}$ )}

$1 \backslash 1 \backslash G I N C-A C 4 \backslash F O p t \backslash R B 3 L Y P \backslash 6-31+G(d, p) \backslash C 14 H 10 \backslash B U C 562 \backslash 23-A u g-2006 \backslash 0 \backslash \backslash \#$ B3

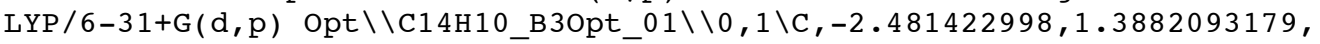
$0.0000148752 \backslash \mathrm{C},-3.664227822,0.6937829894,0.0000186024 \backslash \mathrm{C},-3.6640986782$, $-0.733871919,0.0000218703 \backslash \mathrm{C},-2.4814381486,-1.4283299813,0.0000202381 \backslash \mathrm{C}$ $,-1.2247087641,-0.7428949885,0.0000165187 \backslash \mathrm{C}, 0.0000053223,-1.4250667549$ $, 0.0000147852 \backslash \mathrm{C}, 1.2247177325,-0.7428934935,0.0000120356 \backslash \mathrm{C}, 2.4814495746$ $,-1.4283236165,0.0000122159 \backslash \mathrm{C}, 3.664108057,-0.7338622147,0.0000054555 \backslash \mathrm{C}$ , 3.6642329059,0.6937931518,0.000002124\C,2.4814264001,1.3882154082,0.0 $000034129 \backslash \mathrm{C}, 1.224707172,0.7027523238,0.0000061825 \backslash \mathrm{C}, 0.0000021321,1.384$ $7463527,0.0000068347 \backslash \mathrm{C},-1.2247032749,0.702749679,0.000012753 \backslash \mathrm{H},-2.4801$ $627479,2.4752444334,0.0000090735 \backslash \mathrm{H},-4.6100984584,1.2276643219,0.000022$ $1469 \backslash \mathrm{H},-4.6100293454,-1.267634823,0.000024046 \backslash \mathrm{H},-2.4794306835,-2.51538$ $62329,0.0000152953 \backslash \mathrm{H}, 2.4794464349,-2.5153799373,0.0000141558 \backslash \mathrm{H}, 4.61003$ $99559,-1.267622767,0.0000039865 \backslash \mathrm{H}, 4.6101020073,1.2276772635,-0.0000019$ $437 \backslash \mathrm{H}, 2.4801617817,2.4752506174,-0.0000000991 \backslash \mathrm{H}, 0.0000006434,2.4726551$ $853,0.0000034938 \backslash \mathrm{H}, 0.000004808,-2.5129253156,0.0000199411 \backslash \backslash$ Version=IA6 $4 \mathrm{~L}-\mathrm{G} 03$ RevD. $01 \backslash \mathrm{State}=1-\mathrm{A} \backslash \mathrm{HF}=-539.5643852 \backslash \mathrm{RMSD}=9.238 \mathrm{e}-09 \backslash \mathrm{RMSF}=6.724 \mathrm{e}-05 \backslash$ Thermal $=0 . \backslash$ Dipole $=-0.0000006,0.0001517,-0.0000026 \backslash \mathrm{PG}=\mathrm{C} 01[\mathrm{X}(\mathrm{C} 14 \mathrm{H} 10)] \backslash \backslash$ a 


\section{1,4-Dihydropyridine (DHP)}

$1 \backslash 1 \backslash G I N C-A C 6 \backslash F O p t \backslash R B 3 L Y P \backslash 6-31+G(d, p) \backslash C 5 H 7 N 1 \backslash B U C 562 \backslash 22-S e p-2005 \backslash 0 \backslash \backslash \#$ B3

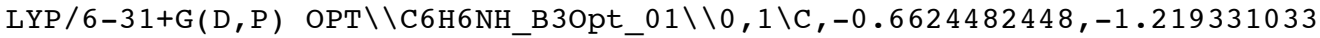
$8,-0.0332592612 \backslash \mathrm{C}, 0.6802239314,-1.2219531625,-0.045017903 \backslash \mathrm{C}, 1.49927895$ $22,0.0470429739,0.0843877529 \backslash \mathrm{C}, 0.6020577806,1.2624128675,-0.0415563744$ $\backslash \mathrm{C},-0.7377923684,1.1753740099,-0.029927717 \backslash \mathrm{N},-1.4101384354,-0.04449771$ $79,0.0969654519 \backslash \mathrm{H}, 2.0416148182,0.062778552,1.0462988468 \backslash \mathrm{H},-1.245390780$ $4,-2.1314622994,-0.1079031985 \backslash \mathrm{H}, 1.1967550268,-2.1728253488,-0.13305798$ $27 \backslash \mathrm{H}, 1.0578124139,2.2441031747,-0.1269166168 \backslash \mathrm{H},-1.3769211517,2.0492643$ $801,-0.1020765638 \backslash \mathrm{H},-2.379088272,-0.0745988141,-0.179501966 \backslash \mathrm{H}, 2.288266$ $6865,0.0729504504,-0.6833596665 \backslash \backslash$ Version=IA64L-G03RevC .02 $\backslash$ State $=1-A \backslash H F$ $=-249.4808389 \backslash \mathrm{RMSD}=2.262 \mathrm{e}-09 \backslash \mathrm{RMSF}=4.255 \mathrm{e}-06 \backslash \mathrm{Dipole}=-0.5322299,-0.01649$ $34,-0.1759258 \backslash \mathrm{PG}=\mathrm{C} 01 \quad[\mathrm{X}(\mathrm{C} 5 \mathrm{H} 7 \mathrm{~N} 1)] \backslash \backslash @$

\section{Pyridinium radical (DHP - H•)}

$1 \backslash 1 \backslash G I N C-A C 17 \backslash F O p t \backslash U B 3 L Y P \backslash 6-31+G(d, p) \backslash C 5 H 6 N 1(2) \backslash B U C 562 \backslash 22-S e p-2005 \backslash 0 \backslash \backslash$ \# B3LYP/6-31+G(D,P) OPT \\C5H5NH rad B3Opt $01 \backslash \backslash 0,2 \backslash C,-0.6170792993,-1.2$ $334910741,-0.0392365179 \backslash \mathrm{C}, 0.748 \overline{4} 090805,-1.1941719958,0.0237883588 \backslash \mathrm{C}, 1$. $4472779103,0.0445666847,0.0735188933 \backslash \mathrm{C}, 0.6732890422,1.2379783753,0.027$ $7500115 \backslash \mathrm{C},-0.6920248526,1.1932144733,-0.0352710666 \backslash \mathrm{N},-1.3544817529,-0$. $0418056582,-0.0156547049 \backslash \mathrm{H}, 2.5286001065,0.0779263981,0.1188279743 \backslash \mathrm{H},-1$ $.1929354572,-2.1494532387,-0.0767432032 \backslash \mathrm{H}, 1.2905542721,-2.1347487823,0$ $.0317229087 \backslash \mathrm{H}, 1.1563949058,2.2101714785,0.0388141287 \backslash \mathrm{H},-1.3233329322,2$ $.0719925457,-0.0698656455 \backslash \mathrm{H},-2.3371399117,-0.071827575,-0.2364713036 \backslash \backslash$ Version=IA6 4L-G03RevC.02 \State $=2-A \backslash H F=-248.8613996 \backslash \mathrm{S} 2=0.769686 \backslash \mathrm{S} 2-1=0$. $\backslash \mathrm{S} 2 \mathrm{~A}=0.750256 \backslash \mathrm{RMSD}=7.442 \mathrm{e}-09 \backslash \mathrm{RMSF}=9.697 \mathrm{e}-06 \backslash \mathrm{Dipole}=-0.7542045,-0.02305$ $37,-0.1455007 \backslash \mathrm{PG}=\mathrm{C} 01 \quad[\mathrm{X}(\mathrm{C} 5 \mathrm{H} 6 \mathrm{~N} 1)] \backslash \backslash @$

\section{Pyridinium cation (DHP $\left.-\mathrm{H}^{-}\right)$}

$1 \backslash 1 \backslash G I N C-A C 26 \backslash F O p t \backslash R B 3 L Y P \backslash 6-31+G(d, p) \backslash C 5 H 6 N 1(1+) \backslash B U C 562 \backslash 22-$ Sep-2005 $0 \backslash$

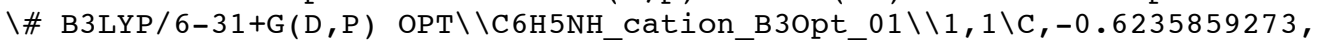
$-1.2142974371,0.0001199244 \backslash \mathrm{C}, 0.7 \overline{6} 103047 \overline{3} 7,-1 . \overline{1} 853028721,0.0002152155 \backslash \mathrm{C}$ $, 1.4158386226,0.0516777178,0.0000978828 \backslash \mathrm{C}, 0.6725956424,1.237630089,-0$. $0001172674 \backslash \mathrm{C},-0.7104493217,1.1656060646,-0.0002130173 \backslash \mathrm{N},-1.3092866291$, $-0.0477892037,-0.0000898938 \backslash \mathrm{H},-1.2091041811,-2.1259309077,0.0002014243$ $\backslash \mathrm{H}, 1.312901087,-2.118180231,0.0003838033 \backslash \mathrm{H}, 1.1549871258,2.208258994,-0$ $.0002116399 \backslash \mathrm{H},-1.3608716068,2.0321270901,-0.0003773416 \backslash \mathrm{H},-2.326036906$, $-0.0848998019,-0.0001569839 \backslash \mathrm{H}, 2.500553947,0.0912679092,0.0001735667 \backslash \backslash \mathrm{V}$ ersion $=I A 64 \mathrm{~L}-\mathrm{G} 03 \mathrm{RevC} .02 \backslash$ State $=1-\mathrm{A} \backslash \mathrm{HF}=-248.6717933 \backslash \mathrm{RMSD}=8.900 \mathrm{e}-09 \backslash \mathrm{RMSF}=$ $8.289 e-05 \backslash \mathrm{Dipole}=-0.734592,-0.0268129,-0.0000475 \backslash \mathrm{PG}=\mathrm{C} 01 \quad[\mathrm{X}(\mathrm{C} 5 \mathrm{H} 6 \mathrm{~N} 1)] \backslash \backslash @$

\section{Pyridine (DHP $\left.-\mathrm{H}_{2}\right)$}

$1 \backslash 1 \backslash G I N C-A C 40 \backslash F O p t \backslash R B 3 L Y P \backslash 6-31+G(d, p) \backslash C 5 H 5 N 1 \backslash B U C 562 \backslash 22-S e p-2005 \backslash 0 \backslash \backslash \# B$

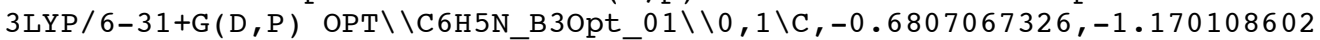
$6,0.0001156001 \backslash \mathrm{C}, 0.71686672 \overline{6} 1,-1 . \overline{1} 744084697,0.0002184301 \backslash \mathrm{C}, 1.385869245$ $, 0.0505795763,0.0001098932 \backslash \mathrm{C}, 0.6293637566,1.2235435047,-0.0001135041 \backslash \mathrm{C}$ $,-0.764176211,1.1173750913,-0.0002340975 \backslash N,-1.4200061392,-0.0518271618$ $,-0.000110371 \backslash \mathrm{H},-1.2339749162,-2.107036775,0.000185011 \backslash \mathrm{H}, 1.2604585294$, $-2.1141779716,0.0003882196 \backslash \mathrm{H}, 1.1029783156,2.2004534189,-0.0001995692 \backslash \mathrm{H}$ $,-1.384275,2.0114735167,-0.0003757312 \backslash \mathrm{H}, 2.4715553408,0.0901913437,0.00$ $0196736 \backslash \backslash$ Version=IA64L-G03RevC.02 \State $=1-A \backslash H F=-248.3033683 \backslash \mathrm{RMSD}=4.696$ $\mathrm{e}-09 \backslash \mathrm{RMSF}=9.439 \mathrm{e}-05 \backslash \mathrm{Dipole}=0.9342653,0.0340982,0.0000773 \backslash \mathrm{PG}=\mathrm{C} 01 \quad[\mathrm{X}(\mathrm{C} 5 \mathrm{H}$ $5 \mathrm{~N} 1)] \backslash \backslash \mathrm{Q}$ 


\section{Hydrogen acceptors and related compounds}

\section{$\alpha$-Methylstyrene (AMS)}

$1 \backslash 1 \backslash G I N C-A C 1 \backslash F O p t \backslash R B 3 L Y P \backslash 6-31+G(d, p) \backslash C 9 H 10 \backslash B U C 562 \backslash 08-J u n-2006 \backslash 0 \backslash \backslash \#$ B 3L $\mathrm{YP} / 6-31+\mathrm{G}(\mathrm{d}, \mathrm{p})$ Opt $\backslash \backslash \mathrm{PhMeC} 2 \mathrm{H} 2 \_\mathrm{B} 3 \mathrm{Opt} \backslash \backslash 0,1 \backslash \mathrm{C},-2.6179354013,-0.0733663762$, $-0.0322651084 \backslash C,-1.859759602 \overline{1},-1.231577349,-0.2153436786 \backslash C,-0.46442278$ $53,-1.1709169648,-0.1988877625 \backslash \mathrm{C}, 0.2095554335,0.0501781949,-0.01317828$ $89 \backslash \mathrm{C},-0.571726695,1.2047930923,0.1864882658 \backslash \mathrm{C},-1.9648418003,1.14604056$ $63,0.1725365688 \backslash \mathrm{H},-3.7029932989,-0.1205447799,-0.0387960197 \backslash \mathrm{H},-2.35313$ $95933,-2.1869061263,-0.3712793155 \backslash \mathrm{H}, 0.102042594,-2.0842268594,-0.34861$ $72139 \backslash \mathrm{H},-0.0803261404,2.1533382852,0.3788409207 \backslash \mathrm{H},-2.5418443428,2.0521$ $808951,0.3351607966 \backslash \mathrm{C}, 1.6982660097,0.1176189505,-0.0162029287 \backslash \mathrm{C}, 2.4590$ $83605,-1.1020104922,0.4567488836 \backslash \mathrm{C}, 2.3552393666,1.2143793881,-0.432764$ $455 \backslash \mathrm{H}, 3.4400559412,1.260017539,-0.4110522466 \backslash \mathrm{H}, 1.8392515005,2.08528852$ $55,-0.8240389163 \backslash \mathrm{H}, 3.5298353134,-0.8913443979,0.5181435936 \backslash \mathrm{H}, 2.1117013$ $809,-1.4313255901,1.4429785845 \backslash \mathrm{H}, 2.3289255145,-1.948973501,-0.22784267$ 95 \Version=IA64L-G03RevD.01 \State $=1-A \backslash H F=-348.9928847 \backslash R M S D=6.464 e-09 \backslash$ RMSF $=1.050 \mathrm{e}-05 \backslash \mathrm{Thermal}=0 . \backslash \mathrm{Dipole}=0.0024436,-0.12839,0.0515702 \backslash \mathrm{PG}=\mathrm{C} 01$ [ $\mathrm{X}(\mathrm{C} 9 \mathrm{H} 10)] \backslash \backslash \mathrm{Q}$

\section{2-Phenylpropanyl radical (AMS + $\mathrm{H} \bullet$ )}

$1 \backslash 1 \backslash G I N C-A C 27 \backslash F O p t \backslash U B 3 L Y P \backslash 6-31+G(d, p) \backslash C 9 H 11(2) \backslash B U C 562 \backslash 08-J u n-2006 \backslash 0 \backslash \backslash \#$

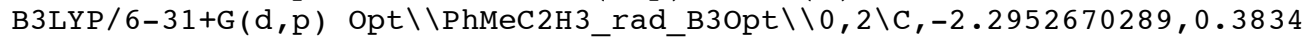
$694332,-0.0000407616 \backslash \mathrm{C},-1.911119 \overline{2} 151,-0.9644998985,0.0000778004 \backslash \mathrm{C},-0.5$ $673463615,-1.3191620436,0.0001541182 \backslash C, 0.4642442253,-0.3333294183,0.00$ $009552 \backslash \mathrm{C}, 0.0427676345,1.029906322,0.0000032276 \backslash \mathrm{C},-1.3036881134,1.37412$ $03149,-0.0000675135 \backslash \mathrm{H},-3.3462398701,0.6563675105,-0.0000988484 \backslash \mathrm{H},-2.66$ $9118183,-1.7432820159,0.0001167859 \backslash \mathrm{H},-0.3029197946,-2.3709942783,0.000$ $258709 \backslash \mathrm{H}, 0.7857087001,1.8200562571,0.0000119491 \backslash \mathrm{H},-1.5867521543,2.4233$ $661645,-0.0001355005 \backslash \mathrm{C}, 1.8490412265,-0.6930468672,0.000178456 \backslash \mathrm{C}, 2.2658$ $168643,-2.1393789341,0.0006097004 \backslash \mathrm{C}, 2.9169856097,0.3679150015,0.000196$ $2134 \backslash \mathrm{H}, 3.915511483,-0.0761814025,-0.000732568 \backslash \mathrm{H}, 2.8483598718,1.0245632$ $27,-0.8794340608 \backslash \mathrm{H}, 2.8494929587,1.0233501078,0.8808487726 \backslash \mathrm{H}, 3.35425320$ $42,-2.2375408525,0.0003382955 \backslash \mathrm{H}, 1.8874533785,-2.6793132478,0.880974432$ $5 \backslash \mathrm{H}, 1.8869445643,-2.6799943798,-0.8791057278 \backslash \backslash$ Version=IA64L-G0 3RevD 01 $\backslash$ State $=2-\mathrm{A} \backslash \mathrm{HF}=-349.5782245 \backslash \mathrm{S} 2=0.774607 \backslash \mathrm{S} 2-1=0 . \backslash \mathrm{S} 2 \mathrm{~A}=0.750418 \backslash \mathrm{RMSD}=5.742$ e-09 $\backslash$ RMSF $=2.978 e-05 \backslash$ Thermal $=0 . \backslash$ Dipole $=0.3112684,-0.0809246,0.0001518 \backslash P$ $\mathrm{G}=\mathrm{C} 01 \quad[\mathrm{X}(\mathrm{C} 9 \mathrm{H} 11)] \backslash \backslash @$

\section{2-Phenylpropanyl anion (AMS $\left.+\mathrm{H}^{-}\right)$}

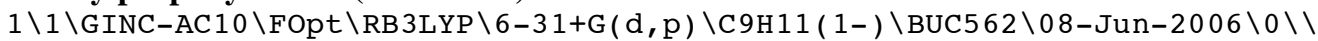
\# B3LYP $/ 6-31+\mathrm{G}(\mathrm{d}, \mathrm{p})$ Opt $\backslash \backslash \mathrm{PhCH}=\mathrm{CH} 3$ anion $\backslash \backslash-1,1 \backslash \mathrm{C},-2.6969051097,0.000015$ $6532,-0.0013406075 \backslash C,-1.9586302342,-1.1984459281,0.0923805283 \backslash C,-0.571$ $591839,-1.2147525376,0.087405202 \backslash C, 0.2246124311,-0.0021300509,-0.02858$ $3061 \backslash \mathrm{C},-0.5715002152,1.2143650237,-0.0935077773 \backslash \mathrm{C},-1.9585407196,1.1990$ $842825,-0.0861839887 \backslash \mathrm{H},-3.7839463491,0.0008211774,0.0089793647 \backslash \mathrm{H},-2.49$ $03150487,-2.148065172,0.1776574379 \backslash \mathrm{H},-0.0628073926,-2.1715229103,0.172$ $6250518 \backslash \mathrm{H},-0.0626379642,2.1731563094,-0.1509995215 \backslash \mathrm{H},-2.4901499877,2.1$ $509040282,-0.1424568777 \backslash \mathrm{C}, 1.618601886,-0.0047399583,-0.0631576989 \backslash \mathrm{C}, 2$. $3880450003,-1.2919002509,0.0897189034 \backslash \mathrm{C}, 2.3880905769,1.2908403631,-0.1$ $027025474 \backslash \mathrm{H}, 3.4636643149,1.1055407176,-0.2340492727 \backslash \mathrm{H}, 2.0824757035,1.9$ $555221629,-0.9325210657 \backslash \mathrm{H}, 2.2903722692,1.9130788462,0.8146549238 \backslash \mathrm{H}, 3.4$ $636376814,-1.128142805,-0.0675404553 \backslash \mathrm{H}, 2.2902203033,-1.7713301862,1.08$ $9148057 \backslash \mathrm{H}, 2.0824736931,-2.0721717651,-0.6324875953 \backslash \backslash$ Version=IA64L-G03R evD. $01 \backslash$ State $=1-\mathrm{A} \backslash \mathrm{HF}=-349.5998042 \backslash \mathrm{RMSD}=9.157 \mathrm{e}-09 \backslash \mathrm{RMSF}=2.487 \mathrm{e}-06 \backslash \mathrm{Thermal}$ $=0 . \backslash \mathrm{Dipole}=0.290126,0.0004522,0.0062167 \backslash \mathrm{PG}=\mathrm{C} 01[\mathrm{X}(\mathrm{C} 9 \mathrm{H} 11)] \backslash \backslash @$ 


\section{Isopropylbenzene (AMS $\left.+\mathrm{H}_{2}\right)$}

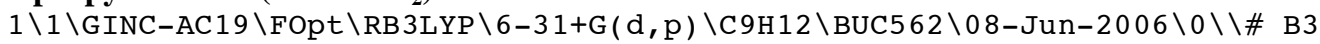

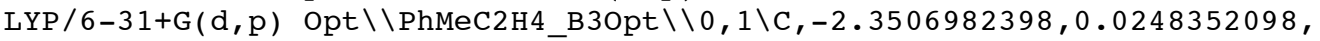
$0.2071905602 \backslash C_{,}-1.7008366727, \overline{1} .1729409298,-0.2500858176 \backslash C_{,}-0.319191577$ $9,1.1579602141,-0.465322217 \backslash \mathrm{C}, 0.4413419981,0.0036474789,-0.2305717606 \backslash$ $\mathrm{C},-0.2273047154,-1.1430665821,0.2285937082 \backslash \mathrm{C},-1.6066269086,-1.13482228$ $18,0.4458768242 \backslash \mathrm{H},-3.4235956208,0.031992535,0.3765450813 \backslash \mathrm{H},-2.26633744$ $31,2.0812875884,-0.4393621293 \backslash \mathrm{H}, 0.177029768,2.0581140314,-0.8210725795$ $\backslash \mathrm{H}, 0.3323415117,-2.0551263492,0.4191922436 \backslash \mathrm{H},-2.101101233,-2.034499523$ $3,0.8020697082 \backslash \mathrm{C}, 1.944460133,0.0020855017,-0.481019023 \backslash \mathrm{C}, 2.3276309108$, $-0.9316908216,-1.6463788069 \backslash \mathrm{H}, 2.2191505238,1.0238807797,-0.7749807359 \backslash$ $\mathrm{C}, 2.7474457022,-0.3422813179,0.7882706354 \backslash \mathrm{H}, 2.5465641683,-1.3664194151$ $, 1.1228026823 \backslash \mathrm{H}, 3.8233360203,-0.262234707,0.5953800278 \backslash \mathrm{H}, 2.4968007855$, $0.3335458416,1.6123792851 \backslash \mathrm{H}, 3.4027521163,-0.8722356193,-1.8512574399 \backslash \mathrm{H}$ , $2.0897053373,-1.9754074279,-1.411182195 \backslash \mathrm{H}, 1.7896924359,-0.6650510652$, $-2.5619110518 \backslash \backslash$ Version $=I A 64 \mathrm{~L}-\mathrm{G} 03$ RevD. $01 \backslash$ State $=1-\mathrm{A} \backslash \mathrm{HF}=-350.2229065 \backslash \mathrm{RMSD}$ $=4.327 \mathrm{e}-09 \backslash \mathrm{RMSF}=2.680 \mathrm{e}-05 \backslash \mathrm{Thermal}=0 . \backslash \mathrm{Dipole}=0.1510455,0.0043792,-0.028$ $5893 \backslash \mathrm{PG}=\mathrm{C} 01 \quad[\mathrm{X}(\mathrm{C} 9 \mathrm{H} 12)] \backslash \backslash$

\section{2,3-Dichloro-5-6-dicyano-1,4-benzoquinone (DDQ)}

$1 \backslash 1 \backslash G I N C-A C 51 \backslash F O p t \backslash R B 3 L Y P \backslash 6-31+G(d, p) \backslash C 8 C 12 N 202 \backslash B U C 562 \backslash 01-J u n-2006 \backslash 0 \backslash \backslash$

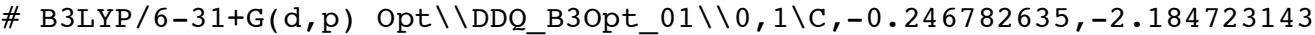
$, 0.0002484883 \backslash \mathrm{C}, 1.2484286141,-2.14 \overline{7} 4525325,0.0006874444 \backslash \mathrm{C}, 1.926952606$, $-0.9723056332,0.0000080497 \backslash \mathrm{C}, 1.2117142394,0.3412675834,-0.0011188142 \backslash \mathrm{C}$ $,-0.292653712,0.3110188398,-0.0015436206 \backslash C,-0.9726814939,-0.8667302319$ $,-0.0009433244 \backslash \mathrm{Cl}, 2.0259710479,-3.6787339512,0.0020893264 \backslash \mathrm{Cl}, 3.6418601$ $636,-0.8801560582,0.0003939568 \backslash \mathrm{C},-0.9512754363,1.5777275149,-0.0026260$ $188 \backslash \mathrm{C},-2.3989990846,-0.9296053549,-0.0013674157 \backslash 0,-0.8702147904,-3.227$ $2826871,0.0008245285 \backslash 0,1.8029558028,1.4024208599,-0.0017235913 \backslash \mathrm{N},-1.49$ $12511039,2.6075133414,-0.0035218324 \backslash N,-3.5608112176,-0.9767835473,-0.0$ $017071768 \backslash \backslash$ Version=IA64L-G03RevD.01 \State $=1-\mathrm{A} \backslash \mathrm{HF}=-1485.1153261 \backslash \mathrm{RMSD}=8$. $419 \mathrm{e}-09 \backslash \mathrm{RMSF}=5.475 \mathrm{e}-05 \backslash \mathrm{Thermal}=0 . \backslash \mathrm{Dipole}=1.4895781,-0.8600931,0.001080$ $3 \backslash \mathrm{PG}=\mathrm{C} 01 \quad[\mathrm{X}(\mathrm{C} 8 \mathrm{C} 12 \mathrm{~N} 2 \mathrm{O} 2)] \backslash \backslash @$

\section{2,3-Dichloro-5-6-dicyano- $O$-hydro-1,4-benzoquinonyl radial (DDQ + H•)}

$1 \backslash 1 \backslash G I N C-A C 16 \backslash F O p t \backslash U B 3 L Y P \backslash 6-31+G(d, p) \backslash C 8 H 1 C 12 N 2 O 2(2) \backslash B U C 562 \backslash 01-J u n-200$

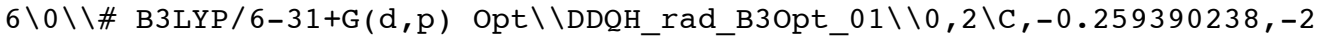
$.1988125286,0.0002361169 \backslash \mathrm{C}, 1.21478 \overline{8} 250 \overline{2},-2.1 \overline{5} 34317064,0.0006603812 \backslash \mathrm{C}, 1$ $.8877328752,-0.9490217584,0.000016434 \backslash C, 1.1796600839,0.2847433168,-0.0$ $010796854 \backslash \mathrm{C},-0.2406106074,0.299650737,-0.0015327265 \backslash \mathrm{C},-0.949244211,-0$. $9029952985,-0.0008964032 \backslash \mathrm{Cl}, 2.0395051546,-3.6554219747,0.0019785226 \backslash \mathrm{Cl}$ , $3.6302667507,-0.8567794003,0.0004898701 \backslash \mathrm{C},-0.9084475661,1.5648373921$, $-0.0026431583 \backslash \mathrm{C},-2.3727805289,-0.9207005538,-0.001318254 \backslash 0,-0.88077986$ $15,-3.269749743,0.0008014257 \backslash 0,1.8010609598,1.4646082109,-0.0017173244$ $\backslash \mathrm{H}, 2.7669691505,1.3428851755,-0.0013448909 \backslash \mathrm{N},-1.4458347683,2.595713219$ $2,-0.0035517244 \backslash \mathrm{N},-3.536746444,-0.9283770878,-0.0016645836 \backslash \backslash$ Version $=I A$ $64 \mathrm{~L}-\mathrm{G} 03$ RevD. $01 \backslash$ State $=2-\mathrm{A} \backslash \mathrm{HF}=-1485.7310584 \backslash \mathrm{S} 2=0.779862 \backslash \mathrm{S} 2-1=0 . \backslash \mathrm{S} 2 \mathrm{~A}=0.75$ $0653 \backslash \mathrm{RMSD}=5.055 \mathrm{e}-09 \backslash \mathrm{RMSF}=2.687 \mathrm{e}-05 \backslash \mathrm{Thermal}=0 . \backslash \mathrm{Dipole}=3.0325184,0.01823$ $12,0.0009212 \backslash \mathrm{PG}=\mathrm{C} 01 \quad[\mathrm{X}(\mathrm{C} 8 \mathrm{H} 1 \mathrm{Cl} 2 \mathrm{~N} 2 \mathrm{O} 2)] \backslash \backslash @$ 


\section{2,3-Dichloro-5-6-dicyano- $O$-hydro-1,4-benzoquinonyl anion (DDQ $\left.+\mathrm{H}^{-}\right)$}

$1 \backslash 1 \backslash \mathrm{GINC}-\mathrm{AC} 49 \backslash \mathrm{FOpt} \backslash \mathrm{RB} 3 \mathrm{LYP} \backslash 6-31+\mathrm{G}(\mathrm{d}, \mathrm{p}) \backslash \mathrm{C} 8 \mathrm{H} 1 \mathrm{Cl} 2 \mathrm{~N} 2 \mathrm{O} 2(1-) \backslash \mathrm{BUC5} 62 \backslash 01-\mathrm{Jun}-20$

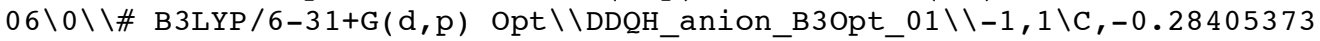
$49,-2.2295629206,0.0002545695 \backslash C, 1.1800299923,-2.1379411712,0.000640309$ $2 \backslash \mathrm{C}, 1.8541350087,-0.9281070144,-0.0000292475 \backslash \mathrm{C}, 1.1770928834,0.30934803$ $17,-0.0011075803 \backslash \mathrm{C},-0.2192042514,0.2951549038,-0.0015301228 \backslash \mathrm{C},-0.94126$ $7447,-0.9321626937,-0.0008754274 \backslash \mathrm{Cl}, 2.0502546417,-3.6490101022,0.00198$ $13802 \backslash \mathrm{Cl}, 3.6259265839,-0.8547899395,0.0004607654 \backslash \mathrm{C},-0.9040884477,1.551$ $9195806,-0.002622684 \backslash \mathrm{C},-2.3636586454,-0.9208340227,-0.0013313697 \backslash 0,-0$. $8894824168,-3.3224244696,0.0008596141 \backslash 0,1.8344343244,1.5148284569,-0.0$ $017548661 \backslash \mathrm{H}, 2.78714429,1.3402311159,-0.001280065 \backslash \mathrm{N},-1.449910114,2.5813$ $369745,-0.0035227089 \backslash N,-3.5312036673,-0.9008387296,-0.0017085666 \backslash \backslash$ Vers ion $=I A 64 \mathrm{~L}-\mathrm{G} 03 \mathrm{RevD} .01 \backslash$ State $=1-\mathrm{A} \backslash \mathrm{HF}=-1485.8664327 \backslash \mathrm{RMSD}=9.255 \mathrm{e}-09 \backslash \mathrm{RMSF}=4$. $694 \mathrm{e}-05 \backslash \mathrm{Thermal}=0 . \backslash \mathrm{Dipole}=3.9350807,0.230251,0.0010701 \backslash \mathrm{PG}=\mathrm{C} 01 \quad[\mathrm{X}(\mathrm{C} 8 \mathrm{H} 1 \mathrm{C}$ $12 \mathrm{~N} 2 \mathrm{O} 2)] \backslash \backslash @$

\section{2,3-Dichloro-5-6-dicyano- $O, O^{\prime}$-dihydro-1,4-benzoquinone (DDQ $+\mathrm{H}_{2}$ )}

$1 \backslash 1 \backslash$ GINC-AC3 $\backslash$ FOpt $\backslash$ RB $3 L Y P \backslash 6-31+G(d, p) \backslash C 8 H 2 C 12 N 2 O 2 \backslash B U C 562 \backslash 01-J u n-2006 \backslash 0 \backslash$

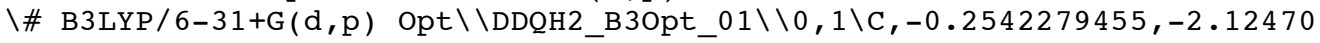
$07722,0.0001918601 \backslash \mathrm{C}, 1.15479513 \overline{9} 4,-2 . \overline{1} 368644206,0.0006365008 \backslash \mathrm{C}, 1.85993$ $22421,-0.9302265264,-0.0000035501 \backslash C, 1.1938568626,0.312324685,-0.001103$ $2075 \backslash C,-0.2076398445,0.3314922078,-0.0015449197 \backslash C,-0.9261307703,-0.890$ $0540249,-0.0008911736 \backslash \mathrm{Cl}, 1.9730333494,-3.6599270415,0.0019910478 \backslash \mathrm{Cl}, 3$. $6024308013,-0.9064977463,0.0005294639 \backslash \mathrm{C},-0.8967911357,1.5825261923,-0$. $0026491523 \backslash \mathrm{C},-2.3534008027,-0.9269590639,-0.00130119 \backslash 0,-0.8950039461,-$ $3.3118258987,0.0008354994 \backslash 0,1.8429314387,1.4939953812,-0.0017573799 \backslash \mathrm{H}$, $2.802686779,1.350050079,-0.0013443757 \backslash \mathrm{H},-1.8579736952,-3.1862632181,0$. $00044771 \backslash \mathrm{N},-1.4642967093,2.5978330045,-0.003552858 \backslash \mathrm{N},-3.5081907634,-1$. $0784838374,-0.0015462752 \backslash \backslash$ Version=IA64L-G03RevD.01 \State $=1-A \backslash H F=-1486$. $3749866 \backslash \mathrm{RMSD}=5.724 \mathrm{e}-09 \backslash \mathrm{RMSF}=4.934 \mathrm{e}-05 \backslash \mathrm{Thermal}=0 . \backslash \mathrm{Dipole}=1.8510123,-1.0$ $760805,0.001344 \backslash \mathrm{PG}=\mathrm{C} 01 \quad[\mathrm{X}(\mathrm{C} 8 \mathrm{H} 2 \mathrm{C} 12 \mathrm{~N} 2 \mathrm{O} 2)] \backslash \backslash @$

\section{para-Benzoquinone (PBQ)}

$1 \backslash 1 \backslash \mathrm{GINC}-\mathrm{AC} 6 \backslash$ FOpt $\backslash \mathrm{RB} 3 \mathrm{LYP} \backslash 6-31+\mathrm{G}(\mathrm{d}, \mathrm{p}) \backslash \mathrm{C} 6 \mathrm{H} 4 \mathrm{O} 2 \backslash \mathrm{BUC5} 62 \backslash 22-\mathrm{Sep}-2005 \backslash 0 \backslash \backslash \#$ B3

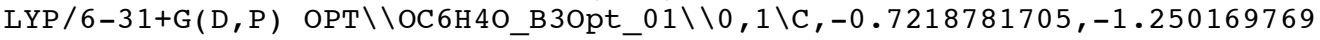
$2,0.0005390372 \backslash \mathrm{C}, 0.76429555 \overline{6} 1,-1 . \overline{2} 176957021,0.0010130365 \backslash \mathrm{C}, 1.436697596$ $1,-0.0532086675,0.000485951 \backslash \mathrm{C}, 0.7218723156,1.250175787,-0.0005113295 \backslash \mathrm{C}$ $,-0.7642904877,1.2176935118,-0.0010323495 \backslash \mathrm{C},-1.4366903539,0.0532045337$ $,-0.0004931296 \backslash \mathrm{H}, 1.2628508008,-2.1827465402,0.0017812373 \backslash \mathrm{H}, 2.521739205$ $2,-0.0025583987,0.0007839582 \backslash \mathrm{H},-1.2628615359,2.1827363874,-0.001837106$ $5 \backslash \mathrm{H},-2.5217324628,0.0025713487,-0.0007901006 \backslash \mathrm{O},-1.335465628,-2.3127989$ $341,0.0010199374 \backslash 0,1.3354612853,2.3127988142,-0.0010130981 \backslash \backslash$ Version $=I A$ $64 \mathrm{~L}-\mathrm{G} 03$ RevC $.02 \backslash$ State $=1-\mathrm{A} \backslash \mathrm{HF}=-381.4765024 \backslash \mathrm{RMSD}=6.628 \mathrm{e}-09 \backslash \mathrm{RMSF}=6.198 \mathrm{e}-05$ $\backslash$ Dipole $=-0.0000067,0.0000189,0 . \backslash \mathrm{PG}=\mathrm{C} 01[\mathrm{X}(\mathrm{C} 6 \mathrm{H} 4 \mathrm{O} 2)] \backslash \backslash @$

\section{$O$-hydro-para-Benzoquinonyl radical (PBQ + H•)}

$1 \backslash 1 \backslash G I N C-A C 17 \backslash F O p t \backslash U B 3 L Y P \backslash 6-31+G(d, p) \backslash C 6 H 5 O 2(2) \backslash B U C 562 \backslash 22-$ Sep-2005 $2 \backslash \backslash$

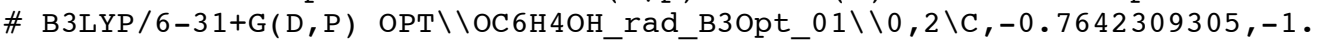
$2953487031,0.0005746392 \backslash \mathrm{C}, 0.68895181 \overline{3},-1.2618187951,0.0010226881 \backslash \mathrm{C}, 1.3$ $770858644,-0.0694212818,0.0004817642 \backslash \mathrm{C}, 0.6679543728,1.153635258,-0.000$ $5238044 \backslash \mathrm{C},-0.7467053334,1.1724498357,-0.0009784559 \backslash \mathrm{C},-1.445592665,-0.0$ $10475868,-0.0004489058 \backslash \mathrm{H}, 1.2081200722,-2.2148253785,0.0017944628 \backslash \mathrm{H}, 2.4$ $65526195,-0.0579692698,0.0008250097 \backslash H,-1.249870896,2.1342989674,-0.001$ $7448377 \backslash \mathrm{H},-2.5307690538,-0.0256852227,-0.0007804242 \backslash \mathrm{O},-1.3980376232,-2$ $.3825231734,0.0010622085 \backslash 0,1.2962234219,2.3562603113,-0.0010790489 \backslash \mathrm{H}, 2$ $.2567285657,2.2401611261,-0.0007270394 \backslash$ \Version=IA64L-G03RevC.02\State $=2-\mathrm{A} \backslash \mathrm{HF}=-382.081279 \backslash \mathrm{S} 2=0.777548 \backslash \mathrm{S} 2-1=0 . \backslash \mathrm{S} 2 \mathrm{~A}=0.750516 \backslash \mathrm{RMSD}=5.178 \mathrm{e}-09 \backslash \mathrm{RM}$ $\mathrm{SF}=3.539 \mathrm{e}-05 \backslash \mathrm{Dipole}=1.4170368,1.3083079,-0.000392 \backslash \mathrm{PG}=\mathrm{C} 01 \quad \mathrm{X}(\mathrm{C} 6 \mathrm{H} 5 \mathrm{O} 2)] \backslash \backslash$ a 


\section{$O$-hydro-para-Benzoquinonyl anion (PBQ $\left.+\mathrm{H}^{-}\right)$}

$1 \backslash 1 \backslash G I N C-A C 17 \backslash F O p t \backslash R B 3 L Y P \backslash 6-31+G(d, p) \backslash C 6 H 5 O 2(1-) \backslash B U C 562 \backslash 22-$ Sep-2005 $0 \backslash$

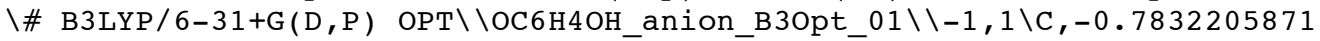
$,-1.3312715549,0.0005461599 \backslash \mathrm{C}, 0.6 \overline{5} 95091068,-1.2562259709,0.0009669223 \backslash$ $\mathrm{C}, 1.3542734353,-0.0430884475,0.0005131818 \backslash \mathrm{C}, 0.6724809687,1.1766376432$, $-0.0004735651 \backslash \mathrm{C},-0.7242304276,1.1683450092,-0.0009464504 \backslash \mathrm{C},-1.42835882$ $36,-0.0370492114,-0.0004608542 \backslash \mathrm{H}, 1.208362099,-2.1962113226,0.001779646$ $5 \backslash \mathrm{H}, 2.4481312015,-0.0483872293,0.00092387 \backslash \mathrm{H},-1.255865385,2.1191423041$, $-0.0017226775 \backslash \mathrm{H},-2.5169489963,-0.026924393,-0.0008436322 \backslash 0,-1.42776141$ $52,-2.4367235435,0.0009570953 \backslash 0,1.343249357,2.4186969885,-0.0009406307$ $\backslash \mathrm{H}, 2.2896955121,2.2325082753,-0.0011412895 \backslash \backslash$ Version=IA64L-G03RevC.02 $\backslash \mathrm{S}$ tate $=1-\mathrm{A} \backslash \mathrm{HF}=-382.1488054 \backslash \mathrm{RMSD}=5.647 \mathrm{e}-09 \backslash \mathrm{RMSF}=7.725 \mathrm{e}-05 \backslash \mathrm{Dipole}=1.742999$ $5,1.9545204,-0.0009213 \backslash \mathrm{PG}=\mathrm{C} 01 \quad[\mathrm{X}(\mathrm{C} 6 \mathrm{H} 5 \mathrm{O} 2)] \backslash \backslash \mathrm{a}$

\section{$O, O^{\prime}$-dihydro-para-Benzoquinone (PBQ $\left.+\mathrm{H}_{2}\right)$}

$1 \backslash 1 \backslash G I N C-A C 19 \backslash F O p t \backslash R B 3 L Y P \backslash 6-31+G(d, p) \backslash C 6 H 602 \backslash B U C 562 \backslash 22-S e p-2005 \backslash 0 \backslash \backslash \# B$ $3 \mathrm{LYP} / 6-31+\mathrm{G}(\mathrm{D}, \mathrm{P}) \quad \mathrm{OPT} \backslash \backslash \mathrm{HOC} 6 \mathrm{H} 4 \mathrm{OH} \_\mathrm{B} 3 \mathrm{Opt} \_01 \backslash \backslash 0,1 \backslash \mathrm{C},-0.711567141,-1.2062177$ $377,0.0005450061 \backslash \mathrm{C}, 0.684796938 \overline{8},-1.2 \overline{1} 82442031,0.0009921675 \backslash \mathrm{C}, 1.3931731$ $175,-0.0150803226,0.0004546219 \backslash \mathrm{C}, 0.7115373457,1.2062381981,-0.00054269$ $91 \backslash C,-0.6847917569,1.218261145,-0.0009901581 \backslash C,-1.3931878404,0.0150686$ $097,-0.0004469925 \backslash \mathrm{H}, 1.2066939809,-2.1693747781,0.0017595744 \backslash \mathrm{H}, 2.480809$ $2332,-0.033444077,0.0008241397 \backslash \mathrm{H},-1.206745734,2.1693610172,-0.00176735$ $79 \backslash \mathrm{H},-2.4808211462,0.0334860285,-0.0008037751 \backslash 0,-1.356061917,-2.422288$ $9707,0.0011037784 \backslash \mathrm{O}, 1.3560962671,2.4222749282,-0.0010782427 \backslash \mathrm{H}, 2.312449$ $8912,2.2877763476,-0.0010256362 \backslash \mathrm{H},-2.3124250075,-2.2878463351,0.000737$ $0948 \backslash \backslash$ Version=IA64L-G03RevC.02 \State $=1-A \backslash H F=-382.716646 \backslash$ RMSD $=5.730 e-09$ $\backslash \mathrm{RMSF}=1.208 \mathrm{e}-04 \backslash \mathrm{Dipole}=-0.0000179,-0.0000511,-0.0002017 \backslash \mathrm{PG}=\mathrm{C} 01 \quad[\mathrm{X}(\mathrm{C} 6 \mathrm{H} 6$ $02)] \backslash \backslash @$

\section{The ACD/AMS reaction}

\section{ACD/AMS TS1 (RB3-LYP, gas-phase)}

$1 \backslash 1 \backslash G I N C-A C 27 \backslash F T S \backslash R B 3 L Y P \backslash 6-31+G(d, p) \backslash C 22 H 21 N 1 \backslash B U C 562 \backslash 15-J u n-2006 \backslash 0 \backslash \backslash \#$ B3LYP $/ 6-31+G(d, p)$ Opt $=($ TS ,CalcFC,NoEigenTest $) \backslash \backslash$ PhMeC2H3_TS_C13H9NH_B3O

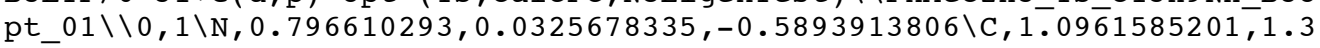
$68 \overline{3} 755322,-0.4005371454 \backslash \mathrm{C}, 2.4136222879,1.7388358388,0.028010171 \backslash \mathrm{C}, 3.37$ $6135558,0.729445029,0.2181830432 \backslash \mathrm{C}, 3.0755795382,-0.6191682325,-0.05231$ $65111 \backslash \mathrm{C}, 1.7496965969,-0.9617226685,-0.4759447237 \backslash \mathrm{H}, 4.37378023,0.997895$ $9289,0.554029098 \backslash \mathrm{H},-0.206655298,-0.2485579509,-0.4912152203 \backslash \mathrm{C},-1.78479$ $25748,-0.7282295848,0.7035857877 \backslash \mathrm{C},-2.5781315196,-0.4826153198,-0.4595$ $574529 \backslash \mathrm{C},-3.2167145507,0.7842994531,-0.6747607454 \backslash \mathrm{C},-3.9533885877,1.05$ $13259699,-1.8229253325 \backslash \mathrm{C},-4.1036871061,0.0856760861,-2.826866994 \backslash \mathrm{C},-3$. $5035249251,-1.1690207592,-2.642417226 \backslash C,-2.7685429536,-1.4520240791,-1$ $.4993348334 \backslash \mathrm{C},-1.3997803938,-2.1153313857,1.143199835 \backslash \mathrm{C},-1.675574041,0$ $.3161324127,1.785061041 \backslash \mathrm{H},-3.1509224167,1.5495204379,0.0909987765 \backslash \mathrm{H},-4$ $.427595674,2.0236421912,-1.9333586946 \backslash \mathrm{H},-4.6789328736,0.299501132,-3.7$ $220825041 \backslash \mathrm{H},-3.615523534,-1.937893099,-3.4028524648 \backslash \mathrm{H},-2.3314594824,-2$ $.4398556255,-1.4004985684 \backslash \mathrm{H},-0.3610647928,-2.1546515792,1.5024375749 \backslash \mathrm{H}$ $,-2.0255131101,-2.4383396253,1.9930214005 \backslash \mathrm{H},-1.5077490443,-2.876659508$ $, 0.3684887041 \backslash \mathrm{H},-2.637098051,0.4839271837,2.3009265908 \backslash \mathrm{H},-0.9548881164$ $, 0.0051116363,2.5485505974 \backslash \mathrm{H},-1.3493865256,1.2962019938,1.4135273065 \backslash \mathrm{C}$ $, 4.0319993803,-1.6698614079,0.0567724189 \backslash \mathrm{C}, 3.7016483553,-2.9712020592$, $-0.2617478302 \backslash \mathrm{C}, 2.3965671855,-3.2859506662,-0.7033597048 \backslash \mathrm{C}, 1.433196228$ $2,-2.2942732264,-0.8053727026 \backslash C, 0.126711392,2.3590637343,-0.6564092231$ $\backslash C, 0.4350608028,3.6975526568,-0.4646333538 \backslash C, 1.7176435295,4.0796241849$ $,-0.010686208 \backslash \mathrm{C}, 2.6839207419,3.1236508505,0.2273207053 \backslash \mathrm{H}, 5.034619952,-$ $1.4215246731,0.3936245305 \backslash \mathrm{H}, 4.4464622181,-3.7567861384,-0.1779078093 \backslash \mathrm{H}$ $, 2.1447099209,-4.3087421044,-0.9652674392 \backslash \mathrm{H}, 0.4334563211,-2.5281504486$ $,-1.1549769738 \backslash \mathrm{H}, 3.6739145287,3.4120110228,0.5695808131 \backslash \mathrm{H}, 1.944796946$, $5.1305263001,0.1412502207 \backslash \mathrm{H},-0.3139192554,4.4550861707,-0.6730558461 \backslash \mathrm{H}$ $,-0.8514546995,2.0604425624,-1.0203557267 \backslash \backslash$ Version=IA64L-G03RevD.01\S ate $=1-\mathrm{A} \backslash \mathrm{HF}=-905.7528703 \backslash \mathrm{RMSD}=3.381 \mathrm{e}-09 \backslash \mathrm{RMSF}=4.938 \mathrm{e}-06 \backslash \mathrm{Thermal}=0 . \backslash \mathrm{Dipol}$ $\mathrm{e}=1.0772828,0.0824831,0.267643 \backslash \mathrm{PG}=\mathrm{C} 01 \quad[\mathrm{X}(\mathrm{C} 22 \mathrm{H} 21 \mathrm{~N} 1)] \backslash \backslash @$ 


\section{ACD/AMS TS1 (UB3-LYP, gas phase)}

$1 \backslash 1 \backslash G I N C-A C 10 \backslash F T S \backslash U B 3 L Y P \backslash 6-31+G(d, p) \backslash C 22 H 21 N 1 \backslash B U C 562 \backslash 16-J u n-2006 \backslash 0 \backslash \backslash \#$ $\mathrm{UB} 3 \mathrm{LYP} / 6-31+\mathrm{G}(\mathrm{d}, \mathrm{p})$ Guess $=($ Mix, Always $)$ Opt $=(\mathrm{TS}, \mathrm{CalcFC}$, NoEigenTest $) \backslash \backslash \mathrm{PhM}$

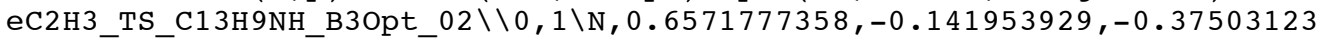
$2 \backslash \mathrm{C}, 0.90 \overline{5} 9672476,1.217 \overline{9} 312755,-0.3521831043 \backslash \mathrm{C}, 2.2238328579,1.720883303$ $5,-0.0898980524 \backslash \mathrm{C}, 3.2713068353,0.8029141247,0.1141763987 \backslash \mathrm{C}, 3.037927827$ $6,-0.581421706,0.0087071581 \backslash \mathrm{C}, 1.7040140621,-1.037295628,-0.2582158642 \backslash$ $\mathrm{H}, 4.2733447845,1.1663260834,0.325522765 \backslash \mathrm{H},-0.3206264606,-0.4537341432$, $0.1495783114 \backslash \mathrm{C},-1.4995548748,-0.7571349542,1.2445540745 \backslash \mathrm{C},-2.651577058$ $6,-0.473786202,0.388655447 \backslash \mathrm{C},-3.3521277588,0.7609640406,0.4572902289 \backslash \mathrm{C}$ $,-4.4211796303,1.0477553455,-0.3898981237 \backslash \mathrm{C},-4.8409374199,0.1214520431$ $,-1.3497802544 \backslash \mathrm{C},-4.1700082133,-1.1042153297,-1.4404961222 \backslash \mathrm{C},-3.104973$ $8563,-1.3965497779,-0.5938318624 \backslash C,-1.2385008234,-2.1963266609,1.65098$ $64954 \backslash \mathrm{C},-1.1644799685,0.227246649,2.3484667733 \backslash \mathrm{H},-3.0661808695,1.49673$ $22214,1.2004816641 \backslash \mathrm{H},-4.9363811018,2.00013811,-0.2931068151 \backslash \mathrm{H},-5.67406$ $25239,0.3458472267,-2.0089684005 \backslash \mathrm{H},-4.4810350984,-1.8395106878,-2.1780$ $466156 \backslash \mathrm{H},-2.6105680951,-2.3564519437,-0.6986181699 \backslash \mathrm{H},-0.2180308426,-2$. $3213359731,2.0297015277 \backslash \mathrm{H},-1.919906994,-2.4965016477,2.4653867909 \backslash \mathrm{H},-1$ $.3802586381,-2.9183952348,0.8431980205 \backslash \mathrm{H},-1.9453460099,0.2528262177,3$. $1274850407 \backslash \mathrm{H},-0.2297373318,-0.0592201158,2.8425736568 \backslash \mathrm{H},-1.0379596709$, $1.2531634715,1.9869985814 \backslash \mathrm{C}, 4.0736993328,-1.5524060122,0.1331912786 \backslash \mathrm{C}$, $3.8105440176,-2.8968388493,-0.0194649178 \backslash \mathrm{C}, 2.4958384957,-3.3328963354$, $-0.3101699799 \backslash \mathrm{C}, 1.4638751621,-2.4188873546,-0.4295224775 \backslash \mathrm{C},-0.14833692$ $99,2.1234009246,-0.609779891 \backslash C, 0.080297926,3.4881519532,-0.5852059778 \backslash$ $\mathrm{C}, 1.370786185,3.9973452331,-0.3050990026 \backslash \mathrm{C}, 2.4172107587,3.1326741179$,$0.0656535016 \backslash \mathrm{H}, 5.081266028,-1.2078558164,0.3503099722 \backslash \mathrm{H}, 4.6116890458$,$3.6234711899,0.0762677597 \backslash \mathrm{H}, 2.295884269,-4.3912331401,-0.4470856594 \backslash \mathrm{H}$, $0.4584915469,-2.7487415495,-0.6661788089 \backslash \mathrm{H}, 3.4126578635,3.5144621427,0$ $.1450775826 \backslash \mathrm{H}, 1.5356572022,5.0704851685,-0.2867251033 \backslash \mathrm{H},-0.7373636768$, $4.1724560813,-0.7903356037 \backslash \mathrm{H},-1.1344563368,1.7312774475,-0.8342709872 \backslash$ $\backslash$ Version=IA64L-G03RevD. 01 \State $=1-\mathrm{A} \backslash \mathrm{HF}=-905.7568493 \backslash \mathrm{S} 2=0.411422 \backslash \mathrm{S} 2-1=0$ $. \backslash \mathrm{S} 2 \mathrm{~A}=0.031211 \backslash \mathrm{RMSD}=9.470 \mathrm{e}-09 \backslash \mathrm{RMSF}=4.974 \mathrm{e}-06 \backslash \mathrm{Thermal}=0 . \backslash \mathrm{Dipole}=0.84973$ $07,0.120607,0.0875675 \backslash \mathrm{PG}=\mathrm{C} 01 \quad[\mathrm{X}(\mathrm{C} 22 \mathrm{H} 21 \mathrm{~N} 1)] \backslash \backslash @$ 


\section{ACD/AMS TS1 (UB3-LYP, heptane)}

$1 \backslash 1 \backslash G I N C-A C 41 \backslash F T S \backslash U B 3 L Y P \backslash 6-31+G(d, p) \backslash C 22 H 21 N 1 \backslash B U C 562 \backslash 13-J u n-2006 \backslash 0 \backslash \backslash \#$ $\mathrm{UB} 3 \mathrm{LYP} / 6-31+\mathrm{G}(\mathrm{d}, \mathrm{p})$ Guess=(Mix,Always) Opt=(TS,CalcFC,NoEigenTest) SCRF $=($ Read, IEFPCM, Solvent $=$ Heptane $) \backslash \backslash$ PhMeC2H2_TS_C13H10NH_B3Opt_Heptane_02 $\backslash 0,1 \backslash \mathrm{H}, 0.2313077261,-0.9900259026,0.4264 \overline{2} 45 \overline{3} 15 \backslash \mathrm{C}, 1.1 \overline{4} 01865 \overline{1} 36,-0.5 \overline{6} 493$ $05971,-0.8175099877 \backslash \mathrm{C}, 0.7184189085,0.8169298098,-0.8514363654 \backslash \mathrm{C}, 1.5498$ $9171,1.8057422332,-0.253942352 \backslash \mathrm{N}, 2.7812372071,1.4174795766,0.260353843$ $3 \backslash \mathrm{C}, 3.3185491337,0.1426741425,0.1152026617 \backslash \mathrm{C}, 2.5102624147,-0.875551850$ $7,-0.45773192 \backslash \mathrm{H}, 0.6371927407,-1.2563191173,-1.4910581651 \backslash \mathrm{H}, 3.381793857$ $5,2.1395414761,0.6348530429 \backslash \mathrm{C},-0.5078560281,-1.2692653804,1.3743114024$ $\backslash \mathrm{H}, 0.178508586,-1.1276247705,2.2132804072 \backslash \mathrm{H},-0.6902207455,-2.331872022$ $9,1.1989163401 \backslash \mathrm{C},-1.6586202542,-0.4151216984,1.4012530743 \backslash \mathrm{C},-1.5927647$ $416,0.8609467096,2.2006313051 \backslash \mathrm{C},-2.8680937704,-0.7279353582,0.66195827$ $34 \backslash C,-4.0186261512,0.1011911688,0.7480771905 \backslash C,-2.9696254202,-1.867263$ $4311,-0.1826321034 \backslash \mathrm{C},-5.1863290462,-0.1945791879,0.0483371129 \backslash \mathrm{H},-3.997$ $2225668,0.9861010743,1.3739564452 \backslash C,-4.1369204784,-2.1558616699,-0.881$ $8156011 \backslash \mathrm{H},-2.1197298144,-2.5313658661,-0.2965554982 \backslash \mathrm{C},-5.2593210949,-1$ $.3241945659,-0.7735212606 \backslash \mathrm{H},-6.0462113656,0.4632277086,0.1445519333 \backslash \mathrm{H}$, $-4.1734014326,-3.0361488804,-1.5183471254 \backslash \mathrm{H},-6.1698251716,-1.552399265$ $8,-1.3195820617 \backslash \mathrm{H},-2.3472630602,0.8737062524,3.0007874614 \backslash \mathrm{H},-0.6132114$ $375,0.9737348684,2.671848502 \backslash \mathrm{H},-1.7696654353,1.7529008936,1.5833603133$ $\backslash \mathrm{C}, 4.6310850823,-0.1342977529,0.5209976221 \backslash \mathrm{C}, 5.1574658154,-1.417104257$ $3,0.3661378837 \backslash \mathrm{C}, 4.3788785984,-2.432580595,-0.2047209108 \backslash \mathrm{C}, 3.074488579$ $5,-2.1573126005,-0.6098762331 \backslash \mathrm{C}, 1.1280033989,3.1385270518,-0.183124861$ $2 \backslash \mathrm{C},-0.1242058548,3.5047841836,-0.6836476048 \backslash \mathrm{C},-0.95786556,2.547329993$ $5,-1.2726690832 \backslash \mathrm{C},-0.5372494753,1.2194542648,-1.3481998444 \backslash \mathrm{H}, 5.2373480$ $576,0.6582459592,0.9537942182 \backslash \mathrm{H}, 6.1752309014,-1.620521619,0.6853396373$ $\backslash \mathrm{H}, 4.7889421596,-3.4297655644,-0.332212312 \backslash \mathrm{H}, 2.466242029,-2.9416850965$ $,-1.0536191236 \backslash \mathrm{H}, 1.7778151844,3.8849730208,0.2678408373 \backslash \mathrm{H},-0.441910716$ $3,4.5413649603,-0.6190401933 \backslash \mathrm{H},-1.9294680058,2.8325101012,-1.663734641$ $6 \backslash \mathrm{H},-1.1827229774,0.4702276016,-1.7971597905 \backslash \backslash$ Version=IA64L-G0 3RevD. 01 $\backslash$ State $=1-\mathrm{A} \backslash \mathrm{HF}=-905.7590428 \backslash \mathrm{S} 2=0.178354 \backslash \mathrm{S} 2-1=0 . \backslash \mathrm{S} 2 \mathrm{~A}=0.005316 \backslash \mathrm{RMSD}=6.662$ $e-09 \backslash \mathrm{RMSF}=5.677 \mathrm{e}-06 \backslash \mathrm{Thermal}=0 . \backslash \mathrm{Dipole}=0.7031549,0.5160233,0.3061348 \backslash \mathrm{PG}$ $=\mathrm{C} 01[\mathrm{X}(\mathrm{C} 22 \mathrm{H} 21 \mathrm{~N} 1)] \backslash \backslash \mathrm{Q}$ 


\section{ACD/AMS TS1 (UB3-LYP, ether)}

$1 \backslash 1 \backslash G I N C-A C 29 \backslash F T S \backslash U B 3 L Y P \backslash 6-31+G(d, p) \backslash C 22 H 21 N 1 \backslash B U C 562 \backslash 23-J u n-2006 \backslash 0 \backslash \backslash \#$ $\mathrm{UB} 3 \mathrm{LYP} / 6-31+\mathrm{G}(\mathrm{d}, \mathrm{p})$ Guess=(Mix,Always) Opt=(TS, CalcFC, NoEigenTest, Loose ) $\mathrm{SCRF}=($ Read, IEFPCM, Solvent $=$ Ether $) \backslash \backslash \mathrm{PhMeC} 2 \mathrm{H} 2$ TS C13H10NH B3Opt Ether 0 $2 \backslash \backslash 0,1 \backslash \mathrm{H}, 0.2203636959,-1.0225430642,0.355949 \overline{6} 23 \overline{3} \backslash \mathrm{C}, 1.143 \overline{4} 13927 \overline{7},-0.5 \overline{5} 1$ $7455197,-0.858472445 \backslash \mathrm{C}, 0.7394983212,0.8355502859,-0.8302598883 \backslash \mathrm{C}, 1.577$ $6476502,1.7844480183,-0.1786072274 \backslash \mathrm{N}, 2.7994989612,1.359135812,0.326735$ $4935 \backslash \mathrm{C}, 3.3200373959,0.0853113502,0.1292218514 \backslash \mathrm{C}, 2.5057390128,-0.895437$ $1923,-0.4992151849 \backslash \mathrm{H}, 0.6387802335,-1.2056069075,-1.5682007172 \backslash \mathrm{H}, 3.4046$ $078501,2.0558482328,0.7479848495 \backslash \mathrm{C},-0.5236404604,-1.3383096505,1.28846$ $10684 \backslash \mathrm{H}, 0.1685649393,-1.2577914123,2.1310716771 \backslash \mathrm{H},-0.728964045,-2.3853$ $924803,1.0543441793 \backslash \mathrm{C},-1.6553147093,-0.4619188846,1.3675530892 \backslash \mathrm{C},-1.55$ $43503138,0.7726576128,2.2265204765 \backslash C,-2.8772892113,-0.7106774846,0.624$ $5989595 \backslash C,-4.0079014792,0.139682802,0.761100162 \backslash C,-3.0128891992,-1.805$ $7149254,-0.2729068438 \backslash \mathrm{C},-5.1885854731,-0.0957289025,0.0595439109 \backslash \mathrm{H},-3$. $9608088348,0.9935168688,1.4275936528 \backslash \mathrm{C},-4.1929182412,-2.0338920707,-0$. $9735118823 \backslash \mathrm{H},-2.1796042107,-2.4826480068,-0.4277195073 \backslash \mathrm{C},-5.295365182$, $-1.1830831533,-0.8145432637 \backslash \mathrm{H},-6.0320404033,0.5766776361,0.1953410821 \backslash$ $\mathrm{H},-4.2550276886,-2.8816509758,-1.6513381182 \backslash \mathrm{H},-6.215908403,-1.36416639$ $08,-1.3619694184 \backslash \mathrm{H},-2.3063580485,0.7685863332,3.0292211105 \backslash \mathrm{H},-0.570810$ $6482,0.8375339033,2.698674257 \backslash \mathrm{H},-1.7096553336,1.6968468302,1.652004210$ $4 \backslash \mathrm{C}, 4.6248417657,-0.2283872095,0.5360415558 \backslash \mathrm{C}, 5.1362441777,-1.50961262$ $65,0.3270189689 \backslash \mathrm{C}, 4.3513620546,-2.4877406005,-0.2992912972 \backslash \mathrm{C}, 3.0549994$ $605,-2.1766434732,-0.705044317 \backslash \mathrm{C}, 1.1698853428,3.1179321462,-0.04823429$ $33 \backslash \mathrm{C},-0.073571403,3.5221406378,-0.5409292213 \backslash \mathrm{C},-0.913490875,2.60385108$ $68,-1.1825563187 \backslash \mathrm{C},-0.5071319028,1.276328786,-1.3181394161 \backslash \mathrm{H}, 5.2354511$ $615,0.5357828733,1.0118328287 \backslash \mathrm{H}, 6.147916136,-1.7411218002,0.6475286912$ $\backslash \mathrm{H}, 4.7502939719,-3.483532879,-0.4686347325 \backslash \mathrm{H}, 2.4412481531,-2.931543689$ $1,-1.1909209864 \backslash \mathrm{H}, 1.8247006136,3.8334471905,0.4439245478 \backslash \mathrm{H},-0.38025800$ $79,4.5583002847,-0.4288039306 \backslash \mathrm{H},-1.8787587994,2.9192840048,-1.56675213$ $21 \backslash \mathrm{H},-1.1569399517,0.5563596035,-1.8072591044 \backslash \backslash$ Version=IA64L-G03RevD.0 $1 \backslash$ State $=1-\mathrm{A} \backslash \mathrm{HF}=-905.7631724 \backslash \mathrm{S} 2=0.175621 \backslash \mathrm{S} 2-1=0 . \backslash \mathrm{S} 2 \mathrm{~A}=0.005094 \backslash \mathrm{RMSD}=2.38$ $5 e-09 \backslash \mathrm{RMSF}=7.982 \mathrm{e}-06 \backslash \mathrm{Thermal}=0 . \backslash \mathrm{Dipole}=0.876452,0.571655,0.3734213 \backslash \mathrm{PG}=$ $\mathrm{C} 01[\mathrm{X}(\mathrm{C} 22 \mathrm{H} 21 \mathrm{~N} 1)] \backslash \backslash \mathrm{Q}$ 


\section{ACD/AMS TS1 (UB3-LYP, acetone)}

$1 \backslash 1 \backslash G I N C-A C 40 \backslash F T S \backslash U B 3 L Y P \backslash 6-31+G(d, p) \backslash C 22 H 21 N 1 \backslash B U C 562 \backslash 13-J u n-2006 \backslash 0 \backslash \backslash \#$ $\mathrm{UB} 3 \mathrm{LYP} / 6-31+\mathrm{G}(\mathrm{d}, \mathrm{p})$ Guess=(Mix,Always) Opt=(TS,CalcFC,NoEigenTest) SCRF $=($ Read, IEFPCM, Solvent $=$ Acetone $) \backslash \backslash$ PhMeC2H 2 TS C13H10NH B3Opt Acetone 02 $\backslash 0,1 \backslash \mathrm{H}, 0.2316061275,-0.981443158,0.42596 \overline{0} 40 \overline{4} 6 \backslash \mathrm{C}, 1.13 \overline{7} 56090 \overline{9} 8,-0.56 \overline{0} 235$ $2909,-0.8175411779 \backslash \mathrm{C}, 0.7215089011,0.8233307351,-0.8517043136 \backslash \mathrm{C}, 1.55763$ $82747,1.809213979,-0.25428099 \backslash \mathrm{N}, 2.7884913594,1.4192242716,0.2568667796$ $\backslash \mathrm{C}, 3.3189061683,0.1424594983,0.1133946315 \backslash \mathrm{C}, 2.5071421173,-0.8742955529$ $,-0.4588258804 \backslash \mathrm{H}, 0.6303029392,-1.2520070064,-1.4880755483 \backslash \mathrm{H}, 3.38948119$ $72,2.139555462,0.641936619 \backslash \mathrm{C},-0.5066116958,-1.2609699995,1.3748647776 \backslash$ $\mathrm{H}, 0.179970366,-1.1140923871,2.2129390971 \backslash \mathrm{H},-0.6832872451,-2.3247481305$ $, 1.1998427 \backslash \mathrm{C},-1.6611966065,-0.4116844282,1.4012448816 \backslash \mathrm{C},-1.6001209087$, $0.8672926391,2.1967417724 \backslash \mathrm{C},-2.8691795195,-0.7308919628,0.6625432469 \backslash \mathrm{C}$ $,-4.0246215518,0.0924676493,0.7486511084 \backslash \mathrm{C},-2.9654817817,-1.8713457747$ $,-0.1822243597 \backslash \mathrm{C},-5.1918098113,-0.2103600775,0.0503351485 \backslash \mathrm{H},-4.0075329$ $362,0.9788670022,1.3727939733 \backslash \mathrm{C},-4.1324428615,-2.1668689146,-0.8797109$ $233 \backslash \mathrm{H},-2.1115642611,-2.5298864933,-0.2993415524 \backslash \mathrm{C},-5.2600718386,-1.341$ $5564514,-0.7705274085 \backslash \mathrm{H},-6.0548241523,0.443686318,0.1464010331 \backslash \mathrm{H},-4.16$ $39739732,-3.0472036891,-1.5167475995 \backslash \mathrm{H},-6.1699581506,-1.5749857205,-1$. $315769611 \backslash \mathrm{H},-2.3578054727,0.883938553,2.9938974219 \backslash \mathrm{H},-0.6224394266,0.9$ $828022151,2.6712893425 \backslash \mathrm{H},-1.7755167203,1.7567951388,1.5752226121 \backslash \mathrm{C}, 4.6$ $315720123,-0.1397301488,0.518769618 \backslash \mathrm{C}, 5.152521935,-1.4246478179,0.3634$ $310041 \backslash \mathrm{C}, 4.3700603262,-2.4384410833,-0.2071700755 \backslash \mathrm{C}, 3.0660727514,-2.15$ $8887623,-0.6113021555 \backslash C, 1.1387960963,3.1439555079,-0.182507085 \backslash C,-0.11$ $30272919,3.5132012787,-0.6810358604 \backslash C,-0.950893893,2.5584014442,-1.270$ $1881308 \backslash \mathrm{C},-0.5339427075,1.229447756,-1.3472609222 \backslash \mathrm{H}, 5.2397615467,0.651$ $4778796,0.951076128 \backslash \mathrm{H}, 6.1699281266,-1.6316288962,0.6822509232 \backslash \mathrm{H}, 4.7767$ $74736,-3.4372626586,-0.3343819936 \backslash \mathrm{H}, 2.4537063553,-2.9411945318,-1.0533$ $904838 \backslash \mathrm{H}, 1.7917913971,3.8871358073,0.2689919032 \backslash \mathrm{H},-0.4282550698,4.5506$ $034332,-0.614216593 \backslash \mathrm{H},-1.9226913239,2.8472416832,-1.6586174685 \backslash \mathrm{H},-1.18$ $18264437,0.4811355455,-1.7945479935 \backslash \backslash$ Version=IA64L-G03RevD . 0 1 \State=1$\mathrm{A} \backslash \mathrm{HF}=-905.7634045 \backslash \mathrm{S} 2=0.173211 \backslash \mathrm{S} 2-1=0 . \backslash \mathrm{S} 2 \mathrm{~A}=0.004924 \backslash \mathrm{RMSD}=7.474 \mathrm{e}-09 \backslash \mathrm{RMSF}$ $=3.811 \mathrm{e}-06 \backslash \mathrm{Thermal}=0 . \backslash \mathrm{Dipole}=0.9491647,0.6425819,0.3676537 \backslash \mathrm{PG}=\mathrm{C} 01 \quad[\mathrm{X}(\mathrm{C}$ $22 \mathrm{H} 21 \mathrm{~N} 1)] \backslash \backslash$ @ 


\section{ACD/AMS TS1 (UB3-LYP, $\left.\mathrm{CH}_{3} \mathrm{CN}\right)$}

$1 \backslash 1 \backslash G I N C-A C 37 \backslash F T S \backslash U B 3 L Y P \backslash 6-31+G(d, p) \backslash C 22 H 21 N 1 \backslash B U C 562 \backslash 13-J u n-2006 \backslash 0 \backslash \backslash \#$ $\mathrm{UB} 3 \mathrm{LYP} / 6-31+\mathrm{G}(\mathrm{d}, \mathrm{p})$ Guess=(Mix,Always) Opt=(TS,CalcFC,NoEigenTest) SCRF $=($ Read, IEFPCM, Solvent $=\mathrm{CH} 3 \mathrm{CN}) \backslash \backslash \mathrm{PhMeC} 2 \mathrm{H} 2$ TS C $13 \mathrm{H} 10 \mathrm{NH}$ B3Opt_CH3CN_02 $\backslash 0,1$ $\backslash \mathrm{H}, 0.2330500867,-0.9889872154,0.417182 \overline{8} 23 \overline{7} \backslash \mathrm{C}, 1.142 \overline{3} 10234 \overline{4},-0.5 \overline{6} 3542752$ $1,-0.8224558203 \backslash \mathrm{C}, 0.7219216715,0.8186957332,-0.8564056942 \backslash \mathrm{C}, 1.55250533$ $25,1.8066178045,-0.2545276039 \backslash N, 2.7826932808,1.4202690606,0.260764374 \backslash$ C, $3.317578273,0.1453720217,0.1182054902 \backslash \mathrm{C}, 2.5114689285,-0.8734611533,-$ $0.458469534 \backslash \mathrm{H}, 0.6400179062,-1.2563219923,-1.4957274271 \backslash \mathrm{H}, 3.3797203703$, $2.1421649737,0.6497128135 \backslash \mathrm{C},-0.5056830067,-1.2714707474,1.3647508129 \backslash \mathrm{H}$ $, 0.1824912818,-1.1316636729,2.2027977389 \backslash \mathrm{H},-0.6865783931,-2.3335714953$ $, 1.1840291853 \backslash \mathrm{C},-1.6569040215,-0.4177855052,1.3970101651 \backslash \mathrm{C},-1.58776138$ $25,0.8593007039,2.1949262584 \backslash C,-2.8688829179,-0.7300369518,0.661841886$ $1 \backslash \mathrm{C},-4.0198327959,0.0991593011,0.7530730277 \backslash \mathrm{C},-2.9741002643,-1.8690003$ $425,-0.1839597397 \backslash \mathrm{C},-5.1910089459,-0.1966315108,0.0583602443 \backslash \mathrm{H},-3.9960$ $0863,0.984649417,1.3782479181 \backslash \mathrm{C},-4.144981314,-2.1573893862,-0.87788808$ $14 \backslash \mathrm{H},-2.1241689387,-2.5320641481,-0.3045561672 \backslash \mathrm{C},-5.267935315,-1.32624$ $87141,-0.7639359554 \backslash \mathrm{H},-6.0502662045,0.4617962613,0.158287085 \backslash \mathrm{H},-4.1832$ $961724,-3.0367664766,-1.5158954563 \backslash \mathrm{H},-6.1808473543,-1.5541650463,-1.30$ $64654638 \backslash \mathrm{H},-2.343415937,0.8783179099,2.993943082 \backslash \mathrm{H},-0.6083617384,0.969$ $2372994,2.6672526293 \backslash \mathrm{H},-1.7604699592,1.7507282059,1.575361095 \backslash \mathrm{C}, 4.6295$ $25035,-0.1330581194,0.5287653688 \backslash \mathrm{C}, 5.155228459,-1.4161256781,0.3741401$ $414 \backslash \mathrm{C}, 4.3784210484,-2.4318795965,-0.2008540799 \backslash \mathrm{C}, 3.0751861499,-2.15611$ $38721,-0.6100621495 \backslash \mathrm{C}, 1.1288172259,3.1399031174,-0.1826605708 \backslash \mathrm{C},-0.122$ $3991456,3.5055010359,-0.6853012478 \backslash \mathrm{C},-0.9548525796,2.5486277044,-1.278$ $9233388 \backslash \mathrm{C},-0.5330531455,1.2212499561,-1.3561534979 \backslash \mathrm{H}, 5.2332094248,0.65$ $97054038,0.9645026324 \backslash \mathrm{H}, 6.171969548,-1.6201854092,0.6969840182 \backslash \mathrm{H}, 4.788$ $8953615,-3.4292512941,-0.3274640769 \backslash \mathrm{H}, 2.4671449452,-2.9399206007,-1.05$ $5481562 \backslash \mathrm{H}, 1.777649281,3.8845809458,0.2723404911 \backslash \mathrm{H},-0.4414489438,4.5417$ $29105,-0.6182061082 \backslash \mathrm{H},-1.9262880896,2.8346633604,-1.6703498185 \backslash \mathrm{H},-1.17$ $67406491,0.4712383598,-1.8066588878 \backslash \backslash$ Version=IA64L-G03RevD.01\State=1$\mathrm{A} \backslash \mathrm{HF}=-905.7637679 \backslash \mathrm{S} 2=0.172763 \backslash \mathrm{S} 2-1=0 . \backslash \mathrm{S} 2 \mathrm{~A}=0.004893 \backslash \mathrm{RMSD}=1.706 \mathrm{e}-09 \backslash \mathrm{RMSF}$ $=4.175 e-06 \backslash$ Thermal $=0 . \backslash \mathrm{Dipole}=0.9652562,0.6509005,0.3727181 \backslash \mathrm{PG}=\mathrm{C} 01 \quad[\mathrm{X}(\mathrm{C}$ $22 \mathrm{H} 21 \mathrm{~N} 1$ ) ] \\@ 


\section{ACD/AMS TS2 (RB3-LYP, gas-phase)}

$1 \backslash 1 \backslash G I N C-A C 6 \backslash F T S \backslash R B 3 L Y P \backslash 6-31+G(d, p) \backslash C 22 H 21 N 1 \backslash B U C 562 \backslash 11-J u n-2006 \backslash 0 \backslash \backslash \# B$ $3 \mathrm{LYP} / 6-31+\mathrm{G}(\mathrm{d}, \mathrm{p})$ Opt $=(\mathrm{TS}, \mathrm{CalcFC}, \mathrm{NOE}$ igenTest $) \backslash \backslash \mathrm{PhMeC} 2 \mathrm{H} 2$ TS_C13H10NH_B3O

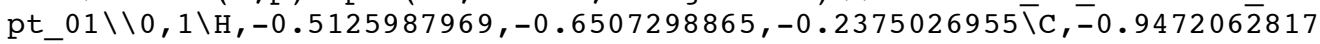
$, 0.7094397918,-1.1297753169 \backslash \mathrm{C}, 0.0711123522,1.5308469927,-0.5399304672 \backslash$ $\mathrm{C}, 1.2778564876,1.7611842504,-1.2652295652 \backslash \mathrm{N}, 1.3818443642,1.2500915386$, $-2.5524143866 \backslash \mathrm{C}, 0.3473578705,0.6122247833,-3.2284119432 \backslash \mathrm{C},-0.859221270$ $2,0.3467314401,-2.5255996846 \backslash \mathrm{H},-1.934456976,0.718586247,-0.6741974488 \backslash$ $\mathrm{H}, 2.2204071768,1.4627427873,-3.0737379233 \backslash \mathrm{C},-0.1375019818,-1.537060111$ $1,0.485446164 \backslash \mathrm{H}, 0.5674603809,-2.0648919417,-0.1627738462 \backslash \mathrm{H},-1.04587373$ $62,-2.1294969417,0.619261087 \backslash \mathrm{C}, 0.4590404081,-1.0159129168,1.6877599144$ $\backslash C, 1.9485332966,-0.8028281561,1.7212295649 \backslash \mathrm{C},-0.3271807018,-0.72501314$ $74,2.8700036103 \backslash \mathrm{C}, 0.287375126,-0.2843127012,4.0736199397 \backslash \mathrm{C},-1.74315037$ $98,-0.8604654488,2.8948296955 \backslash \mathrm{C},-0.4590823237,-0.009150721,5.216591633$ $1 \backslash \mathrm{H}, 1.3636481161,-0.1606956738,4.1131848438 \backslash \mathrm{C},-2.4830703679,-0.5807779$ $425,4.038455656 \backslash \mathrm{H},-2.269890188,-1.1842054806,2.0035387672 \backslash \mathrm{C},-1.8504935$ $402,-0.1532365595,5.2131320341 \backslash \mathrm{H}, 0.0500511821,0.3208124206,6.118329824$ $5 \backslash \mathrm{H},-3.5635559858,-0.6960102972,4.0156603874 \backslash \mathrm{H},-2.4306587875,0.0625960$ $356,6.1051990378 \backslash \mathrm{H}, 2.4257603047,-1.4316959358,2.4875439718 \backslash \mathrm{H}, 2.3994020$ $89,-1.0580815329,0.7590567921 \backslash \mathrm{H}, 2.2220743561,0.2376101263,1.9504739468$ $\backslash \mathrm{C}, 0.4863544969,0.242726267,-4.5724041002 \backslash \mathrm{C},-0.5633439061,-0.392150580$ $3,-5.2369426275 \backslash C,-1.7641509939,-0.6552367199,-4.5642601772 \backslash C,-1.90370$ $75335,-0.2871244697,-3.2284858174 \backslash \mathrm{C}, 2.3274011189,2.4807655706,-0.68720$ $27353 \backslash C, 2.2082904769,2.9663283719,0.6198024533 \backslash C, 1.0359426247,2.750667$ $0551,1.3504304539 \backslash \mathrm{C},-0.0128194379,2.0296876646,0.7776152585 \backslash \mathrm{H}, 1.415688$ $0413,0.4573781996,-5.0951728328 \backslash \mathrm{H},-0.4453785753,-0.6749552577,-6.27850$ $23555 \backslash \mathrm{H},-2.5843844792,-1.1429106974,-5.0818991573 \backslash \mathrm{H},-2.833919678,-0.48$ $84928216,-2.7032315004 \backslash \mathrm{H}, 3.2376355968,2.6566504699,-1.2558742418 \backslash \mathrm{H}, 3.0$ $317608786,3.5221562952,1.0584302425 \backslash \mathrm{H}, 0.9427842635,3.1310714853,2.3625$ $778302 \backslash \mathrm{H},-0.924718087,1.8584881483,1.3408147139 \backslash \backslash$ Version=IA64L-G03RevD $.01 \backslash$ State $=1-A \backslash H F=-905.7564563 \backslash \mathrm{RMSD}=6.214 \mathrm{e}-09 \backslash \mathrm{RMSF}=3.954 \mathrm{e}-06 \backslash \mathrm{Thermal}=0$. $\backslash$ Dipole $=0.5731342,0.0954959,-0.6049986 \backslash P G=C 01 \quad[X(C 22 \mathrm{H} 21 \mathrm{~N} 1)] \backslash \backslash @$ 
ACD/AMS TS2 (UB3-LYP, gas-phase)

$1 \backslash 1 \backslash G I N C-A C 2 \backslash F T S \backslash U B 3 L Y P \backslash 6-31+G(d, p) \backslash C 22 H 21 N 1 \backslash B U C 562 \backslash 14-J u n-2006 \backslash 0 \backslash \backslash \# U$ B3LYP $/ 6-31+G(d, p)$ Guess $=($ Mix, Always $)$ Opt $=($ TS , CalcFC,NoEigenTest $) \backslash \backslash$ PhMe C2H2_TS_C13H10NH_B3Opt_02\\0,1\H,0.2156193521,-1.009290997,0.396735571 $2 \backslash \mathrm{C}, \overline{1} .1 \overline{3} 14340923,-0.55 \overline{4} 2977048,-0.833555153 \backslash \mathrm{C}, 0.7334340663,0.834918930$ $2,-0.8151955734 \backslash \mathrm{C}, 1.5821874143,1.7859517329,-0.1824407654 \backslash \mathrm{N}, 2.80650741$ $26,1.3557628472,0.3164573885 \backslash C, 3.3233363661,0.0788701109,0.1198291764 \backslash$ $\mathrm{C}, 2.4966149887,-0.9022534742,-0.489654942 \backslash \mathrm{H}, 0.6153479329,-1.2104357767$ $,-1.5317817565 \backslash \mathrm{H}, 3.4211519795,2.052856665,0.7116179435 \backslash \mathrm{C},-0.5312334202$ $,-1.3103835696,1.3316628107 \backslash \mathrm{H}, 0.1532165433,-1.2098073378,2.1779673122 \backslash$ $\mathrm{H},-0.7299151798,-2.3630334898,1.1183754542 \backslash \mathrm{C},-1.6685480686,-0.43967762$ $19,1.384319305 \backslash \mathrm{C},-1.5876648184,0.8037831372,2.2320659448 \backslash \mathrm{C},-2.87840461$ $9,-0.705676507,0.6274043611 \backslash \mathrm{C},-4.0157847132,0.1381626626,0.7373610387 \backslash$ $C,-2.9925097942,-1.8114507534,-0.2587040289 \backslash C,-5.1832052983,-0.1125636$ $322,0.0201385644 \backslash \mathrm{H},-3.9842988995,0.9985656752,1.3960622505 \backslash \mathrm{C},-4.159433$ $6964,-2.0550893448,-0.9751156758 \backslash \mathrm{H},-2.1528879396,-2.4851227634,-0.3908$ $274098 \backslash \mathrm{C},-5.268591532,-1.209637391,-0.8432038378 \backslash \mathrm{H},-6.0331831811,0.554$ $7736931,0.1355647005 \backslash \mathrm{H},-4.2061984831,-2.910937175,-1.6433412935 \backslash \mathrm{H},-6.1$ $789613467,-1.4028121311,-1.4026381759 \backslash \mathrm{H},-2.3449209982,0.7956375326,3.0$ $296341619 \backslash \mathrm{H},-0.6084744799,0.8845972562,2.7104957236 \backslash \mathrm{H},-1.7497866226,1$. $7212624597,1.6492787493 \backslash \mathrm{C}, 4.6315999617,-0.2348201096,0.5107077578 \backslash \mathrm{C}, 5$. $1365050787,-1.5191435842,0.30501386 \backslash \mathrm{C}, 4.339791581,-2.4982629839,-0.302$ $2476274 \backslash \mathrm{C}, 3.0393733664,-2.1859720752,-0.6927807007 \backslash \mathrm{C}, 1.1847613637,3.12$ $21467493,-0.0615432337 \backslash C_{,}-0.0608204149,3.5295066874,-0.5469642774 \backslash C,-0$ $.9113376016,2.6096808077,-1.1699746388 \backslash \mathrm{C},-0.5148241177,1.2781196515,-1$ $.2951637814 \backslash \mathrm{H}, 5.2523062358,0.5299923662,0.9721201102 \backslash \mathrm{H}, 6.1513494614,-1$ $.7516323351,0.6130584611 \backslash \mathrm{H}, 4.7328244727,-3.4962979864,-0.4695472815 \backslash \mathrm{H}$, $2.4176314672,-2.9423608938,-1.1652689699 \backslash \mathrm{H}, 1.8478932924,3.8399660415,0$ $.4158994331 \backslash \mathrm{H},-0.3595775407,4.5685277139,-0.444181592 \backslash \mathrm{H},-1.8774546238$, $2.9263286194,-1.5499654329 \backslash \mathrm{H},-1.1737850397,0.5583772984,-1.7717439309 \backslash$ $\backslash$ Version=IA64L-G03RevD . $01 \backslash$ State $=1-A \backslash H F=-905.7569692 \backslash \mathrm{S} 2=0.180576 \backslash \mathrm{S} 2-1=0$ $. \backslash \mathrm{S} 2 \mathrm{~A}=0.005496 \backslash \mathrm{RMSD}=4.654 \mathrm{e}-09 \backslash \mathrm{RMSF}=3.383 \mathrm{e}-06 \backslash \mathrm{Thermal}=0 . \backslash \mathrm{Dipole}=0.59116$ $89,0.4319432,0.2953251 \backslash \mathrm{PG}=\mathrm{C} 01[\mathrm{X}(\mathrm{C} 22 \mathrm{H} 21 \mathrm{~N} 1)] \backslash \backslash @$ 


\section{ACD/AMS TS2 (UB3-LYP, heptane)}

$1 \backslash 1 \backslash G I N C-A C 31 \backslash F T S \backslash U B 3 L Y P \backslash 6-31+G(d, p) \backslash C 22 H 21 N 1 \backslash B U C 562 \backslash 17-J u n-2006 \backslash 0 \backslash \backslash \#$ $\mathrm{UB} 3 \mathrm{LYP} / 6-31+\mathrm{G}(\mathrm{d}, \mathrm{p})$ Guess=(Mix,Always) Opt=(TS,CalcFC,NoEigenTest) SCRF $=($ Read, IEFPCM, Solvent $=$ Heptane $) \backslash \backslash$ PhMeC2H3 TS_C13H9NH_B3Opt_Heptane 02 $\backslash \backslash$ $0,1 \backslash \mathrm{N}, 0.6590192227,-0.0477921933,-0.4120 \overline{2} 67 \overline{9} 04 \backslash \mathrm{C}, 0 . \overline{8} 01169 \overline{7} 451,1.3 \overline{2} 2704$ $2428,-0.2967486136 \backslash \mathrm{C}, 2.0856047283,1.9107456927,-0.0458815412 \backslash \mathrm{C}, 3.20949$ $36408,1.0685821353,0.0505320832 \backslash \mathrm{C}, 3.0809460027,-0.3189182012,-0.150644$ $2438 \backslash \mathrm{C}, 1.7767390632,-0.8615329702,-0.4019470295 \backslash \mathrm{H}, 4.1880469757,1.49599$ $55117,0.2522077576 \backslash \mathrm{H},-0.2671354763,-0.4745297574,0.1205994096 \backslash \mathrm{C},-1.374$ $6219763,-0.9541621584,1.2348365208 \backslash \mathrm{C},-2.5742211917,-0.7428426224,0.426$ $2964809 \backslash \mathrm{C},-3.3810537104,0.4192138987,0.569022384 \backslash \mathrm{C},-4.4976485815,0.642$ $8814857,-0.234876067 \backslash \mathrm{C},-4.8635839973,-0.2771789903,-1.2227739437 \backslash \mathrm{C},-4$. $0887771692,-1.4323664662,-1.3856672797 \backslash \mathrm{C},-2.9750690987,-1.6616201033,-$ $0.5831167306 \backslash C,-0.9558604214,-2.3781142493,1.5527624898 \backslash \mathrm{C},-1.102481240$ $4,-0.0000909457,2.3818927037 \backslash \mathrm{H},-3.1392282744,1.1465863295,1.3358461233$ $\backslash \mathrm{H},-5.0920263758,1.5403280347,-0.0821088746 \backslash \mathrm{H},-5.7339306212,-0.1019932$ $824,-1.8480541766 \backslash \mathrm{H},-4.3562806394,-2.1621573114,-2.1455993362 \backslash \mathrm{H},-2.401$ $0414917,-2.5679683112,-0.743907446 \backslash \mathrm{H}, 0.0856084395,-2.4170901448,1.8910$ $729657 \backslash \mathrm{H},-1.5720863342,-2.7884437914,2.3712200176 \backslash \mathrm{H},-1.0532254746,-3.0$ $684624315,0.7115430396 \backslash \mathrm{H},-1.8572771787,-0.093157727,3.1813514779 \backslash \mathrm{H},-0$. $1290640405,-0.2176701617,2.8348665771 \backslash \mathrm{H},-1.0883094883,1.0510171348,2.0$ $752547745 \backslash \mathrm{C}, 4.194481807,-1.208612954,-0.1385198952 \backslash \mathrm{C}, 4.0312607485,-2.5$ $553158374,-0.3834417623 \backslash \mathrm{C}, 2.7433420762,-3.074878411,-0.657674808 \backslash \mathrm{C}, 1.6$ $383575415,-2.2420052594,-0.6685703523 \backslash \mathrm{C},-0.3307372163,2.1558295727,-0$. $447334212 \backslash \mathrm{C},-0.2087469114,3.5294598528,-0.3294127282 \backslash \mathrm{C}, 1.0489013501,4$. $1202544814,-0.0590291794 \backslash \mathrm{C}, 2.1690024999,3.3283659652,0.0770259696 \backslash \mathrm{H}, 5$. $1802890209,-0.7996856106,0.0666313137 \backslash \mathrm{H}, 4.8903160891,-3.2194165057,-0$. $3732514075 \backslash \mathrm{H}, 2.6215212297,-4.1332673258,-0.8672743512 \backslash \mathrm{H}, 0.6529305761,-$ $2.6351849691,-0.8925209932 \backslash \mathrm{H}, 3.1395732442,3.7735548565,0.2794500656 \backslash \mathrm{H}$, $1.1298608203,5.1992024486,0.033563884 \backslash \mathrm{H},-1.0851905024,4.158544281,-0.4$ $527361315 \backslash \mathrm{H},-1.2918394096,1.7017567677,-0.6642451444 \backslash \backslash$ Version=IA64L-G0 3 RevD.01 \State $=1-\mathrm{A} \backslash \mathrm{HF}=-905.7581459 \backslash \mathrm{S} 2=0.404974 \backslash \mathrm{S} 2-1=0 . \backslash \mathrm{S} 2 \mathrm{~A}=0.030189 \backslash \mathrm{RM}$ $\mathrm{SD}=4.216 \mathrm{e}-09 \backslash \mathrm{RMSF}=1.765 \mathrm{e}-06 \backslash \mathrm{Thermal}=0 . \backslash \mathrm{Dipole}=0.9863764,0.2177507,0.07$ $19254 \backslash P G=C 01 \quad[X(C 22 \mathrm{H} 21 \mathrm{~N} 1)] \backslash \backslash @$

\section{ACD/AMS TS2 (UB3-LYP, ether)}

$1 \backslash 1 \backslash G I N C-A C 7 \backslash F T S \backslash U B 3 L Y P \backslash 6-31+G(d, p) \backslash C 22 H 21 N 1 \backslash B U C 562 \backslash 21-J u n-2006 \backslash 0 \backslash \backslash \# U$ B3LYP $/ 6-31+G(d, p)$ Guess $=($ Mix, Always $)$ Opt $=($ TS, CalcFC, NoEigenTest $)$ SCRF $=$ (Read, IEFPCM, Solvent=Ether) \\PhMeC2H3_TS_C13H9NH_B3Opt_Ether_02 $\backslash 10,1 \backslash \mathrm{N}$ $, 0.65669,-0.058813,-0.407677 \backslash C, 0.8334 \overline{7}, 1.30935,-\overline{0} .3105 \overline{9} \backslash \mathrm{C}, 2 . \overline{1} 33143,1.8$ $68005,-0.07125 \backslash \mathrm{C}, 3.235985,0.999309,0.034228 \backslash \mathrm{C}, 3.071674,-0.387268,-0.14$ $7251 \backslash \mathrm{C}, 1.753743,-0.900775,-0.38846 \backslash \mathrm{H}, 4.225717,1.404697,0.227989 \backslash \mathrm{H},-0.2$ $77757,-0.454465,0.129184 \backslash \mathrm{C},-1.39993,-0.893527,1.255554 \backslash \mathrm{C},-2.591914,-0$. $683282,0.437108 \backslash \mathrm{C},-3.375686,0.499402,0.540588 \backslash \mathrm{C},-4.484713,0.72145,-0.2$ $74511 \backslash C,-4.867316,-0.221199,-1.234998 \backslash C,-4.116281,-1.397323,-1.358402 \backslash$ $C,-3.00976,-1.624879,-0.544927 \backslash C,-1.000491,-2.315122,1.606711 \backslash \mathrm{C},-1.116$ $978,0.083295,2.380631 \backslash \mathrm{H},-3.121491,1.245995,1.284805 \backslash \mathrm{H},-5.059824,1.6362$ $66,-0.152351 \backslash \mathrm{H},-5.731902,-0.047213,-1.868986 \backslash \mathrm{H},-4.3969,-2.14528,-2.096$ $023 \backslash \mathrm{H},-2.455264,-2.54826,-0.67549 \backslash \mathrm{H}, 0.039438,-2.358946,1.949425 \backslash \mathrm{H},-1.6$ $23785,-2.699445,2.432738 \backslash \mathrm{H},-1.103027,-3.023149,0.780969 \backslash \mathrm{H},-1.875177,0$. $019607,3.180011 \backslash \mathrm{H},-0.147828,-0.137487,2.8414 \backslash \mathrm{H},-1.087428,1.126812,2.05$ $0142 \backslash \mathrm{C}, 4.163366,-1.303902,-0.125114 \backslash \mathrm{C}, 3.966019,-2.649484,-0.351446 \backslash \mathrm{C}, 2$ $.664597,-3.140681,-0.61584 \backslash C, 1.580216,-2.280961,-0.635668 \backslash C,-0.277532$, $2.168965,-0.468308 \backslash C,-0.120497,3.540746,-0.36959 \backslash C, 1.15301,4.103134,-0$ $.111445 \backslash \mathrm{C}, 2.253278,3.284849,0.032108 \backslash \mathrm{H}, 5.159592,-0.916366,0.072271 \backslash \mathrm{H}, 4$ $.808414,-3.334848,-0.334136 \backslash \mathrm{H}, 2.515946,-4.198757,-0.810523 \backslash \mathrm{H}, 0.585122$, $-2.653981,-0.851937 \backslash \mathrm{H}, 3.235831,3.707695,0.225639 \backslash \mathrm{H}, 1.261453,5.181091$,$0.033833 \backslash \mathrm{H},-0.981335,4.190522,-0.497848 \backslash \mathrm{H},-1.25105,1.73659,-0.674475 \backslash \backslash$ Version=IA64L-G03RevD. $01 \backslash$ State $=1-A \backslash H F=-905.7605446 \backslash \mathrm{S} 2=0.39933 \backslash \mathrm{S} 2-1=0 . \backslash$ $\mathrm{S} 2 \mathrm{~A}=0.029274 \backslash \mathrm{RMSD}=4.805 \mathrm{e}-09 \backslash \mathrm{RMSF}=3.053 \mathrm{e}-06 \backslash \mathrm{Thermal}=0 . \backslash \mathrm{Dipole}=1.1691226$ $, 0.2306377,0.0811852 \backslash$ Polar $=993.5396915,99.8138166,490.9243908,-75.8053$ $371,-14.5588792,251.3332326 \backslash \mathrm{PG}=\mathrm{C} 01 \quad[\mathrm{X}(\mathrm{C} 22 \mathrm{H} 21 \mathrm{~N} 1)] \backslash \backslash @$ 


\section{ACD/AMS TS2 (UB3-LYP, acetone)}

$1 \backslash 1 \backslash G I N C-A C 39 \backslash F T S \backslash U B 3 L Y P \backslash 6-31+G(d, p) \backslash C 22 H 21 N 1 \backslash B U C 562 \backslash 17-J u n-2006 \backslash 0 \backslash \backslash \#$ $\mathrm{UB} 3 \mathrm{LYP} / 6-31+\mathrm{G}(\mathrm{d}, \mathrm{p})$ Guess=(Mix,Always) Opt=(TS,CalcFC,NoEigenTest) SCRF $=($ Read, IEFPCM, Solvent $=$ Acetone $) \backslash \backslash$ PhMeC2H3_TS_C13H9NH_B3Opt_Acetone_02 $\backslash \backslash$ $0,1 \backslash \mathrm{N}, 0.6513874772,-0.0251658503,-0.3988 \overline{3} 63 \overline{6} 28 \backslash \mathrm{C}, 0 . \overline{8} 10336 \overline{5} 911,1.3 \overline{4} 2571$ $8936,-0.2707164116 \backslash \mathrm{C}, 2.1051673412,1.9134672813,-0.0340258037 \backslash \mathrm{C}, 3.22090$ $25541,1.0578117292,0.0374810834 \backslash \mathrm{C}, 3.0732369721,-0.3262304763,-0.174874$ $6094 \backslash \mathrm{C}, 1.7596403679,-0.8524440992,-0.4126891804 \backslash \mathrm{H}, 4.2072480728,1.47208$ $82444,0.2290567301 \backslash \mathrm{H},-0.2722566271,-0.4465679761,0.1339556768 \backslash \mathrm{C},-1.385$ $6352403,-0.9320710322,1.2549810783 \backslash C,-2.5758527677,-0.755249803,0.4272$ $589702 \backslash \mathrm{C},-3.3949437555,0.4041892837,0.5262969338 \backslash \mathrm{C},-4.5019474785,0.596$ $7092407,-0.2989972364 \backslash \mathrm{C},-4.8483685871,-0.3536646741,-1.2657062466 \backslash \mathrm{C},-4$ $.0625286547,-1.5074077195,-1.3848254641 \backslash \mathrm{C},-2.9574465556,-1.7054938343$, $-0.5616530588 \backslash \mathrm{C},-0.9380301031,-2.3411160552,1.5980319726 \backslash \mathrm{C},-1.14469033$ $53,0.0448754816,2.3896440823 \backslash \mathrm{H},-3.1700136663,1.1559028885,1.2748245462$ $\backslash \mathrm{H},-5.1036834096,1.4945727684,-0.180130241 \backslash \mathrm{H},-5.7114808604,-0.20265358$ $22,-1.9073806931 \backslash \mathrm{H},-4.314493485,-2.2611007721,-2.1268355456 \backslash \mathrm{H},-2.37567$ $42206,-2.6122193147,-0.6897738586 \backslash \mathrm{H}, 0.103091715,-2.3513440191,1.940084$ $8329 \backslash \mathrm{H},-1.5467626597,-2.7515484675,2.422413972 \backslash \mathrm{H},-1.01597759,-3.047750$ $2383,0.7686740962 \backslash \mathrm{H},-1.9056877465,-0.0521405495,3.1830645241 \backslash \mathrm{H},-0.1718$ $204933,-0.1458022569,2.8559738261 \backslash \mathrm{H},-1.1494067829,1.0916944431,2.06877$ $76541 \backslash \mathrm{C}, 4.1777677579,-1.2276693086,-0.1875735433 \backslash \mathrm{C}, 3.9960733127,-2.569$ $9886047,-0.4439655332 \backslash \mathrm{C}, 2.6983346484,-3.0729955617,-0.704834179 \backslash \mathrm{C}, 1.60$ $18824865,-2.2286252778,-0.691125749 \backslash \mathrm{C},-0.3142270171,2.1901467233,-0.39$ $40224984 \backslash \mathrm{C},-0.1745553983,3.5612652065,-0.2647726359 \backslash \mathrm{C}, 1.0944663007,4.1$ $350861949,-0.0092403194 \backslash \mathrm{C}, 2.2074914865,3.3290750902,0.1013818319 \backslash \mathrm{H}, 5.1$ $707476604,-0.8310290186,0.0073053347 \backslash \mathrm{H}, 4.8477695911,-3.2436913332,-0.4$ $530655407 \backslash \mathrm{H}, 2.561926698,-4.1280211925,-0.9229299404 \backslash \mathrm{H}, 0.6095825356,-2$. $6107772952,-0.9043819816 \backslash \mathrm{H}, 3.1864604625,3.7607116675,0.2929012313 \backslash \mathrm{H}, 1$. $1891388126,5.2121589234,0.092745495 \backslash \mathrm{H},-1.0456510831,4.2018647859,-0.36$ $61176017 \backslash \mathrm{H},-1.2844903266,1.749140467,-0.5977666374 \backslash \backslash$ Version=IA64L-G03R evD. $01 \backslash$ State $=1-\mathrm{A} \backslash \mathrm{HF}=-905.7607908 \backslash \mathrm{S} 2=0.395104 \backslash \mathrm{S} 2-1=0 . \backslash \mathrm{S} 2 \mathrm{~A}=0.028557 \backslash \mathrm{RMSD}$ $=5.791 \mathrm{e}-09 \backslash \mathrm{RMSF}=2.454 \mathrm{e}-06 \backslash \mathrm{Thermal}=0 . \backslash \mathrm{Dipole}=1.2657029,0.2762243,0.0926$ $872 \backslash \mathrm{PG}=\mathrm{C} 01 \quad[\mathrm{X}(\mathrm{C} 22 \mathrm{H} 21 \mathrm{~N} 1)] \backslash \backslash @$

\section{ACD/AMS TS2 (UB3-LYP, $\mathrm{CH}_{3} \mathrm{CN}$ )}

$1 \backslash 1 \backslash G I N C-A C 2 \backslash F T S \backslash U B 3 L Y P \backslash 6-31+G(d, p) \backslash C 22 H 21 N 1 \backslash B U C 562 \backslash 21-J u n-2006 \backslash 0 \backslash \backslash \# U$ B3LYP $/ 6-31+G(d, p)$ Guess $=($ Mix, Always $)$ Opt $=($ TS, CalcFC, NoEigenTest $) \quad S C R F=$

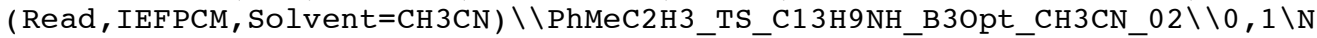
$, 0.648725,-0.054838,-0.391024 \backslash \mathrm{C}, 0.862 \overline{9} 36,1.30865 \overline{1},-0.3 \overline{0} 4519 \backslash \bar{C}, 2.179964$ $, 1.833626,-0.084597 \backslash \mathrm{C}, 3.260208,0.936076,0.013796 \backslash \mathrm{C}, 3.056497,-0.446702$, $-0.156292 \backslash \mathrm{C}, 1.722578,-0.926403,-0.378942 \backslash \mathrm{H}, 4.26263,1.315735,0.19333 \backslash \mathrm{H}$, $-0.290958,-0.42217,0.152915 \backslash C,-1.424901,-0.829361,1.285428 \backslash C,-2.603713$ $,-0.640747,0.444383 \backslash \mathrm{C},-3.38134,0.550048,0.495896 \backslash \mathrm{C},-4.476299,0.751742$, $-0.343181 \backslash \mathrm{C},-4.851501,-0.220216,-1.277194 \backslash \mathrm{C},-4.106813,-1.404796,-1.349$ $33 \backslash \mathrm{C},-3.013897,-1.612054,-0.512231 \backslash \mathrm{C},-1.027189,-2.239869,1.680011 \backslash \mathrm{C},-1$ $.155755,0.177955,2.386684 \backslash \mathrm{H},-3.133806,1.320153,1.218175 \backslash \mathrm{H},-5.045995,1$. $674272,-0.260846 \backslash \mathrm{H},-5.705268,-0.061981,-1.929554 \backslash \mathrm{H},-4.381688,-2.175592$ $,-2.065193 \backslash \mathrm{H},-2.464602,-2.543166,-0.603544 \backslash \mathrm{H}, 0.012046,-2.273646,2.0263$ $79 \backslash \mathrm{H},-1.65188,-2.599511,2.516115 \backslash \mathrm{H},-1.127021,-2.972558,0.876046 \backslash \mathrm{H},-1.9$ $2326,0.134619,3.178606 \backslash \mathrm{H},-0.192153,-0.029357,2.865023 \backslash \mathrm{H},-1.123196,1.21$ $2508,2.029786 \backslash \mathrm{C}, 4.123696,-1.392045,-0.140694 \backslash \mathrm{C}, 3.887708,-2.73309,-0.35$ $6355 \backslash \mathrm{C}, 2.57055,-3.190908,-0.602955 \backslash \mathrm{C}, 1.50912,-2.302871,-0.615612 \backslash \mathrm{C},-0$. $226472,2.196915,-0.454177 \backslash \mathrm{C},-0.031316,3.56458,-0.366878 \backslash \mathrm{C}, 1.260112,4.0$ $9409,-0.128365 \backslash \mathrm{C}, 2.33967,3.247421,0.007502 \backslash \mathrm{H}, 5.131992,-1.03012,0.04280$ $6 \backslash \mathrm{H}, 4.711444,-3.440673,-0.344332 \backslash \mathrm{H}, 2.391468,-4.245691,-0.78904 \backslash \mathrm{H}, 0.502$ $111,-2.651021,-0.817909 \backslash \mathrm{H}, 3.335464,3.644667,0.186304 \backslash \mathrm{H}, 1.398373,5.1690$ $66,-0.059308 \backslash \mathrm{H},-0.875843,4.236549,-0.48812 \backslash \mathrm{H},-1.213991,1.789531,-0.644$ $738 \backslash \backslash$ Version=IA64L-G03RevD.01 $\backslash$ State $=1-A \backslash H F=-905.7609977 \backslash S 2=0.394594 \backslash S 2$ $-1=0 . \backslash \mathrm{S} 2 \mathrm{~A}=0.02848 \backslash \mathrm{RMSD}=9.162 \mathrm{e}-09 \backslash \mathrm{RMSF}=2.357 \mathrm{e}-06 \backslash \mathrm{Thermal}=0 . \backslash \mathrm{Dipole}=1.29$ $59454,0.234937,0.0923887 \backslash$ Polar $=1160.1727071,123.025785,531.3961561,-10$ $2.7732742,-19.6011373,277.1514093 \backslash \mathrm{PG}=\mathrm{C} 01 \quad[\mathrm{X}(\mathrm{C} 22 \mathrm{H} 21 \mathrm{~N} 1)] \backslash \backslash @$ 


\section{The $C H D / D D Q$ reaction}

CHD/DDQ TS1 (RB3-LYP, gas phase)

$1 \backslash 1 \backslash G I N C-A C 22 \backslash F T S \backslash R B 3 L Y P \backslash 6-31+G(d, p) \backslash C 14 H 8 C 12 N 202 \backslash B U C 562 \backslash 13-J u 1-2006 \backslash 0$ $\backslash \backslash \#$ B3LYP/6-31+G(d,p) Opt=(TS,CalcFC, NoEigenTest) \\DDQ_TS_C6H8_B3Opt_0 $1 \backslash \backslash 0,1 \backslash C,-0.6920699815,0.7411872156,1.4011947596 \backslash C,-1.951 \overline{7} 9373 \overline{3} 5,0.2 \overline{2} 4$ $3052129,1.9116703093 \backslash \mathrm{C},-2.1165020319,-1.1074938199,2.1820203561 \backslash \mathrm{C},-1.0$ $281189055,-2.0918644835,1.9610269349 \backslash \mathrm{C}, 0.2645321776,-1.5302835789,1.50$ $15292958 \backslash \mathrm{C}, 0.4174965251,-0.1787917685,1.2510759965 \backslash \mathrm{Cl},-3.210443493,1.3$ $817124769,2.2035566526 \backslash \mathrm{Cl},-3.5931224157,-1.7496841987,2.7968700059 \backslash \mathrm{C}, 1$ $.3460003244,-2.4454700288,1.3393279124 \backslash \mathrm{N}, 2.228463888,-3.1884559559,1.1$ $843015004 \backslash \mathrm{C}, 1.6892302254,0.3701034479,0.880964713 \backslash \mathrm{N}, 2.7261296208,0.814$ $4727317,0.5994512606 \backslash 0,-1.173696864,-3.2925903835,2.1661594742 \backslash 0,-0.54$ $28852071,1.987800267,1.1143721392 \backslash \mathrm{H},-0.7761981735,2.2418152252,-0.0394$ $375689 \backslash \mathrm{C},-1.0701905823,2.2129150214,-1.3875610645 \backslash \mathrm{C}, 0.0305870029,1.388$ $4359249,-1.8493466178 \backslash \mathrm{C},-0.1114518269,0.0467413512,-2.0094120116 \backslash \mathrm{C},-1$. $4180510948,-0.6522185176,-1.8247411862 \backslash C,-2.5520263198,0.2652038323,-1$ $.5005817506 \backslash \mathrm{C},-2.3879907103,1.6034762485,-1.3460870728 \backslash \mathrm{H},-1.0103943461$ $, 3.2877956488,-1.5638832867 \backslash \mathrm{H}, 1.0018389622,1.8514860257,-1.9949400462 \backslash$ $\mathrm{H}, 0.7435661706,-0.5544221419,-2.3074109232 \backslash \mathrm{H},-1.6579538899,-1.25447058$ $92,-2.7177360853 \backslash \mathrm{H},-3.5435767247,-0.1718438078,-1.4159619486 \backslash \mathrm{H},-3.2434$ $454205,2.2326344795,-1.1192524843 \backslash \mathrm{H},-1.3182071763,-1.4253698352,-1.032$ $8762635 \backslash \backslash$ Version=IA64L-G03RevD.01 \State $=1-A \backslash H F=-1718.5333574 \backslash$ RMSD $=9.27$ $8 \mathrm{e}-09 \backslash \mathrm{RMSF}=7.067 \mathrm{e}-06 \backslash \mathrm{Thermal}=0 . \backslash \mathrm{Dipole}=-2.1008724,1.8593816,-1.9712638$ $\backslash \mathrm{PG}=\mathrm{C} 01 \quad[\mathrm{X}(\mathrm{C} 14 \mathrm{H} 8 \mathrm{C} 12 \mathrm{~N} 2 \mathrm{O} 2)] \backslash \backslash @$

\section{CHD/DDQ TS1 (UB3-LYP, gas phase)}

$1 \backslash 1 \backslash G I N C-A C 22 \backslash F T S \backslash U B 3 L Y P \backslash 6-31+G(d, p) \backslash C 14$ H $8 C 12 N 202 \backslash B U C 562 \backslash 14-J u 1-2006 \backslash 0$ $\backslash \backslash \#$ UB3LYP $/ 6-31+G(d, p)$ Guess=(Mix,Always ) Opt=(TS, CalcFC, NoEigenTest $) \backslash$

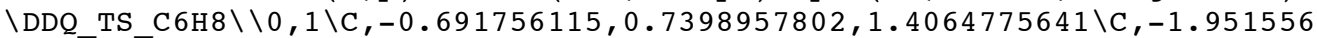
$8002,0 . \overline{2} 239959599,1.9177316989 \backslash \mathrm{C},-2.1160137679,-1.1071619421,2.1918579$ $338 \backslash \mathrm{C},-1.0273543992,-2.0914389734,1.9733567735 \backslash \mathrm{C}, 0.2651965265,-1.53122$ $35528,1.5123804927 \backslash \mathrm{C}, 0.4176443265,-0.1804268941,1.2576672587 \backslash \mathrm{Cl},-3.210$ $7631994,1.3817413612,2.2052234893 \backslash \mathrm{Cl},-3.592523417,-1.7481964083,2.8079$ $496077 \backslash \mathrm{C}, 1.3464766879,-2.4469601511,1.352169738 \backslash \mathrm{N}, 2.2287928978,-3.1905$ $714333,1.1991934772 \backslash \mathrm{C}, 1.6885464125,0.3672782622,0.8832426686 \backslash \mathrm{N}, 2.72485$ $92652,0.8106242133,0.5978752897 \backslash 0,-1.1727440849,-3.2919845523,2.180850$ $5504 \backslash 0,-0.5427347334,1.9856833335,1.1168059353 \backslash \mathrm{H},-0.7771619055,2.23819$ $97137,-0.0402236357 \backslash \mathrm{C},-1.0707219782,2.2124706017,-1.38514678 \backslash \mathrm{C}, 0.03047$ $38236,1.391004402,-1.8529756513 \backslash \mathrm{C},-0.1111446158,0.050636128,-2.0224890$ $439 \backslash \mathrm{C},-1.4175409009,-0.6502106305,-1.8410447779 \backslash \mathrm{C},-2.5521410263,0.2647$ $777769,-1.5109768331 \backslash \mathrm{C},-2.3885559331,1.6018645757,-1.3475475207 \backslash \mathrm{H},-1.0$ $123010931,3.2880086467,-1.5585317483 \backslash \mathrm{H}, 1.0014215467,1.8555210232,-1.99$ $60254185 \backslash \mathrm{H}, 0.7438055,-0.5481775404,-2.3253805779 \backslash \mathrm{H},-1.6576740095,-1.24$ $66783786,-2.7378322514 \backslash \mathrm{H},-3.5435426458,-0.1732379057,-1.4295505201 \backslash \mathrm{H},-$ $3.24424826,2.2292902693,-1.1167734931 \backslash \mathrm{H},-1.3170121018,-1.4275976847,-1$ $.053991226 \backslash \backslash$ Version=IA6 4L-G03RevD. $01 \backslash$ State $=1-A \backslash H F=-1718.5333645 \backslash \mathrm{S} 2=0.0$ $30968 \backslash \mathrm{S} 2-1=0 . \backslash \mathrm{S} 2 \mathrm{~A}=0.000177 \backslash \mathrm{RMSD}=9.745 \mathrm{e}-09 \backslash \mathrm{RMSF}=9.361 \mathrm{e}-06 \backslash \mathrm{Thermal}=0 . \backslash \mathrm{Di}$ pole $=-2.0988005,1.842962,-1.965441 \backslash \mathrm{PG}=\mathrm{C} 01 \quad[\mathrm{X}(\mathrm{C} 14 \mathrm{H} 8 \mathrm{Cl} 2 \mathrm{~N} 2 \mathrm{O} 2)] \backslash \backslash @$ 


\section{CHD/DDQ TS1 (UB3-LYP, heptane)}

$1 \backslash 1 \backslash G I N C-A C 34 \backslash F T S \backslash U B 3 L Y P \backslash 6-31+G(d, p) \backslash C 14$ H $8 C 12 N 202 \backslash B U C 562 \backslash 27-J u 1-2006 \backslash 0$ $\backslash \backslash \#$ UB3LYP $/ 6-31+G(d, p)$ Guess=(Mix,Always) Opt=(TS,CalcFC,NoEigenTest) $\mathrm{SCRF}=($ Read, IEFPCM, Solvent $=$ Heptane $) \backslash \backslash \mathrm{DDQ}$ TS_C6H8_B30pt_Heptane_02 $\backslash \backslash 0,1 \backslash$ $C, 0.2473217608,-0.2433318882,-1.1319452103 \backslash \mathrm{C},-1.0394068898,-0.78930184$ $94,-0.7261639695 \backslash \mathrm{C},-1.9529845789,-0.0292751241,-0.0465471842 \backslash \mathrm{C},-1.6814$ $647697,1.3812394119,0.3186526609 \backslash \mathrm{C},-0.3930981927,1.9324303862,-0.15981$ $04628 \backslash \mathrm{C}, 0.5135433724,1.1546014123,-0.8571585767 \backslash \mathrm{Cl},-1.3740982883,-2.42$ $51295864,-1.192591108 \backslash \mathrm{Cl},-3.4896140035,-0.6459428206,0.4361636158 \backslash \mathrm{C},-0$ $.1363129979,3.3071655901,0.1189571321 \backslash \mathrm{N}, 0.0861638431,4.4244415993,0.35$ $78184378 \backslash \mathrm{C}, 1.7200226834,1.7182489735,-1.3880397889 \backslash \mathrm{N}, 2.6829480804,2.19$ $08978252,-1.836644443 \backslash 0,-2.4795817925,2.0656090975,0.9542414348 \backslash 0,1.12$ $10873923,-0.9600058449,-1.7439393165 \backslash \mathrm{H}, 1.9205464828,-1.5018721515,-0.9$ $794162685 \backslash C, 2.6498749213,-1.9135254226,0.0824255852 \backslash C, 3.1762993223,-0$. $6350521327,0.5275448254 \backslash \mathrm{C}, 2.5712441555,0.0714065125,1.5181997242 \backslash \mathrm{C}, 1.4$ $033060683,-0.4558318496,2.283049007 \backslash \mathrm{C}, 0.9945982975,-1.8369791142,1.888$ $9764186 \backslash \mathrm{C}, 1.6116069906,-2.526039512,0.89588605 \backslash \mathrm{H}, 3.3249526092,-2.57679$ $83794,-0.4623387939 \backslash \mathrm{H}, 4.0295336348,-0.2144893784,0.0036539926 \backslash \mathrm{H}, 2.9601$ $054145,1.046270318,1.8014685572 \backslash \mathrm{H}, 1.6096843303,-0.415023691,3.36686553$ $21 \backslash \mathrm{H}, 0.1914852008,-2.3034339915,2.4538763904 \backslash \mathrm{H}, 1.2899230816,-3.5341234$ $892,0.6518089185 \backslash \mathrm{H}, 0.5431448714,0.2391560991,2.1789598397 \backslash \backslash$ Version=IA6 $4 \mathrm{~L}-\mathrm{G} 03$ RevD $.01 \backslash$ State $=1-\mathrm{A} \backslash \mathrm{HF}=-1718.539337 \backslash \mathrm{S} 2=0 . \backslash \mathrm{S} 2-1=0 . \backslash \mathrm{S} 2 \mathrm{~A}=0 . \backslash \mathrm{RMSD}=9.65$ $8 \mathrm{e}-09 \backslash \mathrm{RMSF}=7.858 \mathrm{e}-06 \backslash \mathrm{Thermal}=0 . \backslash \mathrm{Dipole}=1.3177447,-3.4994606,1.1667504 \backslash$ $\mathrm{PG}=\mathrm{C} 01[\mathrm{X}(\mathrm{C} 14 \mathrm{H} 8 \mathrm{C} 12 \mathrm{~N} 2 \mathrm{O} 2)] \backslash \backslash @$

\section{CHD/DDQ TS1 (UB3-LYP, ether)}

$1 \backslash 1 \backslash G I N C-A C 30 \backslash F T S \backslash U B 3 L Y P \backslash 6-31+G(d, p) \backslash C 14 H 8 C l 2 N 202 \backslash B U C 562 \backslash 27-J u l-2006 \backslash 0$ $\backslash \backslash \#$ UB3LYP $/ 6-31+G(d, p)$ Guess=(Mix,Always) Opt=(TS,CalcFC,NoEigenTest) $\mathrm{SCRF}=($ Read, IEFPCM, Solvent $=$ Ether $) \backslash \backslash$ DDQ_TS_C6H8_B3Opt_Ether_02 $\backslash \backslash 0,1 \backslash \mathrm{C}, 0$. $2516821904,-0.2412639099,-1.1420585395 \backslash C_{,}-1.0354852831,-0.7862646311$,$0.7306551565 \backslash \mathrm{C},-1.9460110546,-0.0264579413,-0.0467219843 \backslash \mathrm{C},-1.67509397$ $1,1.3818356497,0.3171187011 \backslash \mathrm{C},-0.3896273385,1.9323061339,-0.1626478882$ $\backslash \mathrm{C}, 0.5180549091,1.1555877856,-0.860768431 \backslash \mathrm{Cl},-1.3750347397,-2.41849560$ $15,-1.2022898463 \backslash \mathrm{Cl},-3.4825891598,-0.6452838796,0.4388453322 \backslash \mathrm{C},-0.1372$ $689203,3.3088812208,0.1099740044 \backslash \mathrm{N}, 0.0774547646,4.429277425,0.34153655$ $74 \backslash \mathrm{C}, 1.7173130849,1.7257773184,-1.4001912879 \backslash \mathrm{N}, 2.6688910176,2.21130626$ $92,-1.8591760946 \backslash 0,-2.474371107,2.0695698833,0.9524560008 \backslash 0,1.12005709$ $82,-0.9533783695,-1.7596467396 \backslash \mathrm{H}, 1.9394275495,-1.5193023716,-0.9688200$ $423 \backslash \mathrm{C}, 2.6399754797,-1.9107584699,0.0784169251 \backslash \mathrm{C}, 3.1663534808,-0.631116$ $152,0.5269307225 \backslash C, 2.5650501019,0.0703058282,1.5243188682 \backslash C, 1.40711828$ $27,-0.465394513,2.2955015945 \backslash \mathrm{C}, 0.9916543353,-1.8410360172,1.8948180489$ $\backslash \mathrm{C}, 1.6021699778,-2.5259830282,0.8943239126 \backslash \mathrm{H}, 3.3255307085,-2.577921889$ $4,-0.4532309577 \backslash \mathrm{H}, 4.01971829,-0.210177278,0.0024751775 \backslash \mathrm{H}, 2.9565366898$, $1.0428368461,1.8142204175 \backslash \mathrm{H}, 1.6296525545,-0.4375306799,3.3787035038 \backslash \mathrm{H}$, $0.193160268,-2.3102686466,2.4648890915 \backslash \mathrm{H}, 1.2801346395,-3.5336779503,0$. $6471825416 \backslash \mathrm{H}, 0.5463771512,0.2319379689,2.2184485683 \backslash \backslash$ Version=IA64L-G03 RevD. $01 \backslash$ State $=1-\mathrm{A} \backslash \mathrm{HF}=-1718.5478256 \backslash \mathrm{S} 2=0 . \backslash \mathrm{S} 2-1=0 . \backslash \mathrm{S} 2 \mathrm{~A}=0 . \backslash \mathrm{RMSD}=4.231 \mathrm{e}-09$ $\backslash \mathrm{RMSF}=6.836 \mathrm{e}-06 \backslash \mathrm{Thermal}=0 . \backslash \mathrm{Dipole}=1.5824971,-3.9743384,1.3572659 \backslash \mathrm{PG}=\mathrm{C} 0$ $1[\mathrm{X}(\mathrm{C} 14 \mathrm{H} 8 \mathrm{Cl} 2 \mathrm{~N} 2 \mathrm{O} 2)] \backslash \backslash @$ 


\section{CHD/DDQ TS1 (UB3-LYP, acetone)}

$1 \backslash 1 \backslash G I N C-A C 27 \backslash F T S \backslash U B 3 L Y P \backslash 6-31+G(d, p) \backslash C 14 H 8 C 12 N 202 \backslash B U C 562 \backslash 27-J u 1-2006 \backslash 0$ $\backslash \backslash \#$ UB3LYP $/ 6-31+G(d, p)$ Guess=(Mix,Always) Opt=(TS,CalcFC,NoEigenTest) $\mathrm{SCRF}=($ Read, IEFPCM, Solvent $=$ Acetone $) \backslash \backslash \mathrm{DDQ}$ TS C6H8 B3Opt Acetone $02 \backslash \backslash 0,1 \backslash$ $C, 0.2534946688,-0.23881262,-1.143454276 \overline{7} \backslash \mathrm{C},-1.0 \overline{3} 29512 \overline{9} 88,-0.7 \overline{8} 38239901$ $,-0.7263627487 \backslash \mathrm{C},-1.9425484184,-0.0226302441,-0.0423194901 \backslash \mathrm{C},-1.674505$ $8826,1.387340676,0.3129530822 \backslash C,-0.3890776292,1.9361005997,-0.16659799$ $87 \backslash \mathrm{C}, 0.5196032694,1.1584193806,-0.8626378533 \backslash \mathrm{Cl},-1.373219533,-2.415974$ $3748,-1.1946113246 \backslash \mathrm{Cl},-3.4779170518,-0.6419486744,0.4479847576 \backslash \mathrm{C},-0.14$ $06750628,3.3146643316,0.0992089044 \backslash \mathrm{N}, 0.0673172495,4.4379119972,0.32366$ $9842 \backslash \mathrm{C}, 1.714999621,1.7316091355,-1.4071202733 \backslash \mathrm{N}, 2.6596492456,2.2252593$ $305,-1.8719079722 \backslash 0,-2.4771156664,2.0802547156,0.9401535216 \backslash 0,1.118740$ $1352,-0.9487445162,-1.764695845 \backslash \mathrm{H}, 1.9468447314,-1.5255728909,-0.965190$ $0704 \backslash \mathrm{C}, 2.637962402,-1.9103534893,0.076296698 \backslash \mathrm{C}, 3.1637677309,-0.6311884$ $481,0.5293066005 \backslash \mathrm{C}, 2.5635245437,0.06552806,1.53074456 \backslash \mathrm{C}, 1.4089496685,-$ $0.475582979,2.3023322798 \backslash \mathrm{C}, 0.9921839549,-1.8486537065,1.8958427076 \backslash \mathrm{C}, 1$ $.6007324461,-2.5292716642,0.891267787 \backslash \mathrm{H}, 3.3278652571,-2.5770759757,-0$. $449602916 \backslash \mathrm{H}, 4.0171705664,-0.208722714,0.0064823543 \backslash \mathrm{H}, 2.9558653366,1.03$ $58630644,1.8260781246 \backslash \mathrm{H}, 1.6365191578,-0.4543614717,3.3840131236 \backslash \mathrm{H}, 0.19$ $62163719,-2.3208826545,2.4666125243 \backslash \mathrm{H}, 1.2794695409,-3.5361419941,0.640$ $7445444 \backslash \mathrm{H}, 0.5479656451,0.2221021164,2.2347623569 \backslash \backslash$ Version=IA64L-G03Rev D. $01 \backslash$ State $=1-\mathrm{A} \backslash \mathrm{HF}=-1718.5498059 \backslash \mathrm{S} 2=0 . \backslash \mathrm{S} 2-1=0 . \backslash \mathrm{S} 2 \mathrm{~A}=0 . \backslash \mathrm{RMSD}=5.724 \mathrm{e}-09 \backslash \mathrm{RM}$ $\mathrm{SF}=8.824 \mathrm{e}-06 \backslash \mathrm{Thermal}=0 . \backslash \mathrm{Dipole}=1.6827877,-4.2060198,1.4470153 \backslash \mathrm{PG}=\mathrm{C} 01$ [ $\mathrm{X}(\mathrm{C} 14 \mathrm{H} 8 \mathrm{Cl} 2 \mathrm{~N} 2 \mathrm{O} 2)] \backslash \backslash @$

\section{CHD/DDQ TS1 (UB3-LYP, $\mathrm{CH}_{3} \mathrm{CN}$ )}

$1 \backslash 1 \backslash G I N C-A C 28 \backslash F T S \backslash U B 3 L Y P \backslash 6-31+G(d, p) \backslash C 14 H 8 C 12 N 202 \backslash B U C 562 \backslash 27-J u 1-2006 \backslash 0$ $\backslash \backslash \#$ UB3LYP $/ 6-31+G(d, p)$ Guess=(Mix,Always) Opt=(TS,CalcFC,NoEigenTest) $\mathrm{SCRF}=($ Read, IEFPCM, Solvent $=\mathrm{CH} 3 \mathrm{CN}) \backslash \backslash \mathrm{DDQ}$ TS_C6H8_B3Opt_CH3CN_02 $\backslash \backslash 0,1 \backslash \mathrm{C}, 0$. $2543649511,-0.238386983,-1.1441796436 \backslash C,-1.03 \overline{1} 92957 \overline{6} 6,-0 . \overline{7} 842371885,-0$ $.7269513826 \backslash \mathrm{C},-1.941434563,-0.0241617897,-0.0415525839 \backslash \mathrm{C},-1.6748157122$ $, 1.3858915934,0.3132540009 \backslash \mathrm{C},-0.3900026894,1.9356641775,-0.1662451851 \backslash$ $\mathrm{C}, 0.5195941887,1.1588016814,-0.8621657864 \backslash \mathrm{Cl},-1.3719427003,-2.41564384$ $69,-1.1974806698 \backslash \mathrm{Cl},-3.4754635599,-0.6455562123,0.450595385 \backslash \mathrm{C},-0.14405$ $45991,3.3150815942,0.0972924299 \backslash \mathrm{N}, 0.0611626819,4.439455714,0.318821515$ $5 \backslash \mathrm{C}, 1.7141996574,1.7336262202,-1.4065447523 \backslash \mathrm{N}, 2.6577028562,2.229792939$ $1,-1.8710237043 \backslash 0,-2.4783271531,2.078724288,0.939851964 \backslash 0,1.119460858$, $-0.9470382422,-1.7663029232 \backslash \mathrm{H}, 1.9491910886,-1.5271303375,-0.964504619 \backslash$ $C, 2.6366539559,-1.9112961587,0.0760578424 \backslash \mathrm{C}, 3.1650743028,-0.6330945446$ $, 0.5294990726 \backslash \mathrm{C}, 2.5664375611,0.0643751638,1.5313494239 \backslash \mathrm{C}, 1.4111485226$, $-0.4748438221,2.3029038686 \backslash \mathrm{C}, 0.9903146457,-1.8461631749,1.8954212605 \backslash \mathrm{C}$ $, 1.5975836901,-2.5279587101,0.8907184478 \backslash \mathrm{H}, 3.3265154511,-2.5800007415$, $-0.4476675695 \backslash \mathrm{H}, 4.0198378849,-0.2127780539,0.0071728194 \backslash \mathrm{H}, 2.9615633219$ $, 1.0332148241,1.8279682466 \backslash \mathrm{H}, 1.640003161,-0.4560330928,3.3845020468 \backslash \mathrm{H}$, $0.1924617232,-2.3160621024,2.4654877393 \backslash \mathrm{H}, 1.2738274055,-3.5338710225,0$ $.6394957139 \backslash \mathrm{H}, 0.551703646,0.2249388278,2.2381800425 \backslash \backslash$ Version=IA64L-G03 RevD. 01 \State $=1-\mathrm{A} \backslash \mathrm{HF}=-1718.5504703 \backslash \mathrm{S} 2=0 . \backslash \mathrm{S} 2-1=0 . \backslash \mathrm{S} 2 \mathrm{~A}=0 . \backslash \mathrm{RMSD}=5.402 \mathrm{e}-09$ $\backslash \mathrm{RMSF}=7.136 \mathrm{e}-06 \backslash \mathrm{Thermal}=0 . \backslash \mathrm{Dipole}=1.7034209,-4.2563296,1.4667903 \backslash \mathrm{PG}=\mathrm{C} 0$ $1[\mathrm{X}(\mathrm{C} 14 \mathrm{H} 8 \mathrm{Cl} 2 \mathrm{~N} 2 \mathrm{O} 2)] \backslash \backslash @$ 


\section{The $A C D / D D Q$ reaction $\left(A C D=D H_{2}\right)$}

Nitrosobenzene (PhNO)

$1 \backslash 1 \backslash G I N C-A C 25 \backslash F O p t \backslash R B 3 L Y P \backslash 6-31+G(d, p) \backslash C 6 H 5 N 101 \backslash B U C 562 \backslash 04-J u l-2006 \backslash 0 \backslash \backslash \#$

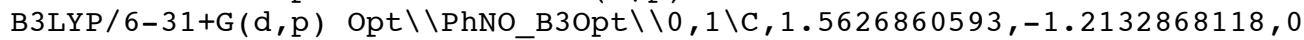
$.0000076696 \backslash \mathrm{C}, 0.1673182437,-\overline{1} .199161359,-0.0000071757 \backslash \mathrm{C},-0.5095840607$, $0.0266111908,-0.0000191342 \backslash \mathrm{C}, 0.1899232826,1.2442738265,-0.0000146428 \backslash \mathrm{C}$ $, 1.5799237991,1.2212233115,-0.0000022441 \backslash \mathrm{C}, 2.2651637563,-0.0047359533$, $0.0000102547 \backslash \mathrm{H}, 2.0998385364,-2.1566519437,0.0000162929 \backslash \mathrm{H},-0.4151314342$ $,-2.1155460141,-0.0000110877 \backslash \mathrm{H},-0.369095757,2.1739898842,-0.0000225066$ $\backslash \mathrm{H}, 2.1396960983,2.1518520317,0.0000014785 \backslash \mathrm{H}, 3.3513753982,-0.0122728278$ $, 0.0000224723 \backslash \mathrm{N},-1.9449122631,-0.0710915785,-0.0000351714 \backslash 0,-2.5426976$ $589,0.9958552437,-0.0000522055 \backslash \backslash$ Version=IA64L-G03RevD. $01 \backslash$ State $=1-A \backslash H F=$ $-361.5639042 \backslash \mathrm{RMSD}=7.240 \mathrm{e}-09 \backslash \mathrm{RMSF}=7.759 \mathrm{e}-05 \backslash \mathrm{Thermal}=0 . \backslash \mathrm{Dipole}=1.6052825$ $,-0.1796998,0.0000206 \backslash P G=C 01 \quad[X(C 6 H 5 N 101)] \backslash \backslash @$

\section{$\mathrm{DH} \bullet+\mathrm{PhNO} \rightarrow[\mathrm{DH}-\mathrm{PhNO}] \bullet \mathrm{TS}\left(\mathrm{CH}_{3} \mathrm{CN}\right)$}

$1 \backslash 1 \backslash G I N C-A C 3 \backslash F T S \backslash U B 3 L Y P \backslash 6-31+G(d, p) \backslash C 19 H 15 N 201(2) \backslash B U C 562 \backslash 01-S e p-2006 \backslash 0$ $\backslash \backslash \#$ B3LYP/6-31+G(d,p) Opt=(TS,CalcFC, NoEigenTest, GDIIS ) SCRF=( IEFPCM, R ead, Solvent $=\mathrm{CH} 3 \mathrm{CN}) \backslash \backslash \mathrm{PhNO}$ C13H9NH_rad_TS_B3Opt_CH3CN $\backslash \backslash 0,2 \backslash \mathrm{C}, 1.067160638$ $4,3.5130155003,-0.003456 \overline{1} 128 \backslash \mathrm{C},-\overline{0} .09 \overline{0} 43 \overline{4} 0851, \overline{3} .0746185389,0.6255764351$ $\backslash \mathrm{C},-0.7012267023,1.8778518635,0.2095534404 \backslash \mathrm{C},-0.1322905319,1.103578468$ $4,-0.8445921127 \backslash C, 1.0447401113,1.5893975621,-1.4680559301 \backslash C, 1.63549193$ $72,2.7730167584,-1.0594228208 \backslash \mathrm{C},-0.7597711505,-0.1202758218,-1.2163087$ $818 \backslash \mathrm{C},-2.0352548182,-0.4575187356,-0.6827354209 \backslash \mathrm{C},-2.5737042747,0.3353$ $98215,0.3687802506 \backslash \mathrm{C},-3.8159826633,0.0065980966,0.9447596978 \backslash \mathrm{H},-4.2158$ $866712,0.6223067814,1.7463283432 \backslash C,-4.5216849058,-1.0913632735,0.47663$ $45145 \backslash \mathrm{C},-4.0067936665,-1.8866003055,-0.568670289 \backslash \mathrm{C},-2.7811996571,-1.57$ $48018862,-1.1293742773 \backslash \mathrm{H},-2.2717891094,2.005044285,1.5391050575 \backslash \mathrm{H}, 1.53$ $18157041,4.4396690512,0.3203794909 \backslash \mathrm{H},-0.5361112818,3.6520625474,1.4315$ $34815 \backslash \mathrm{H}, 1.4777189423,1.0102207198,-2.2775883855 \backslash \mathrm{H}, 2.5365767722,3.12988$ $89808,-1.548260869 \backslash \mathrm{H},-5.4816179222,-1.3375070409,0.9211631693 \backslash \mathrm{H},-4.569$ $2438959,-2.7442766377,-0.9244584908 \backslash \mathrm{H},-2.3647457661,-2.1879848849,-1.9$ $235472339 \backslash \mathrm{N},-1.8729916888,1.4435772346,0.7915855507 \backslash \mathrm{H},-0.3916725659,-0$ $.6605102481,-2.0795810585 \backslash \mathrm{N}, 0.4256835209,-1.7814874719,0.1189583771 \backslash \mathrm{C}$, $1.796627618,-1.439655527,0.2490070364 \backslash \mathrm{C}, 2.3823676227,-1.0369596419,1.4$ $648883371 \backslash \mathrm{C}, 2.5868191788,-1.5164759516,-0.9124670345 \backslash \mathrm{C}, 3.739018218,-0$. $7219890288,1.5079979882 \backslash \mathrm{H}, 1.758983068,-0.9789508227,2.350608928 \backslash \mathrm{C}, 3.94$ $52622496,-1.208990321,-0.8576168631 \backslash \mathrm{H}, 2.1232114173,-1.8317936662,-1.84$ $35606523 \backslash \mathrm{C}, 4.5272042505,-0.8077605244,0.3518597774 \backslash \mathrm{H}, 4.1889593321,-0.4$ $062185042,2.4458774191 \backslash \mathrm{H}, 4.5521407667,-1.2800230595,-1.7561945217 \backslash \mathrm{H}, 5$. $5847801144,-0.5629727898,0.3935616304 \backslash 0,-0.2142531059,-1.9145334601,1$. $2051265963 \backslash \backslash$ Version=IA64L-G03RevD.01 \State $=2-\mathrm{A} \backslash \mathrm{HF}=-917.7679607 \backslash \mathrm{S} 2=0.78$ $4467 \backslash \mathrm{S} 2-1=0 . \backslash \mathrm{S} 2 \mathrm{~A}=0.750443 \backslash \mathrm{RMSD}=8.660 \mathrm{e}-09 \backslash \mathrm{RMSF}=5.719 \mathrm{e}-06 \backslash \mathrm{Thermal}=0 . \backslash \mathrm{Dip}$ ole $=-0.6885039,2.4341863,-0.3812852 \backslash \mathrm{PG}=\mathrm{C} 01 \quad[\mathrm{X}(\mathrm{C} 19 \mathrm{H} 15 \mathrm{~N} 2 \mathrm{O} 1)] \backslash \backslash @$ 


\section{$\mathrm{DH}^{+}+\mathrm{PhNO} \rightarrow[\mathrm{DH}-\mathrm{PhNO}]^{+} \mathrm{TS}^{\left(\mathrm{CH}_{3} \mathrm{CN}\right)}$}

$1 \backslash 1 \backslash G I N C-A C 3 \backslash F T S \backslash R B 3 L Y P \backslash 6-31+G(d, p) \backslash C 19 H 15 N 201(1+) \backslash B U C 562 \backslash 25-A u g-2006 \backslash$ $0 \backslash \backslash \#$ B3LYP /6-31+G(d,p) Opt=(TS,CalcFC,NoEigenTest) \\PhNO_C13H9NH_catio n_TS_B3Opt $\backslash \backslash 1,1 \backslash C, 0.1247316663,3.8800626574,0.059166696 \backslash \bar{C},-0.898880653$ $8,3 . \overline{1} 637612359,0.6558254224 \backslash \mathrm{C},-1.0714818679,1.7991069673,0.3470167228 \backslash$ $\mathrm{C},-0.1730694579,1.1569748092,-0.5426241565 \backslash \mathrm{C}, 0.8470552121,1.9169987708$ $,-1.1488674636 \backslash \mathrm{C}, 0.9957062494,3.2640374562,-0.8622512906 \backslash \mathrm{C},-0.32132410$ $79,-0.3010556307,-0.7538620898 \backslash C,-1.6688685696,-0.8416936748,-0.507860$ $9502 \backslash C,-2.5282454603,-0.1449083868,0.3677075293 \backslash C,-3.7836239862,-0.684$ $6436986,0.6986776101 \backslash \mathrm{H},-4.4420129204,-0.1489235481,1.3767677333 \backslash \mathrm{C},-4.1$ $820079483,-1.8907840945,0.1384858591 \backslash \mathrm{C},-3.3472739372,-2.5782605247,-0$. $7590437801 \backslash \mathrm{C},-2.0990670923,-2.0546412753,-1.0711864018 \backslash \mathrm{H},-2.7737241561$ $, 1.57528468,1.4752980325 \backslash \mathrm{H}, 0.2452525387,4.9329033805,0.2936000853 \backslash \mathrm{H},-1$ $.5829434963,3.6501398354,1.3455999592 \backslash \mathrm{H}, 1.5184775896,1.4385184915,-1.8$ $55677883 \backslash \mathrm{H}, 1.7758099547,3.8432343521,-1.3440193836 \backslash \mathrm{H},-5.1553536307,-2$. $2988666647,0.3913083653 \backslash \mathrm{H},-3.6728367142,-3.5149114284,-1.1981511586 \backslash \mathrm{H}$, $-1.4410633507,-2.5867012821,-1.7533538809 \backslash \mathrm{N},-2.1308226886,1.0859378896$ $, 0.8660698447 \backslash \mathrm{H}, 0.1488813642,-0.6774305802,-1.6585469271 \backslash \mathrm{N}, 0.673249697$ $8,-0.948616074,0.488666597 \backslash \mathrm{C}, 2.0862893215,-0.9880606022,0.4195525554 \backslash \mathrm{C}$ , $2.8309501753,-0.986432706,1.6182559064 \backslash \mathrm{C}, 2.7204059284,-1.1074115535,-$ $0.8343455423 \backslash \mathrm{C}, 4.211812808,-1.0938088901,1.5516203123 \backslash \mathrm{H}, 2.3148396606$,$0.8938729816,2.5666616268 \backslash \mathrm{C}, 4.1043022589,-1.2279391688,-0.8795681043 \backslash \mathrm{H}$ , $2.1491060199,-1.1378702633,-1.7534713144 \backslash \mathrm{C}, 4.8490338069,-1.2157696039$ $, 0.306747222 \backslash \mathrm{H}, 4.798552049,-1.0828642516,2.464017119 \backslash \mathrm{H}, 4.604394964,-1$. $3364883112,-1.8359235773 \backslash \mathrm{H}, 5.9301880752,-1.3028073859,0.2626398034 \backslash 0,0$ $.110847698,-1.1213749446,1.5536819018 \backslash \backslash$ Version=IA64L-G03RevD.01 $\backslash$ State= $1-\mathrm{A} \backslash \mathrm{HF}=-917.5424741 \backslash \mathrm{RMSD}=8.792 \mathrm{e}-09 \backslash \mathrm{RMSF}=3.869 \mathrm{e}-06 \backslash \mathrm{Thermal}=0 . \backslash \mathrm{Dipole}=1$. $1550743,0.0082021,-0.0359982 \backslash P G=C 01 \quad[X(C 19 H 15 \mathrm{~N} 201)] \backslash \backslash @$

\section{[DH-PhNO]•}

$1 \backslash 1 \backslash G I N C-A C 15 \backslash F O p t \backslash U B 3 L Y P \backslash 6-31+G(d, p) \backslash C 19 H 15 N 2 O 1(2) \backslash B U C 562 \backslash 05-J u 1-2006$

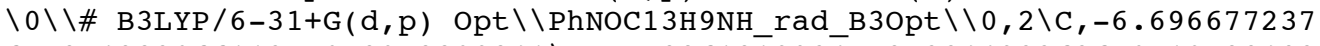
$6,-3.4382066112,-0.3058809841 \backslash \mathrm{C},-5.38613 \overline{1} 992 \overline{4},-3.2244922626,0.10583432$ $\backslash C,-4.8515964854,-1.9232722189,0.1245089323 \backslash C,-5.6539207727,-0.8328690$ $649,-0.2610080707 \backslash \mathrm{C},-6.9655705147,-1.0743407418,-0.6865041283 \backslash \mathrm{C},-7.495$ $7584889,-2.3627119846,-0.7152086865 \backslash C,-5.120385401,0.5797379503,-0.150$ $0022079 \backslash \mathrm{C},-3.6080073139,0.6307845512,-0.0996318328 \backslash \mathrm{C},-2.8750742384,-0$. $5092605055,0.2804227297 \backslash \mathrm{C},-1.4769562661,-0.4278685375,0.4142092304 \backslash \mathrm{H},-$ $0.9162620409,-1.3100667431,0.7140102401 \backslash C,-0.8166110034,0.7683690081,0$ $.1579529551 \backslash \mathrm{C},-1.5340452377,1.9022720194,-0.2449416472 \backslash \mathrm{C},-2.9197117804$ $, 1.8200527362,-0.36736137 \backslash \mathrm{H},-2.9691757453,-2.5222708325,0.7116706208 \backslash \mathrm{H}$ $,-7.0949983351,-4.4486095439,-0.3154397043 \backslash \mathrm{H},-4.763803154,-4.062601018$ $6,0.4104854277 \backslash \mathrm{H},-7.5845103322,-0.2310802475,-0.9834870969 \backslash \mathrm{H},-8.516514$ $4122,-2.5282687931,-1.0441649658 \backslash \mathrm{H}, 0.2632006513,0.8154121672,0.2649972$ $661 \backslash \mathrm{H},-1.019708722,2.8349099218,-0.4528072465 \backslash \mathrm{H},-3.4871197636,2.700177$ $6412,-0.660274779 \backslash \mathrm{N},-3.5308501728,-1.7131085461,0.4935132274 \backslash \mathrm{H},-5.4746$ $139853,1.1679925054,-0.9953920472 \backslash \mathrm{N},-5.684247843,1.231191482,1.0890498$ $816 \backslash \mathrm{C},-6.5059261197,2.380294175,1.0868758921 \backslash \mathrm{C},-6.9560158304,2.8698395$ $678,2.3308822128 \backslash \mathrm{C},-6.8899233028,3.0499439325,-0.0933840728 \backslash \mathrm{C},-7.76725$ $04672,3.9982003443,2.3838885664 \backslash \mathrm{H},-6.6546848003,2.3474897127,3.2297156$ $422 \backslash \mathrm{C},-7.7043576385,4.1804398714,-0.0195395174 \backslash \mathrm{H},-6.5679710456,2.70972$ $34295,-1.0695845524 \backslash \mathrm{C},-8.1494695762,4.6642497555,1.2130156716 \backslash \mathrm{H},-8.104$ $9024485,4.3616895495,3.3502591884 \backslash \mathrm{H},-7.9902793237,4.6836391217,-0.9386$ $316002 \backslash \mathrm{H},-8.7828706611,5.5444661846,1.2613496867 \backslash \mathrm{O},-5.3750444782,0.675$ $5756744,2.1997406688 \backslash \backslash$ Version=IA64L-G03RevD.01 \State $=2-A \backslash H F=-917.78346$ $96 \backslash \mathrm{S} 2=0.763349 \backslash \mathrm{S} 2-1=0 . \backslash \mathrm{S} 2 \mathrm{~A}=0.750135 \backslash \mathrm{RMSD}=3.641 \mathrm{e}-09 \backslash \mathrm{RMSF}=2.563 \mathrm{e}-06 \backslash \mathrm{Ther}$ $\mathrm{mal}=0 . \backslash \mathrm{Dipole}=0.1483059,-0.1234395,-0.7568115 \backslash \mathrm{PG}=\mathrm{C} 01 \quad[\mathrm{X}(\mathrm{C} 19 \mathrm{H} 15 \mathrm{~N} 201)] \backslash \backslash$ a 


\section{$[\mathrm{DH}-\mathrm{PhNO}]^{+}$}

$1 \backslash 1 \backslash G I N C-A C 10 \backslash F O p t \backslash R B 3 L Y P \backslash 6-31+G(d, p) \backslash C 19 H 15 N 2 O 1(1+) \backslash B U C 562 \backslash 05-J u 1-200$

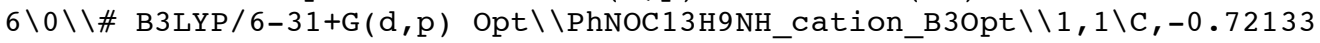
$86977,3.8322277174,-0.130272609 \backslash \mathrm{C},-1.5651 \overline{1} 81337, \overline{2} .940072413,0.50980073$ $38 \backslash \mathrm{C},-1.4558890138,1.559680003,0.2449270419 \backslash \mathrm{C},-0.4561763845,1.08932576$ $25,-0.6424655001 \backslash \mathrm{C}, 0.374670252,2.0193069393,-1.2930362685 \backslash \mathrm{C}, 0.24331290$ $5,3.3784153987,-1.051593603 \backslash C,-0.2931731301,-0.3894658015,-0.790758159$ $1 \backslash \mathrm{C},-1.5353495546,-1.1680029574,-0.5501425193 \backslash \mathrm{C},-2.50745218,-0.6303168$ $043,0.3164314215 \backslash \mathrm{C},-3.6468339807,-1.3825399316,0.6479815215 \backslash \mathrm{H},-4.39268$ $20484,-0.969021433,1.3210564393 \backslash C,-3.8238004636,-2.6437622498,0.091477$ $0986 \backslash \mathrm{C},-2.8797702799,-3.172166263,-0.8020300083 \backslash \mathrm{C},-1.7410953696,-2.434$ $2774937,-1.1134892998 \backslash \mathrm{H},-3.0582236827,1.0260251494,1.4131905941 \backslash \mathrm{H},-0.8$ $176995649,4.8945199221,0.0700501509 \backslash \mathrm{H},-2.3240924985,3.2970970584,1.200$ $2967466 \backslash \mathrm{H}, 1.1212812493,1.6672421176,-1.9985823922 \backslash \mathrm{H}, 0.8778494429,4.089$ $2588992,-1.5694729193 \backslash \mathrm{H},-4.7094648305,-3.2175318876,0.3446722415 \backslash \mathrm{H},-3$. $030716594,-4.1532193016,-1.2389437482 \backslash \mathrm{H},-0.9970147404,-2.8444741761,-1$ $.7913560897 \backslash \mathrm{N},-2.3375618227,0.6587761121,0.8049764525 \backslash \mathrm{H}, 0.219925749,-0$ $.6804877515,-1.7044423873 \backslash \mathrm{N}, 0.7441632121,-0.7465434609,0.4080950851 \backslash \mathrm{C}$, $2.1420172714,-0.5390153621,0.3129914084 \backslash \mathrm{C}, 2.8806883805,-0.3879982969,1$ $.5058900388 \backslash \mathrm{C}, 2.7838394574,-0.5656702594,-0.9430738443 \backslash \mathrm{C}, 4.2589813403$, $-0.2546931276,1.4325584806 \backslash \mathrm{H}, 2.3607456961,-0.3708858716,2.4562996753 \backslash \mathrm{C}$ $, 4.1672854753,-0.4431074603,-0.9939508216 \backslash \mathrm{H}, 2.2282312856,-0.7082888985$ $,-1.860879902 \backslash \mathrm{C}, 4.9040102271,-0.2830063392,0.1865258137 \backslash \mathrm{H}, 4.8368300538$ $,-0.1272544351,2.3417369843 \backslash \mathrm{H}, 4.6741457763,-0.4774707939,-1.9522897151$ $\backslash \mathrm{H}, 5.9835235081,-0.1808990554,0.1365551833 \backslash 0,0.234675688,-0.9980890806$ , $1.4893586753 \backslash \backslash$ Version=IA64L-G03RevD. 01 \State $=1-\mathrm{A} \backslash \mathrm{HF}=-917.5426014 \backslash \mathrm{RMSD}$ $=6.575 \mathrm{e}-09 \backslash \mathrm{RMSF}=1.945 \mathrm{e}-05 \backslash \mathrm{Thermal}=0 . \backslash \mathrm{Dipole}=1.1407759,0.2223415,-0.079$ $6523 \backslash \mathrm{PG}=\mathrm{C} 01 \quad[\mathrm{X}(\mathrm{C} 19 \mathrm{H} 15 \mathrm{~N} 2 \mathrm{O} 1)] \backslash \backslash @$

\section{The $C H D / P B Q$ reaction}

\section{CHD/PBQ TS1 (UB3-LYP, gas phase)}

$1 \backslash 1 \backslash \mathrm{GINC}-\mathrm{AC} 14 \backslash \mathrm{FTS} \backslash \mathrm{UB} 3 \mathrm{LYP} \backslash 6-31+\mathrm{G}(\mathrm{d}, \mathrm{p}) \backslash \mathrm{C} 12 \mathrm{H} 12 \mathrm{O} 2 \backslash \mathrm{BUC} 562 \backslash 20-\mathrm{Sep}-2005 \backslash 0 \backslash \backslash \#$ UB3LYP $/ 6-31+G(D, P)$ GUESS=(MIX, ALWAYS ) OPT=(TS, CALCFC , NOEIGENTEST $) \backslash \backslash O C 6$

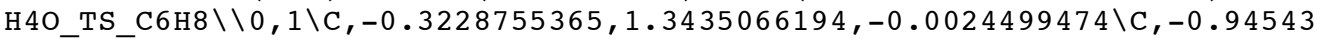
$025 \overline{1} 5, \overline{0} .9566564625,1.23889177 \backslash \mathrm{C},-2.0964592531,0.226429184,1.2464719782$ $\backslash C,-2.7452303927,-0.1991730008,-0.0003073556 \backslash C,-2.0962047017,0.2225270$ $879,-1.2482724789 \backslash \mathrm{C},-0.9451361888,0.9526908671,-1.2427219912 \backslash \mathrm{H},-0.4707$ $568288,1.2847908391,2.1587096696 \backslash \mathrm{H},-2.5872304212,-0.0589705178,2.17214$ $99809 \backslash \mathrm{H},-2.5867608742,-0.0657867633,-2.1731605481 \backslash \mathrm{H},-0.4702058408,1.27$ $78708589,-2.1634575254 \backslash 0,0.7676554282,2.0634916545,-0.0034786022 \backslash 0,-3$. $7934231856,-0.8682844816,0.0006384862 \backslash \mathrm{H}, 1.7386794345,1.4384943306,-0.0$ $018342158 \backslash \mathrm{C}, 2.7650110207,0.4308075087,-0.0003160643 \backslash \mathrm{C}, 2.4134665774,-0$. $2380032323,1.2372358018 \backslash \mathrm{C}, 1.5725155015,-1.302906204,1.2542455054 \backslash \mathrm{C}, 1.0$ $266569116,-1.9236523703,0.003875092 \backslash C, 1.5723921091,-1.3072559812,-1.24$ $86984381 \backslash \mathrm{C}, 2.4133570947,-0.2423206149,-1.2354836312 \backslash \mathrm{H}, 3.6364044021,1.0$ $851348759,-0.0015166662 \backslash \mathrm{H}, 2.7864969726,0.1713307828,2.1725667566 \backslash \mathrm{H}, 1.2$ $857562369,-1.7514976928,2.2020138755 \backslash \mathrm{H}, 1.2107144205,-3.0112558333,0.00$ $57479457 \backslash \mathrm{H}, 1.2855224427,-1.7591385842,-2.1948679193 \backslash \mathrm{H}, 2.7862910012,0.1$ $637578084,-2.172270264 \backslash \mathrm{H},-0.081146231,-1.8522254438,0.0038183947 \backslash \backslash$ Vers ion $=\mathrm{IA} 64 \mathrm{~L}-\mathrm{G} 03 \mathrm{RevC} .02 \backslash \mathrm{State}=1-\mathrm{A} \backslash \mathrm{HF}=-614.8813435 \backslash \mathrm{S} 2=0.204111 \backslash \mathrm{S} 2-1=0 . \backslash \mathrm{S} 2 \mathrm{~A}$ $=0.009335 \backslash \mathrm{RMSD}=3.729 \mathrm{e}-09 \backslash \mathrm{RMSF}=1.142 \mathrm{e}-05 \backslash \mathrm{Dipole}=1.9998619,-0.1844206,0$. $0008659 \backslash \mathrm{PG}=\mathrm{C} 01 \quad[\mathrm{X}(\mathrm{C} 12 \mathrm{H} 12 \mathrm{O} 2)] \backslash \backslash @$ 


\section{CHD/PBQ TS1 (UB3-LYP, heptane)}

$1 \backslash 1 \backslash G I N C-A C 24 \backslash F T S \backslash U B 3 L Y P \backslash 6-31+G(d, p) \backslash C 12 H 1202 \backslash B U C 562 \backslash 31-J a n-2006 \backslash 0 \backslash \backslash \#$ UB3LYP $/ 6-31+G(D, P)$ GUESS=(MIX, ALWAYS $)$ OPT=(TS, CALCFC, NOEIGENTEST $)$ SCRF $=($ READ , IEFPCM, SOLVENT $=$ HEPTANE $) \backslash \backslash O C 6 \mathrm{H} 40$ TS C6 H8 B3Opt Heptane $02 \backslash \backslash 0,1 \backslash \mathrm{C}$ $,-0.372275901,-1.3265245496,-0.0103545744 \backslash C,-0.9744082002,-0.897513088$ $6,-1.2482263271 \backslash \mathrm{C},-2.0915907481,-0.1159899979,-1.2488597392 \backslash \mathrm{C},-2.72310$ $717,0.3231987749,0.0004160189 \backslash \mathrm{C},-2.0944450673,-0.1362303984,1.24382085$ $67 \backslash \mathrm{C},-0.9771143125,-0.9174303147,1.2329675658 \backslash \mathrm{H},-0.5149500753,-1.23618$ $75357,-2.1720496281 \backslash \mathrm{H},-2.5657189941,0.1999504178,-2.1735338064 \backslash \mathrm{H},-2.57$ $06072569,0.1647112357,2.1724407305 \backslash \mathrm{H},-0.5195720923,-1.2709105333,2.152$ $1828435 \backslash 0,0.6817074612,-2.0979871155,-0.0153430077 \backslash 0,-3.7468138576,1.0$ $341364713,0.00500583 \backslash \mathrm{H}, 1.68670374,-1.5122900716,-0.0095962766 \backslash \mathrm{C}, 2.7398$ $870953,-0.5500364416,-0.0009698285 \backslash \mathrm{C}, 2.414317015,0.1410688995,-1.23349$ $91089 \backslash \mathrm{C}, 1.6131442781,1.2368274679,-1.2411991613 \backslash \mathrm{C}, 1.0951413684,1.86922$ $71244,0.0137758373 \backslash C, 1.6087889548,1.2186109225,1.2611983979 \backslash C, 2.410128$ $609,0.1231243009,1.2403259046 \backslash \mathrm{H}, 3.5870388579,-1.2362066941,-0.00452111$ $01 \backslash \mathrm{H}, 2.7715787331,-0.275011497,-2.1720171305 \backslash \mathrm{H}, 1.3436810747,1.70395952$ $91,-2.1851563183 \backslash \mathrm{H}, 1.3269093036,2.9479210245,0.0220305243 \backslash \mathrm{H}, 1.33597750$ $46,1.671897579,2.2109235957 \backslash \mathrm{H}, 2.7641807422,-0.3065355425,2.1739281029 \backslash$ $\mathrm{H},-0.0151658941,1.8495110456,0.011690845 \backslash \backslash$ Version=IA64L-G03RevC.02 $\backslash$ Sta $\mathrm{te}=1-\mathrm{A} \backslash \mathrm{HF}=-614.8853169 \backslash \mathrm{S} 2=0.152552 \backslash \mathrm{S} 2-1=0 . \backslash \mathrm{S} 2 \mathrm{~A}=0.004831 \backslash \mathrm{RMSD}=8.122 \mathrm{e}-09$ $\backslash \mathrm{RMSF}=3.137 \mathrm{e}-06 \backslash \mathrm{Dipole}=2.3906629,0.1150525,0.0027814 \backslash \mathrm{PG}=\mathrm{C} 01][\mathrm{X}(\mathrm{C} 12 \mathrm{H} 120$ $2)] \backslash \backslash @$

\section{CHD/PBQ TS1 (UB3-LYP, ether)}

$1 \backslash 1 \backslash \mathrm{GINC}-\mathrm{AC} 14 \backslash \mathrm{FTS} \backslash \mathrm{UB} 3 \mathrm{LYP} \backslash 6-31+\mathrm{G}(\mathrm{d}, \mathrm{p}) \backslash \mathrm{C} 12 \mathrm{H} 12 \mathrm{O} 2 \backslash \mathrm{BUC5} 62 \backslash 31-\mathrm{Jan}-2006 \backslash 0 \backslash \backslash \#$ $\mathrm{UB} 3 \mathrm{LYP} / 6-31+\mathrm{G}(\mathrm{D}, \mathrm{P})$ GUESS=(MIX, ALWAYS $)$ OPT $=(\mathrm{TS}$, CALCFC, NOEIGENTEST $)$ SCRF

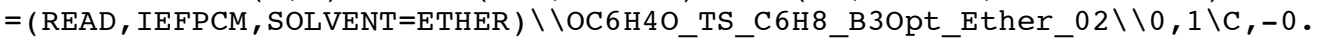
$3399931037,-1.3300829735,-0.019541 \backslash C_{,}^{-}-0.9425 \overline{8} 90695,-0.89 \overline{1} 4799265,-1.25$ $4298 \backslash C,-2.0647490091,-0.116221839,-1.248241 \backslash C,-2.7009829762,0.30479521$ $07,0.002529 \backslash \mathrm{C},-2.0723010125,-0.1602788384,1.2414 \backslash \mathrm{C},-0.9491100728,-0.93$ $3731926,1.226493 \backslash \mathrm{H},-0.481602095,-1.2175999624,-2.182381 \backslash \mathrm{H},-2.536885984$ $, 0.2049201979,-2.172832 \backslash \mathrm{H},-2.54963799,0.1283821988,2.173984 \backslash \mathrm{H},-0.49236$ $01007,-1.2911939616,2.145093 \backslash 0,0.7164538365,-2.0967800559,-0.029539 \backslash 0$, $-3.7357139215,1.0068922914,0.01183 \backslash \mathrm{H}, 1.7249638834,-1.4947921345,-0.017$ $756 \backslash \mathrm{C}, 2.7409849599,-0.5137162138,-0.000146 \backslash \mathrm{C}, 2.3979780134,0.172301813$, $-1.23062 \backslash \mathrm{C}, 1.5653970971,1.2454678779,-1.233878 \backslash \mathrm{C}, 1.0357891452,1.861661$ $9192,0.021993 \backslash \mathrm{C}, 1.5526870946,1.2130788789,1.266756 \backslash \mathrm{C}, 2.385865011,0.140$ $4538139,1.244042 \backslash \mathrm{H}, 3.6070369081,-1.1781542813,-0.004393 \backslash \mathrm{H}, 2.7659779819$ $,-0.2315952157,-2.170601 \backslash \mathrm{H}, 1.2833721333,1.7086368999,-2.176493 \backslash \mathrm{H}, 1.249$ $2602297,2.9454189026,0.037078 \backslash \mathrm{H}, 1.2607271288,1.6514039017,2.218178 \backslash \mathrm{H}, 2$ $.7445539776,-0.287548214,2.176928 \backslash \mathrm{H},-0.0751818575,1.8277440059,0.01594$ $4 \backslash \backslash$ Version=IA64L-G03RevC.02 $\backslash$ State $=1-A \backslash H F=-614.8914392 \backslash \mathrm{S} 2=0.052331 \backslash \mathrm{S} 2-1$ $=0 . \backslash \mathrm{S} 2 \mathrm{~A}=0.000501 \backslash \mathrm{RMSD}=6.909 \mathrm{e}-09 \backslash \mathrm{RMSF}=6.586 \mathrm{e}-06 \backslash \mathrm{Dipole}=2.913656,0.15968$ $64,0.0056142 \backslash$ Polar $=407.1053726,-1.1678724,179.8763216,0.0928865,-0.024$ $7502,167.2006358 \backslash \mathrm{PG}=\mathrm{C} 01 \quad[\mathrm{X}(\mathrm{C} 12 \mathrm{H} 12 \mathrm{O} 2)] \backslash \backslash @$ 


\section{CHD/PBQ TS1 (UB3-LYP, acetone)}

$1 \backslash 1 \backslash G I N C-A C 15 \backslash F T S \backslash U B 3 L Y P \backslash 6-31+G(d, p) \backslash C 12 H 1202 \backslash B U C 562 \backslash 31-J a n-2006 \backslash 0 \backslash \backslash \#$ UB3LYP $/ 6-31+G(D, P)$ GUESS=(MIX, ALWAYS $)$ OPT=(TS, CALCFC, NOEIGENTEST $)$ SCRF $=($ READ , IEFPCM, SOLVENT $=$ ACETONE $) \backslash \backslash O C 6 \mathrm{H} 40$ TS_C6H8_B3Opt_Acetone_02 $\backslash \backslash 0,1 \backslash \mathrm{C}$ $,-0.3225486149,-1.3289841979,-0.006202 \overline{2} 08 \backslash \bar{C},-0.92841 \overline{1} 3583,-0.904403469$ $9,-1.2449056159 \backslash \mathrm{C},-2.0543941578,-0.1344977956,-1.2452548783 \backslash \mathrm{C},-2.69261$ $84617,0.2949792878,0.0008317557 \backslash \mathrm{C},-2.0569899385,-0.1489487604,1.243194$ $9762 \backslash \mathrm{C},-0.9306255319,-0.9182071585,1.2361289804 \backslash \mathrm{H},-0.4671158341,-1.236$ $8193637,-2.1703265126 \backslash \mathrm{H},-2.5270115339,0.1745539173,-2.1734771341 \backslash \mathrm{H},-2$. $5314472417,0.1494266866,2.1739681089 \backslash \mathrm{H},-0.4709120218,-1.2609816077,2.1$ $58553647 \backslash 0,0.7333492605,-2.0945896205,-0.0093434534 \backslash 0,-3.7370102627,0$. $9850238061,0.0037374041 \backslash \mathrm{H}, 1.7448345999,-1.4870355186,-0.0061199319 \backslash \mathrm{C}, 2$ $.7422872488,-0.4962582847,-0.0008134616 \backslash \mathrm{C}, 2.3842961284,0.1733779615,-1$ $.235837812 \backslash C, 1.5351377792,1.2337652801,-1.24450519 \backslash C, 1.0054762315,1.85$ $53625689,0.0077255566 \backslash \mathrm{C}, 1.5327236878,1.2229657643,1.2555560953 \backslash \mathrm{C}, 2.381$ $9658691,0.1626507473,1.2392716225 \backslash \mathrm{H}, 3.6180506481,-1.1470574447,-0.0027$ $848969 \backslash \mathrm{H}, 2.7534125002,-0.2337964316,-2.1736891369 \backslash \mathrm{H}, 1.242303441,1.6839$ $305471,-2.1898838208 \backslash \mathrm{H}, 1.2076940846,2.940700218,0.0125260947 \backslash \mathrm{H}, 1.23802$ $07755,1.6648591189,2.2042491752 \backslash \mathrm{H}, 2.749309074,-0.2525983969,2.17427078$ $46 \backslash \mathrm{H},-0.1056437632,1.8105331326,0.0064230912 \backslash \backslash$ Version=IA64L-G03RevC.02 $\backslash \mathrm{State}=1-\mathrm{A} \backslash \mathrm{HF}=-614.8922094 \backslash \mathrm{S} 2=0 . \backslash \mathrm{S} 2-1=0 . \backslash \mathrm{S} 2 \mathrm{~A}=0 . \backslash \mathrm{RMSD}=3.623 \mathrm{e}-09 \backslash \mathrm{RMSF}=4$. $803 e-06 \backslash \mathrm{Dipole}=3.1566931,0.1814146,0.0006876 \backslash \mathrm{PG}=\mathrm{C} 01 \quad[\mathrm{X}(\mathrm{C} 12 \mathrm{H} 12 \mathrm{O} 2)] \backslash \backslash @$

\section{CHD/PBQ TS1 (UB3-LYP, $\mathrm{CH}_{3} \mathrm{CN}$ )}

$1 \backslash 1 \backslash G I N C-A C 12 \backslash F T S \backslash U B 3 L Y P \backslash 6-31+G(d, p) \backslash C 12 H 1202 \backslash B U C 562 \backslash 01-A u g-2006 \backslash 0 \backslash \backslash \#$ $\mathrm{UB} 3 \mathrm{LYP} / 6-31+\mathrm{G}(\mathrm{d}, \mathrm{p})$ Guess=(Mix,Always) Opt=(TS, CalcFC,NoEigenTest) SCRF $=($ Read, IEFPCM, Solvent $=\mathrm{CH} 3 \mathrm{CN}) \backslash \backslash \mathrm{OC} 6 \mathrm{H} 4 \mathrm{O} \_\mathrm{TS} \_\mathrm{C} 6 \mathrm{H} 8$ B3Opt_CH3CN_02 $\backslash \backslash 0,1 \backslash \mathrm{C},-0$. $3219845483,-1.3298085393,-0.00649896 \overline{3} 5 \backslash \bar{C},-0 . \overline{9} 27860 \overline{3} 871,-\overline{0} .9050078932,-$ $1.2452065656 \backslash \mathrm{C},-2.0540100359,-0.1352587467,-1.2454499432 \backslash \mathrm{C},-2.69172232$ $03,0.2944318607,0.0005674865 \backslash C,-2.0559314059,-0.1488853878,1.242808581$ $8 \backslash \mathrm{C},-0.9296522548,-0.9183554136,1.2358562041 \backslash \mathrm{H},-0.4665572422,-1.237126$ $114,-2.1707514848 \backslash \mathrm{H},-2.5263738002,0.1738540725,-2.1738106054 \backslash \mathrm{H},-2.5296$ $912048,0.1501094306,2.1737654775 \backslash \mathrm{H},-0.4696339824,-1.2603983737,2.15842$ $36149 \backslash 0,0.7335502567,-2.0955467784,-0.0097669883 \backslash 0,-3.7363878452,0.984$ $8171323,0.0035614337 \backslash \mathrm{H}, 1.7468428549,-1.4863589858,-0.0061107586 \backslash \mathrm{C}, 2.74$ $09216344,-0.4964243477,-0.0006811423 \backslash \mathrm{C}, 2.3828890278,0.173447997,-1.235$ $7765281 \backslash \mathrm{C}, 1.5339339759,1.2340119903,-1.2442240602 \backslash \mathrm{C}, 1.0050388799,1.855$ $8617136,0.0081080988 \backslash \mathrm{C}, 1.5319133482,1.2232528227,1.2558854462 \backslash \mathrm{C}, 2.3807$ $807327,0.1626804391,1.2395912058 \backslash \mathrm{H}, 3.6176505978,-1.1460984504,-0.00281$ $22554 \backslash \mathrm{H}, 2.751673019,-0.2338775476,-2.1736953532 \backslash \mathrm{H}, 1.2409822576,1.68426$ $67653,-2.1895380133 \backslash \mathrm{H}, 1.2079409893,2.941119872,0.0129279894 \backslash \mathrm{H}, 1.237487$ $3139,1.6653088741,2.2046035399 \backslash \mathrm{H}, 2.7480090181,-0.2527511848,2.17456190$ $24 \backslash \mathrm{H},-0.1060828793,1.8118907929,0.0069556807 \backslash \backslash$ Version=IA64L-G0 3RevD. 01 $\backslash$ State $=1-\mathrm{A} \backslash \mathrm{HF}=-614.8926532 \backslash \mathrm{S} 2=0 . \backslash \mathrm{S} 2-1=0 . \backslash \mathrm{S} 2 \mathrm{~A}=0 . \backslash \mathrm{RMSD}=1.591 \mathrm{e}-09 \backslash \mathrm{RMSF}=3$. $002 \mathrm{e}-06 \backslash \mathrm{Thermal}=0 . \backslash \mathrm{Dipole}=3.1979866,0.1855481,0.0012506 \backslash \mathrm{PG}=\mathrm{C} 01 \quad[\mathrm{X}(\mathrm{C} 12 \mathrm{H}$ $1202)] \backslash \backslash$ @ 


\section{The $D H P / P B Q$ reaction}

DHP/PBQ TS1 (UB3-LYP, gas phase)

$1 \backslash 1 \backslash G I N C-A C 9 \backslash F T S \backslash U B 3 L Y P \backslash 6-31+G(d, p) \backslash C 11 \mathrm{H} 11 \mathrm{~N} 102 \backslash B U C 562 \backslash 20-$ Sep-2005 $\backslash 0 \backslash \backslash \#$ UB3LYP $/ 6-31+G(D, P)$ GUESS $=($ MIX, ALWAYS $)$ OPT $=($ TS , CALCFC, NOEIGENTEST $) \backslash \backslash O C$

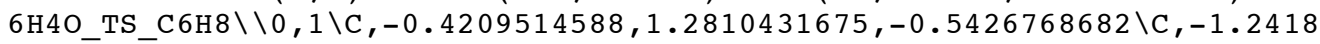
$254497, \overline{1} .5732021169,0.6054847894 \backslash \mathrm{C},-2.3798933947,0.8634478508,0.861301$ $6691 \backslash \mathrm{C},-2.7915004198,-0.2546773893,0.0100994077 \backslash \mathrm{C},-1.9526626534,-0.514$ $6237114,-1.1660251008 \backslash \mathrm{C},-0.8326175992,0.2219329618,-1.4242053621 \backslash \mathrm{H},-0$. $9305745052,2.3971357064,1.2426158049 \backslash \mathrm{H},-3.0151758834,1.089436371,1.712$ $6640078 \backslash \mathrm{H},-2.2811555974,-1.3086685906,-1.8307699438 \backslash \mathrm{H},-0.2216005564,0$. $0392440321,-2.3042820542 \backslash 0,0.6578283064,1.9762832653,-0.794409693 \backslash 0,-3$ $.796388714,-0.9489050541,0.2651540537 \backslash \mathrm{H}, 1.6573239513,1.4456425151,-0.3$ $859508773 \backslash \mathrm{C}, 2.5931620977,0.6517463549,0.2267510948 \backslash \mathrm{C}, 1.8485129317,0.22$ $01165032,1.4028164989 \backslash \mathrm{C}, 1.2529895084,-1.0036014307,1.4488562992 \backslash \mathrm{N}, 1.44$ $19063723,-1.9187971525,0.4387659224 \backslash C, 2.2620525574,-1.6243502819,-0.63$ $55862489 \backslash \mathrm{C}, 2.8673260764,-0.4110457096,-0.7346173571 \backslash \mathrm{H}, 3.37087911,1.403$ $7965735,0.374273755 \backslash \mathrm{H}, 1.7014854131,0.8997366893,2.2346130585 \backslash \mathrm{H}, 0.62588$ $85026,-1.3261697749,2.2709518406 \backslash \mathrm{H}, 0.9567562937,-2.8026458041,0.471228$ $6345 \backslash \mathrm{H}, 2.4004967762,-2.4195674815,-1.3578297206 \backslash \mathrm{H}, 3.5232619736,-0.2245$ $284518,-1.5780237791 \backslash \backslash$ Version=IA64L-G03RevC.02 \State $=1-A \backslash H F=-630.93730$ $6 \backslash S 2=0.19451 \backslash \mathrm{S} 2-1=0 . \backslash \mathrm{S} 2 \mathrm{~A}=0.004972 \backslash \mathrm{RMSD}=4.610 \mathrm{e}-09 \backslash \mathrm{RMSF}=1.905 \mathrm{e}-06 \backslash \mathrm{Dipole}$ $=2.7230519,-1.0052687,0.3665722 \backslash \mathrm{PG}=\mathrm{C} 01[\mathrm{X}(\mathrm{C} 11 \mathrm{H} 11 \mathrm{~N} 1 \mathrm{O} 2)] \backslash \backslash @$

\section{DHP/PBQ TS1 (UB3-LYP, heptane)}

$1 \backslash 1 \backslash G I N C-A C 17 \backslash F T S \backslash U B 3 L Y P \backslash 6-31+G(d, p) \backslash C 11 H 11 N 102 \backslash B U C 562 \backslash 31-J a n-2006 \backslash 0 \backslash \backslash$ \# UB3LYP $/ 6-31+G(D, P)$ GUESS $=(M I X, A L W A Y S)$ OPT $=(T S, C A L C F C$, NOE IGENTEST $)$ SC $\mathrm{RF}=($ READ , IEFPCM, $\mathrm{SOLVENT}=\mathrm{HEPTANE}) \backslash \backslash \mathrm{OC} 6 \mathrm{H} 4 \mathrm{O}$ TS C $5 \mathrm{H} 6 \mathrm{NH}$ B3Opt_Heptane_02 $\backslash \backslash 0$ $, 1 \backslash C,-0.5906760765,1.2086792292,-0.50669 \overline{1} 33 \overline{9} 9 \backslash C_{,}-1.44827 \overline{2} 7785,1 . \overline{4} 24910$ $8312,0.6310381705 \backslash \mathrm{C},-2.5269445663,0.6212039329,0.8688211535 \backslash \mathrm{C},-2.83498$ $16672,-0.5199034675,0.0076354367 \backslash \mathrm{C},-1.9517151109,-0.7162089649,-1.1459$ $018463 \backslash \mathrm{C},-0.8888689265,0.1070422909,-1.3830148918 \backslash \mathrm{H},-1.2204707353,2.27$ $15246408,1.274044929 \backslash \mathrm{H},-3.1896245933,0.7930862688,1.7121838753 \backslash \mathrm{H},-2.19$ $42649519,-1.5395858498,-1.8120668397 \backslash \mathrm{H},-0.245340162,-0.0318117917,-2.2$ $481095322 \backslash 0,0.4051952659,2.0143392907,-0.7636904955 \backslash 0,-3.7961528157,-1$ $.2871249996,0.237861579 \backslash \mathrm{H}, 1.4700043069,1.6050147867,-0.3206633988 \backslash \mathrm{C}, 2$. $4594480175,0.9564088131,0.3238627612 \backslash \mathrm{C}, 1.7066331268,0.3113242097,1.393$ $3275379 \backslash \mathrm{C}, 1.3774992281,-1.0101266669,1.3232937566 \backslash \mathrm{N}, 1.8416352368,-1.79$ $77948471,0.2999268252 \backslash \mathrm{C}, 2.6818888389,-1.2803609533,-0.6684700587 \backslash \mathrm{C}, 3.0$ $318561585,0.03347534,-0.6517054047 \backslash \mathrm{H}, 3.0624519024,1.8267222106,0.59463$ $69251 \backslash \mathrm{H}, 1.3530733213,0.889749103,2.2391534209 \backslash \mathrm{H}, 0.7636144744,-1.507037$ $1614,2.0651184047 \backslash \mathrm{H}, 1.5794976502,-2.7736380079,0.2646539251 \backslash \mathrm{H}, 3.049227$ $4017,-1.9866932496,-1.403104279 \backslash \mathrm{H}, 3.7128476628,0.4008510855,-1.4118755$ $237 \backslash \backslash$ Version=IA64L-G03RevC.02 $\backslash$ State $=1-A \backslash H F=-630.9446488 \backslash \mathrm{S} 2=0.128487 \backslash \mathrm{S} 2$ $-1=0 . \backslash \mathrm{S} 2 \mathrm{~A}=0.001848 \backslash \mathrm{RMSD}=5.331 \mathrm{e}-09 \backslash \mathrm{RMSF}=3.486 \mathrm{e}-06 \backslash \mathrm{Dipole}=3.5429144,-0.8$ $790793,0.3816787 \backslash \mathrm{PG}=\mathrm{C} 01 \quad[\mathrm{X}(\mathrm{C} 11 \mathrm{H} 11 \mathrm{~N} 1 \mathrm{O} 2)] \backslash \backslash @$ 


\section{DHP/PBQ TS1 (UB3-LYP, ether)}

$1 \backslash 1 \backslash G I N C-A C 37 \backslash F T S \backslash U B 3 L Y P \backslash 6-31+G(d, p) \backslash C 11 \mathrm{H} 11 \mathrm{~N} 102 \backslash B U C 562 \backslash 31-J a n-2006 \backslash 0 \backslash \backslash$ \# UB3LYP $/ 6-31+G(D, P)$ GUESS $=(M I X, A L W A Y S)$ OPT=(TS, CALCFC, NOE IGENTEST $)$ SC

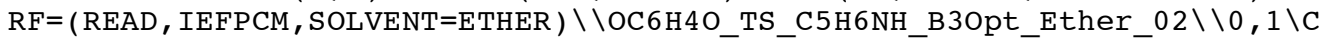
$,-0.5978912771,1.1922897517,-0.5107897 \overline{7} 16 \backslash \bar{C},-1.4 \overline{5} 80004 \overline{9} 92,1 . \overline{4} 14576314$, $0.6252258796 \backslash \mathrm{C},-2.5418966542,0.6167298906,0.8625675766 \backslash \mathrm{C},-2.8534857458$ $,-0.523329366,0.0054973627 \backslash C,-1.9612409504,-0.7386376193,-1.1348548418$ $\backslash \mathrm{C},-0.8912324069,0.0755460648,-1.3718890455 \backslash \mathrm{H},-1.2314230444,2.26372503$ $26,1.2660870588 \backslash \mathrm{H},-3.2048971378,0.7984842763,1.704343495 \backslash \mathrm{H},-2.19762585$ $39,-1.5699074438,-1.7942665178 \backslash \mathrm{H},-0.2416293399,-0.0799801676,-2.230114$ $6098 \backslash 0,0.3813128928,2.0068736266,-0.7860214497 \backslash 0,-3.8312863532,-1.2789$ $944345,0.2295202596 \backslash \mathrm{H}, 1.482262537,1.6100684492,-0.3027308231 \backslash \mathrm{C}, 2.41949$ $42346,0.9828924814,0.362846396 \backslash \mathrm{C}, 1.6278617782,0.291003823,1.3757719957$ $\backslash \mathrm{C}, 1.3649304304,-1.0457006543,1.2701920334 \backslash \mathrm{N}, 1.9210426734,-1.794037393$ $3,0.2702325734 \backslash \mathrm{C}, 2.7892459254,-1.2301862116,-0.6433473432 \backslash \mathrm{C}, 3.08203448$ $14,0.0972529524,-0.5928144881 \backslash \mathrm{H}, 2.9872708679,1.8587402277,0.6941515961$ $\backslash \mathrm{H}, 1.2083895961,0.8386081835,2.2121940671 \backslash \mathrm{H}, 0.7374417814,-1.5822548217$ $, 1.9732858896 \backslash \mathrm{H}, 1.7167523343,-2.7878980133,0.2176362065 \backslash \mathrm{H}, 3.2277177417$ $,-1.9126869919,-1.3622878754 \backslash \mathrm{H}, 3.7893135894,0.5037049264,-1.3083515027$ $\backslash \backslash$ Version $=I A 64 \mathrm{~L}-\mathrm{G} 03$ RevC $.02 \backslash$ State $=1-\mathrm{A} \backslash \mathrm{HF}=-630.9554784 \backslash \mathrm{S} 2=0 . \backslash \mathrm{S} 2-1=0 . \backslash \mathrm{S} 2 \mathrm{~A}$ $=0 . \backslash \mathrm{RMSD}=4.075 \mathrm{e}-09 \backslash \mathrm{RMSF}=3.351 \mathrm{e}-06 \backslash \mathrm{Dipole}=4.30373,-1.0103461,0.4649217 \backslash$ $\mathrm{PG}=\mathrm{C} 01[\mathrm{X}(\mathrm{C} 11 \mathrm{H} 11 \mathrm{~N} 1 \mathrm{O} 2)] \backslash \backslash @$

\section{DHP/PBQ TS1 (UB3-LYP, acetone)}

$1 \backslash 1 \backslash G I N C-A C 32 \backslash F T S \backslash U B 3 L Y P \backslash 6-31+G(d, p) \backslash C 11 \mathrm{H} 11 \mathrm{~N} 102 \backslash B U C 562 \backslash 31-J a n-2006 \backslash 0 \backslash \backslash$ \# UB3LYP/6-31+G (D,P) GUESS=(MIX, ALWAYS ) OPT=(TS, CALCFC, NOE IGENTEST) SC $\mathrm{RF}=(\mathrm{READ}, \mathrm{IEFPCM}, \mathrm{SOLVENT}=\mathrm{ACETONE}) \backslash \backslash \mathrm{OC} 6 \mathrm{H} 4 \mathrm{O} \_\mathrm{TS}$ C5H6NH_B3Opt_Acetone_02\\0 $, 1 \backslash C,-0.6428170474,1.1449647272,-0.52753 \overline{5} 75 \overline{5} 1 \backslash \mathrm{C},-1.52204 \overline{2} 0531,1 . \overline{4} 09155$ $8767,0.5849461135 \backslash \mathrm{C},-2.6113820985,0.621945504,0.8328570596 \backslash \mathrm{C},-2.915531$ $166,-0.5440146442,0.0093565155 \backslash \mathrm{C},-1.9987828821,-0.8100336044,-1.098282$ $9133 \backslash \mathrm{C},-0.9178911448,-0.0120700127,-1.3422845164 \backslash \mathrm{H},-1.3053956526,2.280$ $1833502,1.199060939 \backslash \mathrm{H},-3.286067322,0.835774066,1.6574837359 \backslash \mathrm{H},-2.21863$ $12088,-1.6698870419,-1.7256452908 \backslash \mathrm{H},-0.2479477944,-0.2096346467,-2.175$ $703842 \backslash 0,0.3262408437,1.9595899943,-0.8281050698 \backslash 0,-3.9089480132,-1.28$ $06650165,0.2374588603 \backslash \mathrm{H}, 1.4427872138,1.6080712936,-0.2886795304 \backslash \mathrm{C}, 2.35$ $91728726,1.0396557531,0.4239818128 \backslash \mathrm{C}, 1.5507098399,0.2512953684,1.35174$ $99061 \backslash \mathrm{C}, 1.4140681845,-1.0993912952,1.188050704 \backslash \mathrm{N}, 2.1103703464,-1.76214$ $24023,0.2181784279 \backslash \mathrm{C}, 3.0051013033,-1.1012428428,-0.5996526566 \backslash \mathrm{C}, 3.1774$ $06328,0.2430288313,-0.4908239017 \backslash \mathrm{H}, 2.8270470105,1.9422741503,0.8305035$ $212 \backslash \mathrm{H}, 1.0292939279,0.731195074,2.1716496764 \backslash \mathrm{H}, 0.7852746041,-1.71193723$ $31,1.82434287 \backslash \mathrm{H}, 2.0050122284,-2.768725587,0.1292387983 \backslash \mathrm{H}, 3.5613847401$, $-1.7234884616,-1.2907503411 \backslash \mathrm{H}, 3.9082343661,0.7300100617,-1.1277540659 \backslash$ $\backslash$ Version $=I A 64 \mathrm{~L}-\mathrm{G} 03 \mathrm{RevC} .02 \backslash \mathrm{State}=1-\mathrm{A} \backslash \mathrm{HF}=-630.9576068 \backslash \mathrm{S} 2=0 . \backslash \mathrm{S} 2-1=0 . \backslash \mathrm{S} 2 \mathrm{~A}=$ $0 . \backslash \mathrm{RMSD}=9.191 \mathrm{e}-09 \backslash \mathrm{RMSF}=4.710 \mathrm{e}-06 \backslash \mathrm{Dipole}=4.7496148,-1.046006,0.4990261 \backslash$ $\mathrm{PG}=\mathrm{C} 01[\mathrm{X}(\mathrm{C} 11 \mathrm{H} 11 \mathrm{~N} 102)] \backslash \backslash @$ 


\section{DHP/PBQ TS1 (UB3-LYP, $\mathrm{CH}_{3} \mathrm{CN}$ )}

$1 \backslash 1 \backslash G I N C-A C 22 \backslash F T S \backslash U B 3 L Y P \backslash 6-31+G(d, p) \backslash C 11 \mathrm{H} 11 \mathrm{~N} 102 \backslash B U C 562 \backslash 08-J u n-2006 \backslash 0 \backslash \backslash$ \# UB3LYP/6-31+G(d,p) Guess=(Mix,Always) Opt=(TS,CalcFC,NoEigenTest) SC

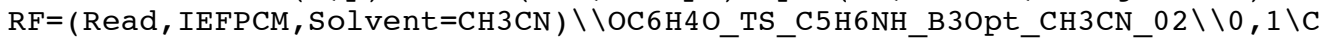
$,-0.6265429225,1.1676951022,-0.5255014011 \backslash \mathrm{C},-1.4 \overline{7} 80341 \overline{4} 54,1 . \overline{4} 637491041$ $, 0.6006250932 \backslash \mathrm{C},-2.5860816973,0.710839306,0.8715673625 \backslash \mathrm{C},-2.9390431275$ $,-0.4495412098,0.0602219904 \backslash C,-2.0491994253,-0.7500112154,-1.060126685$ $8 \backslash \mathrm{C},-0.9493401584,0.0138844478,-1.3274659317 \backslash \mathrm{H},-1.2247620827,2.3308359$ $851,1.2062347644 \backslash \mathrm{H},-3.2394627398,0.9492685056,1.7065780055 \backslash \mathrm{H},-2.304342$ $3169,-1.6071867535,-1.6777436537 \backslash \mathrm{H},-0.2995387839,-0.2091963828,-2.1703$ $450309 \backslash 0,0.3597422916,1.9518444605,-0.8484123943 \backslash 0,-3.9512225305,-1.15$ $41321105,0.3087709816 \backslash \mathrm{H}, 1.4792273194,1.5684807318,-0.3232601882 \backslash \mathrm{C}, 2.38$ $52557962,0.9776998537,0.3773062104 \backslash \mathrm{C}, 1.5680298758,0.2196436644,1.32295$ $42424 \backslash \mathrm{C}, 1.390724605,-1.1279733467,1.1720766962 \backslash \mathrm{N}, 2.0530943674,-1.81744$ $57779,0.1974735816 \backslash \mathrm{C}, 2.9535600366,-1.1889134448,-0.6390483804 \backslash \mathrm{C}, 3.1660$ $615213,0.1506386179,-0.5436876156 \backslash \mathrm{H}, 2.8874022104,1.8673114919,0.771885$ $185 \backslash \mathrm{H}, 1.0732090398,0.7205087708,2.1468230821 \backslash \mathrm{H}, 0.7545269242,-1.7176542$ $281,1.8225370888 \backslash \mathrm{H}, 1.9181811076,-2.8216544566,0.1185095804 \backslash \mathrm{H}, 3.4811407$ $967,-1.8323188889,-1.3333206112 \backslash \mathrm{H}, 3.9008630385,0.6114827733,-1.1953559$ $715 \backslash \backslash$ Version=IA64L-G03RevD. $01 \backslash$ State $=1-A \backslash H F=-630.9584448 \backslash \mathrm{S} 2=0 . \backslash \mathrm{S} 2-1=0 . \backslash$ $\mathrm{S} 2 \mathrm{~A}=0 . \backslash \mathrm{RMSD}=4.095 \mathrm{e}-09 \backslash \mathrm{RMSF}=3.758 \mathrm{e}-06 \backslash \mathrm{Thermal}=0 . \backslash \mathrm{Dipole}=4.7783743,-1.20$ $3306,0.4397187 \backslash \mathrm{PG}=\mathrm{C} 01 \quad[\mathrm{X}(\mathrm{C} 11 \mathrm{H} 11 \mathrm{~N} 1 \mathrm{O} 2)] \backslash \backslash @$ 
TABLE S2: MPWB1K/6-311+G(3df,2p) Total Energies for Equilibrium Structures (0 K, hartrees)

\begin{tabular}{|c|c|c|c|c|c|}
\hline & Substrate & $\mathrm{DH}_{2}$ & $\mathrm{DH} \bullet$ & $\mathrm{DH}+$ & $\mathrm{D}$ \\
\hline \multirow[t]{5}{*}{ Donors $\left(\mathrm{DH}_{2}\right)$} & ACD & -556.45746 & -555.84955 & -555.64598 & -555.26974 \\
\hline & CHD & -233.23507 & -232.62279 & -232.37702 & -232.09041 \\
\hline & DHA & -540.39455 & -539.77928 & -539.55107 & -539.21501 \\
\hline & DHP & -249.29350 & -248.68884 & -248.49638 & -248.14100 \\
\hline & & $\mathrm{A}$ & $\mathrm{AH} \bullet$ & $\mathrm{AH}-$ & $\mathrm{AH}_{2}$ \\
\hline \multirow[t]{3}{*}{ Acceptors (A) } & AMS & -348.71303 & -349.28454 & -349.30490 & -349.91360 \\
\hline & DDQ & -1485.12053 & -1485.72630 & -1485.85733 & -1486.36129 \\
\hline & PBQ & -381.30923 & -381.90327 & -381.96751 & -382.52952 \\
\hline Radical trap & $\mathrm{PhNO}$ & -361.37438 & & & \\
\hline Adducts & {$[\mathrm{DH}-\mathrm{PhNO}]_{\bullet}$} & -917.26024 & & & \\
\hline$\left(\mathrm{DH}_{2}=\mathrm{ACD}\right)$ & {$[\mathrm{DH}-\mathrm{PhNO}]^{+}$} & -917.01240 & & & \\
\hline
\end{tabular}

TABLE S3: MPWB1K/6-311+G(3df,2p) Total Energies for Transition Structures (0 K, hartrees)

\begin{tabular}{|c|c|c|c|c|c|}
\hline \multirow[t]{2}{*}{ Geometry } & & \multicolumn{4}{|c|}{ Reaction } \\
\hline & & 1 & 2 & 5 & 6 \\
\hline \multirow[t]{2}{*}{ Gas phase } & TS1 (R) & -905.11519 & -1718.33445 & -614.50905 & -630.57663 \\
\hline & TS1 (U) & -905.12034 & -1718.33793 & -614.51286 & -630.58107 \\
\hline Heptane & TS1 (U) & -905.12026 & -1718.33712 & -614.51261 & -630.57968 \\
\hline Ether & TS1 (U) & -905.12018 & -1718.33650 & -614.51255 & -630.57723 \\
\hline Acetone & TS1 (U) & -905.12005 & -1718.33632 & -614.51212 & -630.57746 \\
\hline \multirow[t]{2}{*}{$\mathrm{CH}_{3} \mathrm{CN}$} & TS1 (U) & -905.12003 & -1718.33628 & -614.51203 & -630.57742 \\
\hline & & 1 & & & \\
\hline \multirow[t]{2}{*}{ Gas phase } & $\mathrm{TS} 2(\mathrm{R})$ & -905.10633 & & \multicolumn{2}{|c|}{$\mathrm{DH}_{2}=\mathrm{ACD}$} \\
\hline & TS2 (U) & -905.11824 & & \multicolumn{2}{|c|}{$\mathrm{DH} \bullet+\mathrm{PhNO} \rightarrow[\mathrm{DH}-\mathrm{PhNH}] \bullet$} \\
\hline Heptane & $\mathrm{TS} 2(\mathrm{U})$ & -905.11821 & & \multicolumn{2}{|c|}{-917.21689} \\
\hline Ether & TS2 (U) & -905.11830 & & \multicolumn{2}{|c|}{$\mathrm{DH}^{+}+\mathrm{PhNO} \rightarrow[\mathrm{DH}-\mathrm{PhNH}]^{+}$} \\
\hline Acetone & TS2 (U) & -905.11822 & & \multicolumn{2}{|c|}{-917.01216} \\
\hline $\mathrm{CH}_{3} \mathrm{CN}$ & TS2 (U) & -905.11822 & & & \\
\hline
\end{tabular}


TABLE S4: Condensed-Phase UMPWB1K/6-311+G(3df,2p) Free-Energy Profiles ${ }^{\mathrm{a}}\left(\mathrm{kJ} \mathrm{mol}^{-1}\right)$ for the Transfer-Hydrogenation Reaction Between Acridan and $\alpha$-Methylstyrene, with Free Energy of Solvation Obtained with Various Methodologies

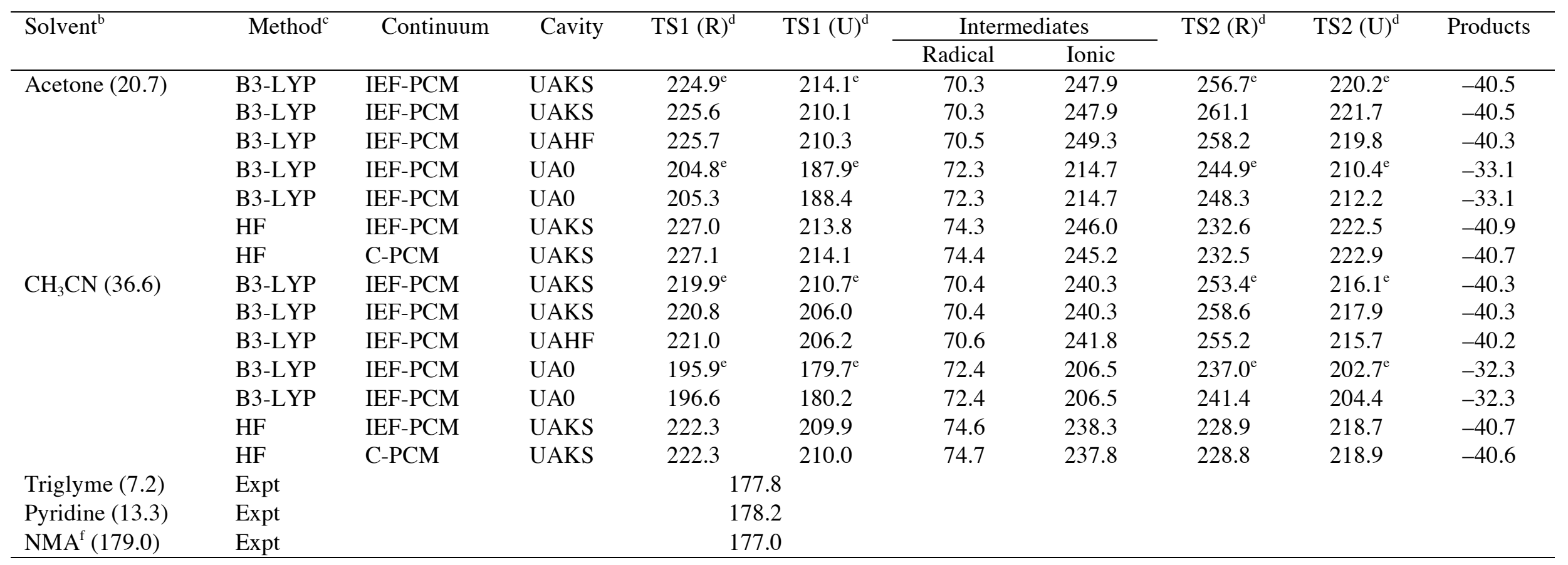

${ }^{\text {a }} 573 \mathrm{~K}$ values. ${ }^{\text {b }}$ Dielectric constants $(\varepsilon)$ of the solvents are shown in parentheses. ${ }^{c}$ The $6-31+\mathrm{G}(\mathrm{d}, \mathrm{p})$ basis set was used in the calculation of $\Delta \mathrm{G}_{\text {solv }}$. ${ }^{\mathrm{d}}$

Condensed-phase geometries were used unless noted otherwise. ${ }^{\mathrm{e}}$ Gas-phase geometries. ${ }^{\mathrm{f}} \mathrm{N}$-methylacetamide. 
TABLE S5: Free Energies of Solvation $\left(\mathrm{kcal} \mathrm{mol}^{-1}, 573 \mathrm{~K}, 1 \mathrm{~mol} \mathrm{~L}^{-1}\right.$ ) for the ACD/AMS Reaction, Calculated with Various Methods ${ }^{\mathrm{a}}$

\begin{tabular}{|c|c|c|c|c|c|c|c|c|c|c|c|c|}
\hline & \multicolumn{4}{|c|}{$\begin{array}{c}\text { B3-LYP/IEF-PCM/UAKS// } \\
\text { Gas-phase geometry }\end{array}$} & \multicolumn{4}{|c|}{$\begin{array}{l}\text { B3-LYP/IEF-PCM/UAKS// } \\
\text { Condensed-phase geometry }\end{array}$} & \multicolumn{4}{|c|}{$\begin{array}{l}\text { B3-LYP/IEF-PCM/UAHF// } \\
\text { Condensed-phase geometry }\end{array}$} \\
\hline & Heptane & Ether & Acetone & $\mathrm{CH}_{3} \mathrm{CN}$ & Heptane & Ether & Acetone & $\mathrm{CH}_{3} \mathrm{CN}$ & Heptane & Ether & Acetone & $\mathrm{CH}_{3} \mathrm{CN}$ \\
\hline $\mathrm{ACD}\left(\mathrm{DH}_{2}\right)$ & & & & & 10.13 & 14.13 & 11.14 & 19.38 & 10.22 & 14.41 & 11.40 & 19.67 \\
\hline $\mathrm{DH} \bullet$ & & & & & 10.31 & 14.29 & 11.15 & 19.37 & 10.41 & 14.60 & 11.45 & 19.70 \\
\hline $\mathrm{DH}^{+}$ & & & & & -7.30 & -15.57 & -24.02 & -16.61 & -7.11 & -15.03 & -23.41 & -15.96 \\
\hline $\mathrm{D}$ & & & & & 10.36 & 14.71 & 11.53 & 19.69 & 10.50 & 14.92 & 11.83 & 20.01 \\
\hline $\operatorname{AMS}(\mathrm{A})$ & & & & & 9.61 & 13.86 & 11.50 & 18.20 & 9.61 & 13.86 & 11.50 & 18.20 \\
\hline $\mathrm{AH} \bullet$ & & & & & 8.91 & 13.18 & 10.82 & 17.56 & 8.91 & 13.18 & 10.82 & 17.56 \\
\hline $\mathrm{AH}^{-}$ & & & & & -10.69 & -19.69 & -28.98 & -23.27 & -10.69 & -19.69 & -28.98 & -23.27 \\
\hline $\mathrm{AH}_{2}$ & & & & & 9.10 & 13.48 & 11.11 & 17.93 & 9.10 & 13.48 & 11.11 & 17.93 \\
\hline TS1 (R) & 22.25 & 29.99 & 24.80 & 38.54 & 22.27 & 30.03 & 24.81 & 38.56 & 22.35 & 30.36 & 25.11 & 38.91 \\
\hline TS1 (U) & 24.13 & 32.20 & 26.82 & 40.97 & 23.04 & 30.95 & 25.65 & 39.59 & 23.12 & 31.28 & 25.96 & 39.93 \\
\hline TS2 (R) & 24.26 & 33.26 & 27.95 & 42.10 & 24.39 & 33.64 & 28.42 & 42.68 & 24.04 & 33.16 & 27.99 & 42.14 \\
\hline \multirow[t]{3}{*}{ TS2 (U) } & 23.56 & 32.47 & 27.23 & 41.19 & 23.55 & 32.44 & 27.23 & 41.21 & 23.35 & 32.22 & 27.04 & 40.96 \\
\hline & \multicolumn{4}{|c|}{$\begin{array}{l}\text { B3-LYP/IEF-PCM/UA0// } \\
\text { Gas-phase geometry }\end{array}$} & \multicolumn{4}{|c|}{$\begin{array}{l}\text { B3-LYP/IEF-PCM/UA0// } \\
\text { Condensed-phase geometry }\end{array}$} & & & & \\
\hline & Heptane & Ether & Acetone & $\mathrm{CH} 3 \mathrm{CN}$ & Heptane & Ether & Acetone & $\mathrm{CH}_{3} \mathrm{CN}$ & & & & \\
\hline $\mathrm{ACD}\left(\mathrm{DH}_{2}\right)$ & & & & & 12.37 & 15.98 & 9.95 & 18.43 & & & & \\
\hline $\mathrm{DH} \bullet$ & & & & & 11.92 & 15.13 & 8.82 & 17.16 & & & & \\
\hline $\mathrm{DH}^{+}$ & & & & & -8.88 & -18.19 & -32.41 & -25.02 & & & & \\
\hline $\mathrm{D}$ & & & & & 12.61 & 16.43 & 10.84 & 19.22 & & & & \\
\hline $\operatorname{AMS}(\mathrm{A})$ & & & & & 13.68 & 18.01 & 14.26 & 21.43 & & & & \\
\hline $\mathrm{AH} \bullet$ & & & & & 14.24 & 18.85 & 15.19 & 22.54 & & & & \\
\hline $\mathrm{AH}^{-}$ & & & & & -6.01 & -14.43 & -26.94 & -20.66 & & & & \\
\hline $\mathrm{AH}_{2}$ & & & & & 14.33 & 18.93 & 15.15 & 22.60 & & & & \\
\hline TS1 (R) & 23.87 & 30.47 & 21.55 & 35.08 & 23.88 & 30.45 & 21.54 & 35.06 & & & & \\
\hline TS1 (U) & 24.47 & 31.23 & 22.14 & 35.84 & 24.45 & 31.13 & 22.03 & 35.72 & & & & \\
\hline $\mathrm{TS} 2(\mathrm{R})$ & 26.55 & 34.49 & 26.71 & 40.46 & 26.61 & 34.62 & 26.93 & 40.83 & & & & \\
\hline TS2 (U) & 26.36 & 34.30 & 26.47 & 40.27 & 26.36 & 34.32 & 26.52 & 40.25 & & & & \\
\hline
\end{tabular}

${ }^{\mathrm{c}}$ The $6-31+\mathrm{G}(\mathrm{d}, \mathrm{p})$ basis set was used in the calculation of $\Delta \mathrm{G}_{\mathrm{solv}}$. 
TABLE S5: Contd

\begin{tabular}{lcccccccc}
\hline & \multicolumn{3}{c}{$\begin{array}{c}\text { HF/IEF-PCM/UAKS// } \\
\text { Condensed-phase geometry }\end{array}$} & \multicolumn{3}{c}{$\begin{array}{c}\text { HF/C-PCM/UAKS // } \\
\text { Condensed-phase geometry }\end{array}$} \\
\cline { 2 - 9 } & Heptane & Ether & Acetone & $\mathrm{CH}_{3} \mathrm{CN}$ & Heptane & Ether & Acetone & $\mathrm{CH}_{3} \mathrm{CN}$ \\
\hline $\mathrm{ACD}\left(\mathrm{DH}_{2}\right)$ & 9.89 & 13.5 & 10.42 & 18.62 & 9.20 & 12.58 & 10.21 & 18.50 \\
$\mathrm{DH} \bullet$ & 10.28 & 14.21 & 11.06 & 19.28 & 9.64 & 13.37 & 10.87 & 19.16 \\
$\mathrm{DH}^{+}$ & -7.46 & -15.99 & -24.45 & -17.07 & -8.20 & -16.92 & -24.67 & -17.19 \\
$\mathrm{D}$ & 10.07 & 13.94 & 10.67 & 18.79 & 9.51 & 13.25 & 10.50 & 18.69 \\
$\mathrm{AMS}(\mathrm{A})$ & 9.49 & 13.52 & 11.12 & 17.8 & 9.24 & 13.20 & 11.05 & 17.76 \\
$\mathrm{AH} \bullet$ & 8.9 & 13.13 & 10.77 & 17.5 & 8.71 & 12.88 & 10.71 & 17.47 \\
$\mathrm{AH}{ }^{-}$ & -11.11 & -20.72 & -30.09 & -24.43 & -11.95 & -21.69 & -30.34 & -24.59 \\
$\mathrm{AH}{ }_{2}$ & 8.99 & 13.18 & 10.78 & 17.58 & 8.77 & 12.88 & 10.71 & 17.54 \\
$\mathrm{TS}$ 1 (R) & 22.02 & 29.34 & 24.06 & 37.77 & 21.20 & 28.22 & 23.80 & 37.61 \\
$\mathrm{TS}$ 1 (U) & 22.97 & 30.77 & 25.45 & 39.38 & 22.24 & 29.76 & 25.23 & 39.24 \\
TS2 (R) & 21.87 & 27.43 & 20.52 & 34.41 & 20.90 & 26.18 & 20.20 & 34.22 \\
TS2 (U) & 23.27 & 31.68 & 26.32 & 40.24 & 22.69 & 30.91 & 26.13 & 40.13 \\
\hline
\end{tabular}


TABLE S6: Free Energies of Solvation $\left(\mathrm{kcal} \mathrm{mol}^{-1}, 298 \mathrm{~K}, 1 \mathrm{~mol} \mathrm{~L}^{-1}\right)$ for the CHD/DDQ Reaction, Calculated with Various Methods ${ }^{\mathrm{a}}$

\begin{tabular}{lcccccccc}
\hline & \multicolumn{3}{c}{ B3-LYP/IEF-PCM/UAKS } & \multicolumn{3}{c}{ B3-LYP/IEF-PCM/UA0 } \\
\cline { 2 - 9 } & Heptane & Ether & Acetone & $\mathrm{CH}_{3} \mathrm{CN}$ & Heptane & Ether & Acetone & $\mathrm{CH}_{3} \mathrm{CN}$ \\
\hline CHD $\left(\mathrm{DH}_{2}\right)$ & -2.85 & -1.68 & -2.25 & 0.53 & -0.51 & 0.94 & -0.72 & 2.36 \\
DH• & -2.57 & -1.43 & -2.08 & 0.69 & -0.74 & 0.44 & -1.37 & 1.59 \\
DH- & -25.25 & -40.25 & -47.20 & -45.44 & -26.52 & -40.76 & -52.18 & -50.33 \\
D & -1.94 & -0.57 & -1.35 & 1.47 & -1.00 & -0.08 & -2.04 & 0.8 \\
DDQ (A) & -1.69 & -1.28 & -3.89 & 1.11 & -1.46 & -1.84 & -7.66 & -2.96 \\
AH• & -2.85 & -3.25 & -6.15 & -1.17 & -3.04 & -4.53 & -11.32 & -6.75 \\
AH- & -20.98 & -33.12 & -42.76 & -38.72 & -20.34 & -32.01 & -44.87 & -40.93 \\
AH2 & -2.66 & -1.28 & -4.78 & 0.31 & -2.77 & -3.65 & -9.90 & -5.25 \\
TS1 (R) & -1.83 & -2.13 & -5.73 & 1.04 & -2.29 & -3.33 & -11.11 & -4.79 \\
TS1 (U) & -1.74 & -2.10 & -5.61 & 1.19 & -2.21 & -3.22 & -11.04 & -4.66 \\
TS1 (R) & -1.70 & -1.63 & -5.00 & 1.91 & -2.13 & -2.66 & -10.03 & -3.58 \\
TS1 (U) & -1.70 & -1.63 & -5.00 & 1.91 & -2.13 & -2.66 & -10.03 & -3.58 \\
\hline
\end{tabular}

${ }^{\mathrm{a}}$ The $6-31+\mathrm{G}(\mathrm{d}, \mathrm{p})$ basis set was used in the calculation of $\Delta \mathrm{G}_{\mathrm{solv}} \cdot{ }^{\mathrm{b}}$ Gas-phase geometries. Condensed-phase geometries. 
Table S7: Free Energies of Solvation $\left(\mathrm{kcal} \mathrm{mol}^{-1}, 298 \mathrm{~K}, 1 \mathrm{~mol} \mathrm{~L}^{-1}\right.$ ) for the CHD/PBQ and DHP/PBQ Reactions, Calculated with the B3-LYP/6-31+G(d,p)/IEF-PCM/UA0 ${ }^{a}$

\begin{tabular}{|c|c|c|c|c|}
\hline & Heptane & Ether & Acetone & $\mathrm{CH}_{3} \mathrm{CN}$ \\
\hline $\mathrm{CHD}\left(\mathrm{DH}_{2}\right)$ & -0.51 & 0.94 & -0.72 & 2.36 \\
\hline $\mathrm{DH} \bullet$ & -0.74 & 0.44 & -1.37 & 1.59 \\
\hline $\mathrm{DH}^{+}$ & -26.52 & -40.76 & -52.18 & -50.33 \\
\hline D & -1.00 & -0.08 & -2.04 & 0.8 \\
\hline $\operatorname{DHP}\left(\mathrm{D}^{\prime} \mathrm{H}_{2}\right)$ & -2.17 & -2.15 & -4.99 & -2.21 \\
\hline $\mathrm{D}^{\prime} \mathrm{H} \bullet$ & -2.59 & -3.02 & -6.20 & -3.55 \\
\hline $\mathrm{D}^{\prime} \mathrm{H}^{+}$ & -29.19 & -45.53 & -58.55 & -57.04 \\
\hline $\mathrm{D}^{\prime}$ & -1.74 & -1.65 & -4.26 & -1.61 \\
\hline PBQ (A) & -2.44 & -3.07 & -6.65 & -3.59 \\
\hline $\mathrm{AH} \bullet$ & -4.56 & -7.18 & -12.82 & -10.00 \\
\hline $\mathrm{AH}^{-}$ & -27.90 & -45.32 & -60.74 & -59.08 \\
\hline $\mathrm{AH}_{2}$ & -4.56 & -7.02 & -12.72 & -9.85 \\
\hline $\mathrm{TS} 1_{\mathrm{CHD}}(\mathrm{U})$ & -3.49 & -4.42 & -10.17 & -5.35 \\
\hline $\mathrm{TS}_{\mathrm{DHP}}(\mathrm{U})$ & -6.71 & -10.67 & -19.21 & -14.62 \\
\hline \multicolumn{2}{|c|}{$\mathrm{D} " \mathrm{H} \bullet+\mathrm{PhNO} \rightarrow[\mathrm{D} " \mathrm{H}-\mathrm{PhNH}] \bullet \mathrm{TS}$} & \multirow{4}{*}{$\left(D^{\prime \prime} H_{2}=A C D\right)$} & & -5.19 \\
\hline \multicolumn{2}{|c|}{$\mathrm{D}^{\prime \prime} \mathrm{H}^{+}+\mathrm{PhNO} \rightarrow[\mathrm{D} " \mathrm{H}-\mathrm{PhNH}]^{+} \mathrm{TS}$} & & & -36.79 \\
\hline \multicolumn{2}{|c|}{ [D"H-PhNO]• } & & & -2.83 \\
\hline \multicolumn{2}{|c|}{$[\mathrm{D} " \mathrm{H}-\mathrm{PhNO}]^{+}$} & & & -36.70 \\
\hline
\end{tabular}

